(112.8 pm for $\mathrm{CO}$ ). This will not be the case in more complicated molecules as you will see below. For $\mathrm{CO}$, it is clear that the energy will become zero at $r_{0}$, i.e. the molecule is free of 'strain'. Again this will be different in more complicated cases.

Now let us see, how we could extend our mechanical model to also predict the structure of $\mathrm{CO}_{2}$, which has longer bond-lengths $(116.0 \mathrm{pm})$. We could assume that there is a repulsive force between atoms which are not directly bound. We could e.g. use the following assumption for this part of the potential:

$\mathrm{E}_{\mathrm{r}}=\mathrm{c}_{2} \mathrm{e}^{-\mathrm{r}^{\prime}}$

$r^{\prime}$ is the distance between nonbonded atoms in our case between the two oxygens. $c_{2}$ is again an empirical parameter, which is stored in the program. Now the total energy for this molecule reads as:

$E=2 E_{s}+E_{r}=2 c_{1}\left(r-r_{0}\right)^{2}+c_{2} e^{-r}$

Again the program can calculate $E$, having $r$ and $r^{\prime}$ from the input data and it will find an energy minimum by systematically changing the distances. This time the final distance will be slightly longer due to the last term and the energy will not be exactly zero, which means that there will be some 'strain' in this molecule. 'Strain in $\mathrm{CO}_{2}$ ' you might ask. Yes, but from the point of view of this force field, not in the usual chemical sense (the above force field is any- way not a very realistic one, but rather a simplified model designed to show the basic ideas). Such a simple example as this could easily be handled with paper and pencil, but medium sized molecules already need the help of a computer.

Now, what do we mean by a force field? The force field is generated from a set of assumptions using a necessary number of parameters. As you can imagine, the force field is the critical part of a molecular mechanics model. It depends on the force field how well experimental structures or enthalpies are modeled, and also how meaningful in the chemical sense, things like strain can be interpreted. Therefore, we will discuss in a later column aspects of force fields in more details.

Perhaps you are now eager to get experience on your personal computer. This would surely be the best way to get acquainted with molecular modeling. There are several good programs available which implement these two concepts, and you will find reviews about such programs in our column. Now, are you on your way?

\section{Announcements}

Workshop on Molecular Mechanics and Molecular Dynamics Simulations

Lausanne, October 2nd and 3rd, 1990

Organized by the Group of Swiss Computationa Chemists (GSCC). Participants limited to 30 . Thi

workshop is intended to have a triple purpose: i) to present the basic features of the physical principles and mathematical formalism of these theoretical models ii) to revicw their most important applications to chemistry and biology; iii) to enable the participants to have a first start in the practice of these techniques by performing some guided calculations. Lectures by $\mathrm{H}$ J.C. Berendsen, Univ of Groningen, M. Dobler. ETH Zürich, and S. Wodak, Free Univ, of Brussels. For further information contact: Prof. J. Weber, Dept. of Physical Chemistry, University of Geneva, 30 , quai Ernest Ansermet, $\mathrm{CH}-1211$ Geneva 4 (Tel. 022/7026530; EM: WEBER@SC2A.UNIGE.CH Fax: 022296102 ).

VIIth International Congress of Quantum Chemistry

Menton (France), July 2nd to Sth, 1991

Organized by the International Academy of Quantum Molecular Sciences.

List of Symposia: Dynamics \& Photophysics Molecular Magnets \& Molccular Superconductors; Computers \& New Algorithms; Organometallic \& Bioinorganic Chemistry; Density Oriented Approaches; Organic Reaction Mechanisms; Methods in Theoretical Organic Chemistry; From Clusters to Molecular Solids. Plenary lectures by W. A. Goddard, R. Hoffmann, W. Kohn, W. Kutzelnigg, J. P. Malrieu K. A. Müller, J. A. Pople, S. Shaik, K. Wiberg, and R. A. Wyatt. For further information contact: Prof. L. Salem, Lab. de Chimie Thćorique, Bât. 490, Univ. de Paris Sud, F-91405 Orsay Cedex, France (Tel: (33-1) 69417398. EM: SALEM@FRCTHOII.BITNET Fax: (33-1)69416172)

Chimia 44 (1990) 259-260

(C) Schweiz. Chemiker-Verband; ISSN 00094293

\title{
ALCHEMY II: Molecular Modeling auf dem PC
}

\section{Heinrich G. Bührer*}

\section{ALCHEMY II, Version 2.0I}

TRIPOS Associates, St. Louis, MO 63144 USA; Lieferant: Evans \& Sutherland Computer GmbH, Stahlgruberring 32, D8000 München 82

IBM-PC, XT, AT oder PS/2 oder kompatibler Computer mit mindestens $640 \mathrm{kB}$ RAM, Harddisk, Maus, mathematischem Koprozessor und EGA-Bildschirm Hewlett-Packard ColorPro Graphik-Plotter (sollen auch Kugel-Stäbchen-Modelle geplottet werden, ist eine Graphik-Erweiterung erforderlich)

Preis: US\$ 450.- (für Schulen), sonst US\$ 750.-, Mengenrabatte erhältlich

Zwei 3,5"-Disketten, ein Handbuch. Demodiskette erhältlich.
Apple Macintosh II, IIx oder IIcx mit mindestens 2 MB RAM. Preis: US\$ 950.-, Mengenrabatte erhältlich.

Getestet wurde die IBM-Version auf einem IBM-PS/2 Modell 50

\section{Einleitung}

ALCHEMY II gehört zur Gruppe der Molecular Modeling Software. Vom gleichen Hersteller stammt das modular aufgebaute Programm SYBYL, das auf Grossrechnern läuft. Demgegenüber ist ALCHEMY II eine einfachere, bereits auf einem Personal Computer lauffähige Software. Im Gegensatz zu älteren Program- men wie Molecular Graphics on the IBM PC Microcomputer (Academic Press) erlaubt es auch das direkte Zeichnen von Molekülen ohne Koordinateneingabe.

\section{Programmaufbau}

Die Installation des Programms auf der Harddisk ist problemlos. Das Hauptmenu ist wie folgt aufgebaut:

Im Molekülfenster werden Moleküle gezeichnet, Programmkonfigurationen durchgeführt und der Hilfstext wiedergegeben. Das Menüfenster umfasst u.a. die folgenden Funktionen:

\footnotetext{
* Korrespondenz: Prof. H. G. Bührer Technikum Winterthur Ingenieurschule Abteilung für Chemie Postfach
}

$\mathrm{CH}-8401$ Winterthur 
(112.8 pm for $\mathrm{CO}$ ). This will not be the case in more complicated molecules as you will see below. For $\mathrm{CO}$, it is clear that the energy will become zero at $r_{0}$, i.e. the molecule is free of 'strain'. Again this will be different in more complicated cases.

Now let us see, how we could extend our mechanical model to also predict the structure of $\mathrm{CO}_{2}$, which has longer bond-lengths $(116.0 \mathrm{pm})$. We could assume that there is a repulsive force between atoms which are not directly bound. We could e.g. use the following assumption for this part of the potential:

$\mathrm{E}_{\mathrm{r}}=\mathrm{c}_{2} \mathrm{e}^{-\mathrm{r}^{\prime}}$

$r^{\prime}$ is the distance between nonbonded atoms in our case between the two oxygens. $c_{2}$ is again an empirical parameter, which is stored in the program. Now the total energy for this molecule reads as:

$E=2 E_{s}+E_{r}=2 c_{1}\left(r-r_{0}\right)^{2}+c_{2} e^{-r}$

Again the program can calculate $E$, having $r$ and $r^{\prime}$ from the input data and it will find an energy minimum by systematically changing the distances. This time the final distance will be slightly longer due to the last term and the energy will not be exactly zero, which means that there will be some 'strain' in this molecule. 'Strain in $\mathrm{CO}_{2}$ ' you might ask. Yes, but from the point of view of this force field, not in the usual chemical sense (the above force field is any- way not a very realistic one, but rather a simplified model designed to show the basic ideas). Such a simple example as this could easily be handled with paper and pencil, but medium sized molecules already need the help of a computer.

Now, what do we mean by a force field? The force field is generated from a set of assumptions using a necessary number of parameters. As you can imagine, the force field is the critical part of a molecular mechanics model. It depends on the force field how well experimental structures or enthalpies are modeled, and also how meaningful in the chemical sense, things like strain can be interpreted. Therefore, we will discuss in a later column aspects of force fields in more details.

Perhaps you are now eager to get experience on your personal computer. This would surely be the best way to get acquainted with molecular modeling. There are several good programs available which implement these two concepts, and you will find reviews about such programs in our column. Now, are you on your way?

\section{Announcements}

Workshop on Molecular Mechanics and Molecular Dynamics Simulations

Lausanne, October 2nd and 3rd, 1990

Organized by the Group of Swiss Computationa Chemists (GSCC). Participants limited to 30 . Thi

workshop is intended to have a triple purpose: i) to present the basic features of the physical principles and mathematical formalism of these theoretical models ii) to revicw their most important applications to chemistry and biology; iii) to enable the participants to have a first start in the practice of these techniques by performing some guided calculations. Lectures by $\mathrm{H}$ J.C. Berendsen, Univ of Groningen, M. Dobler. ETH Zürich, and S. Wodak, Free Univ, of Brussels. For further information contact: Prof. J. Weber, Dept. of Physical Chemistry, University of Geneva, 30 , quai Ernest Ansermet, $\mathrm{CH}-1211$ Geneva 4 (Tel. 022/7026530; EM: WEBER@SC2A.UNIGE.CH Fax: 022296102 ).

VIIth International Congress of Quantum Chemistry

Menton (France), July 2nd to Sth, 1991

Organized by the International Academy of Quantum Molecular Sciences.

List of Symposia: Dynamics \& Photophysics Molecular Magnets \& Molccular Superconductors; Computers \& New Algorithms; Organometallic \& Bioinorganic Chemistry; Density Oriented Approaches; Organic Reaction Mechanisms; Methods in Theoretical Organic Chemistry; From Clusters to Molecular Solids. Plenary lectures by W. A. Goddard, R. Hoffmann, W. Kohn, W. Kutzelnigg, J. P. Malrieu K. A. Müller, J. A. Pople, S. Shaik, K. Wiberg, and R. A. Wyatt. For further information contact: Prof. L. Salem, Lab. de Chimie Thćorique, Bât. 490, Univ. de Paris Sud, F-91405 Orsay Cedex, France (Tel: (33-1) 69417398. EM: SALEM@FRCTHOII.BITNET Fax: (33-1)69416172)

Chimia 44 (1990) 259-260

(C) Schweiz. Chemiker-Verband; ISSN 00094293

\section{ALCHEMY II: Molecular Modeling auf dem PC}

\section{Heinrich G. Bührer*}

\section{ALCHEMY II, Version 2.0I}

TRIPOS Associates, St. Louis, MO 63144 USA; Lieferant: Evans \& Sutherland Computer GmbH, Stahlgruberring 32, D8000 München 82

IBM-PC, XT, AT oder PS/2 oder kompatibler Computer mit mindestens $640 \mathrm{kB}$ RAM, Harddisk, Maus, mathematischem Koprozessor und EGA-Bildschirm Hewlett-Packard ColorPro Graphik-Plotter (sollen auch Kugel-Stäbchen-Modelle geplottet werden, ist eine Graphik-Erweiterung erforderlich)

Preis: US\$ 450.- (für Schulen), sonst US\$ 750.-, Mengenrabatte erhältlich

Zwei 3,5"-Disketten, ein Handbuch. Demodiskette erhältlich.
Apple Macintosh II, IIx oder IIcx mit mindestens 2 MB RAM. Preis: US\$ 950.-, Mengenrabatte erhältlich.

Getestet wurde die IBM-Version auf einem IBM-PS/2 Modell 50

\section{Einleitung}

ALCHEMY II gehört zur Gruppe der Molecular Modeling Software. Vom gleichen Hersteller stammt das modular aufgebaute Programm SYBYL, das auf Grossrechnern läuft. Demgegenüber ist ALCHEMY II eine einfachere, bereits auf einem Personal Computer lauffähige Software. Im Gegensatz zu älteren Program- men wie Molecular Graphics on the IBM PC Microcomputer (Academic Press) erlaubt es auch das direkte Zeichnen von Molekülen ohne Koordinateneingabe.

\section{Programmaufbau}

Die Installation des Programms auf der Harddisk ist problemlos. Das Hauptmenu ist wie folgt aufgebaut:

Im Molekülfenster werden Moleküle gezeichnet, Programmkonfigurationen durchgeführt und der Hilfstext wiedergegeben. Das Menüfenster umfasst u.a. die folgenden Funktionen:

\footnotetext{
* Korrespondenz: Prof. H. G. Bührer Technikum Winterthur Ingenieurschule Abteilung für Chemie Postfach
}

$\mathrm{CH}-8401$ Winterthur 


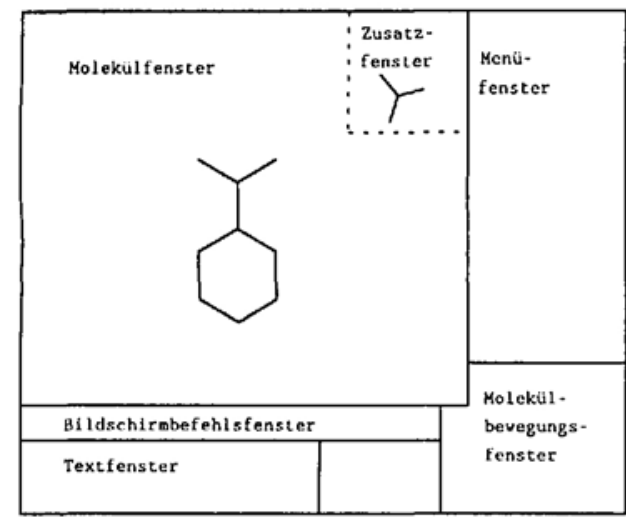

Fig. 1. ALCHEMY-Bildschirm

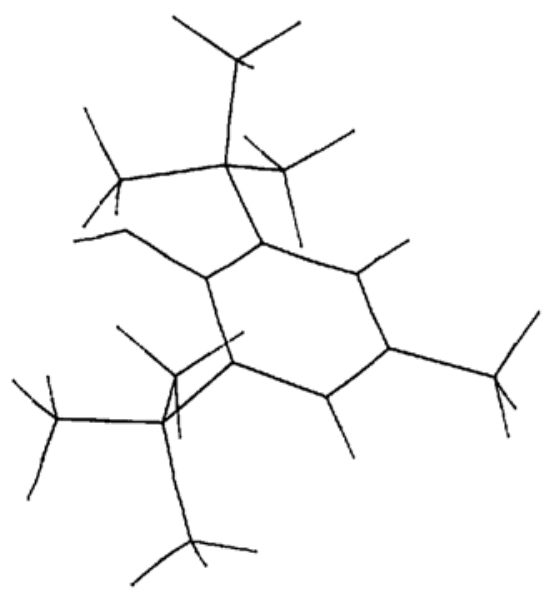

Fig. 2. BHT-Molekül in Dreiding-Darstellung nach 40 Iterationsschritten (leicht gedreht)

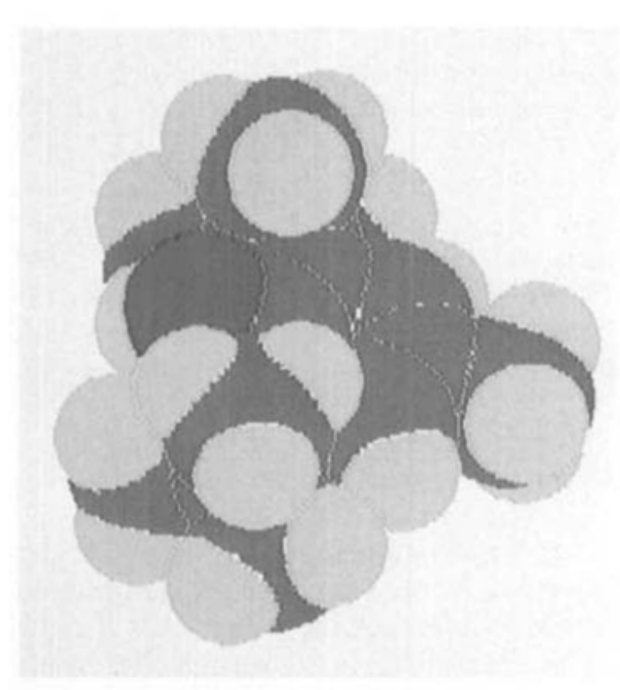

Fig. 3. Kalottenmodell von BHT
- Build and Edit

- Get/Put Molecule

- Delete Molecule

- Center Molecule

- Color

- Measure

- Add/Delete Hydrogens

- Twist/Monitor

- Chiral/Invert

- Minimize

- Fit

- Spacefill

- Ball and Stick

- Plot

- Sybyl Interface

- Crystal Input

Das Textfenster dient beispielsweise zur Eingabe von Dateinamen.

\section{Arbeiten mit dem Programm}

Im Folgenden soll gezeigt werden, wie ein einfaches Molekül, nämlich $B H T$ (Butylhydroxytoluol; 2,6-Di(tert-butyl)-4-methylphenol) aufgebaut wird:

Mit der Funktion Build and Edit wird im Menüfenster auf drei Seiten eine Liste von etwa 50 Fragmenten aufgezeigt. Es sind nebst Atomen auch Ringe, Aminosäuren und Nukleinsäuren vordefiniert. Das Phenylfragment wird angewählt und erscheint als Dreidingmodell mit freien Valenzen im Zusatzfenster (die Grösse dieses Fensters lässt sich im Bildschirmbefehlsfenster anpassen). Durch Anklicken mit der Maus springt der Phenylring ins Molekülfenster. Im Menü wird jetzt das C3-Fragment (C3 bedeutet $\mathrm{sp}^{3}$-hybridisierter Kohlenstoff) ausgewählt und an den betreffenden $\mathrm{Va}$ lenzen am Phenylring mehrfach 'angeklickt'. Um jede neue Bindung kann rotiert werden. Mit $03\left(=\mathrm{sp}^{3}\right.$-hybridisiert) wird Sauerstoff ausgewählt und schliesslich werden im Hauptmenü mit add hydrogens die Valenzen abgesättigt.

Obwohl es möglich ist, bereits während des Aufbaus eines Moleküls z.B. einen Torsionswinkel numerisch einzugeben ( $a l$ ter torsion), bedürfen sterisch gehinderte Moleküle wie BHT oder Ringe der nachträglichen Bearbeitung. Mit der Funktion Minimize kann die potentielle Energie eines Moleküls (berücksichtigt werden Stretch-, Deformations- und van-derWaals-Interaktionen) bestehend aus bis zu 170 Atomen minimiert werden. Das Resultat nach 40 Iterationsschritten zeigt Fig. 2.

Ein Molekül, ein Fragment oder eine Gruppe kann jederzeit mit Put Molecule gesichert oder mit Get Molecule in das Molekülfenster geholt werden.

Mit der Funktion Measure lässt sich ein Molekül 'ausmessen'. So zeigt obiges BHT-Molekül folgende Werte: Abstand zwischen dem Proton der OH-Gruppe und einem Proton der 4-Methylgruppe: 6,6 $\AA$, Bindungswinkel $\mathrm{H}-\mathrm{O}-\mathrm{C}_{\mathrm{ar}}: 116^{\circ}$, Torsionswinkel $\mathrm{H}-\mathrm{O}-\mathrm{C}_{\mathrm{rr}}-\mathrm{C}_{\mathrm{ar}}: 80$ bzw. $-102^{\circ}$. Sehr wertvoll ist die Funktion Twist/Monitor, mit der nachträglich Bindungsabstände und -winkel beliebig numerisch verändert werden können. Mit Chiral/Invert lässt sich die Chiralität von Molekülen oder Atomen bestimmen und umkehren. Mit Hilfe des Molekülbewegungsfensters lassen sich Moleküle - es können auch mehrere Moleküle gleichzeitig dargestellt werden absolut und relativ zueinander um die $x-$, $y$ - und $z$-Achse drehen oder verschieben. Sie können auch beliebig vergrössert oder verkleinert werden. Im Bildschirmbefehlsfenster lässt sich ein dreidimensionales Bild darstellen. Color ordnet Atomen oder Molekülen bestimmte Farben zu. Spacefill ergibt ein Kalotten-, Ball and Stick ein Kugel-Stäbchen-Modell (Fig. 3).

Eine weitere, für das Molecular Modeling zentrale Fähigkeit des Programms ist die Fit-Funktion. Sie gestattet den räumlichen Vergleich zweier Moleküle. Fuse verbindet zwei Ringe, Crystal Input liest Kristallkoordinaten ein.

\section{Import- und Exportmöglichkeiten}

Aus der Datenbank 'Chemical Abstract STN Express' können vier Millionen Moleküle, die dort mit ihren dreidimensionalen Koordinaten enthalten sind, in ALCHEMY eingelesen werden.

Eine Molekülgraphik ist nicht nur mit dem Plotter erhältlich. Ebenso wichtig ist der Export im HPGL-Format und der Import in verschiedene Textverarbeitungsprogramme. So erhält man aus ALCHEMY-Graphiken (Dreiding- wie auch Kalotten- oder Kugel-Stäbchen-Modelle) mit dem Konvertierungsprogramm 'FROMHPGL' ein Metafile, das in ChemText (Molecular Design) mit Text und Graphik aufbereitet und weiterverwendet werden kann. Die Farbe geht allerdings dabei verloren.

\section{Zusammenfassende Wertung}

Die Bedienung des Programms ist einfach und logisch. Help nützt konkret und schnell, so dass man beinahe ohne das in Englisch geschriebene, wohltuend kurzgefasste Handbuch auskommt. Dass Minimize bei grösseren Molekülen Minuten oder auch Stunden in Anspruch nimmt, kann akzeptiert werden. Wünschenswert wären hingegen ein höherer Bildschirmstandard (VGA), eine breitere Druckerunterstützung und eine grössere Atom- und Fragmentbibliothek.

Wenn man bedenkt, welche Möglichkeiten für sterische Untersuchungen an bekannten und neuen Molekülen dieses auch preislich vernünftige Programm auf einem billigen, in praktisch jedem Laboratorium stehenden PC eröffnet, so kann die Anschaffung jedem, der sich mit stereochemischen Fragestellungen befasst, empfohlen werden. 


\section{Schweizerischer Chemiker-Verband Swiss Association of Chemists}

\section{Protokoll der 71. Generalversammlung}

Freitag, den 27. April 1990, bei Lonza AG, Visp.

Vorsitz: Dr. Walter Graf, Präsident des Schweizerischen Chemiker-Verbandes.

Der Vorsitzende begrüsst die versammelten Mitglieder des SChV. Die fristgerecht zugestellte Traktandenliste wird ohne Änderung genehmigt.

1. Das Protokoll der 70. Generalversammlung vom 14. April 1989 (vgl. CHIMIA 1989, 43, 285) wird genehmigt.

2. Der Jahresbericht des Präsidenten (vgl. CHIMIA 1990, 44, 73) wird diskussionslos genehmigt. Der Präsident gibt bekannt, dass Prof. Werner Richarz auf den Zeitpunkt der GV als Vorstandsmitglied des SChV zurückgetreten ist. Herrn Prof. Richarz sei für die viele Arbeit, die er für den SChV erbracht hat, insbesondere auch für die jahrelange erfolgrcichc Organisation der ILMAC, dem Lebensnerv des SChV, bestens gedankt. Im weiteren teilt der Präsident mit, dass auf 1. April 1990 Herr Prof. C. Ganter, Laboratorium für Organische Chemie, ETH-Zürich, die wissenschaftliche Redaktion der CHIMIA übernommen hat. Die technische Redaktion wird seit 1. April 1990 von Herrn Dr. M.V. Kısakürck und Frau $\mathrm{Ch}$. Scheuss betreut.

3. Der von Herrn B. Zigerlig verlesene Revisionsbericht der Revisoren Gut und Kamber wird einstimmig genehmigt.

4. Dem Vorstand sowie Herrn P. Kunz wird einstimmig Décharge erteilt.

5. Die Generalversammlung schliesst sich dem Vorschlag des Vorstandes an und beschliesst einstimmig, die Mitgliederbeiträge für 1990 wie folgt festzusetzen:

Ordentliche Mitglieder

Studentenmitglieder

Firmenmitglieder

Seniorenmitglieder

Seniorenmitglieder (ohne CHIMIA)

Fr. 80.- wie bisher Fr. 25.- wie bisher Fr. 500.- neu

Fr. 35.-- wie bisher

Fr. 20.- wie bisher

Neu: Die CHIMIA wird Firmenmitgliedern gratis für Firmenmitteilungen (nicht Inserate) zur Verfügung stehen. Diese Mitteilungen sind an den Redaktor der CHIMIA, Prof. C. Ganter, Laboratorium für Organische Chemie, ETH-Zentrum, 8092 Zürich, zu richten.

6. Das Budget 1990 wird einstimmig genehmigt.

7. Das wissenschaftliche Programm sieht für die nächsten Jahre wie folgt aus:

1990: 20./21. September

10. Internationales Makromolekulares Symposium in Interlaken. Chairman: Dr. R. Darms, CibaGeigy

1990: 23. 26. Oktober

ILMAC und Basler Treffen für Chemische Technik in Basel

1991: 18./19. April

GV und Frühjahrstagung des Schweiz. ChemikerVerbandes in Agno/Lugano, Besuch der Tessiner Pharmazeutischen Industrie

1991: 3.- 7.Juni

Internationales HPLC-Symposium in Basel

1991: 22.-26. September

11. Internationales Farbensymposium in Montreux. Chairman: Dr. D. Wyrsch, Ciba-Geigy

1992: 21./22. April

Frühjahrstagung und Fusionsgeneralversammlung in Genf anlässlich des Centenary of the Geneva Conference 1892

1992: 23./24. April

6th Modern Synthetic Methods Seminar in Interlaken. Chairman: Prof. R. Scheffold, Universität Bern
1992: 22.-26. Juli

29. International Conference on Coordination Chemistry in Lausanne

1992: 13.-17. September

International Conference on Medicinal Chemistry in Basel

1992: 16. Oktober

Herbstversammlung der vereinigten Gesellschaften in Bern

Technisches Seminar: EG 1992 und die Schweizer Chemie

evtl. 1992, evtl. 1993

Bioorganisches Seminar

Prof. P. Müller berichtet über das 5. Modern Synthetic Methods Seminar (27./28. April 1989), das den Themen 'Biotransformations in Organic Synthesis, Enantioselective Catalysis with Metal Complexes and Aluminosilicates in Organic Synthesis' gewidmet war (Leitung: Prof. R. Scheffold, Universität Bern). Sowohl aus wissenschaftlicher Sicht wic auch im Hinblick auf die Teilnehmerzah (250 Teilnehmer aus Industrie und Hochschule) war dicses Symposium sehr erfolgreich. Die Vorträge wurden alle in 'Modern Synthetic Methods', vol. 5, Editor: R. Scheffold, Springer-Verlag, publiziert. In der CHIMIA 1989, 43, 278, wurde über dieses Symposium berichtet.

Prof. J. Portmann orientiert über das Technische Seminar, das am 29./30. März 1990 unter seiner Leitung in Fribourg stattgefunden hat und dem Thema 'Innovative neue Produkte' gewidmet war. Diesem Seminar haben 120 Chemiker aus Industrie und Hochschule beigewohnt.

8. Die 1989 durchgeführte Abstimmung über die Kooperation crgab folgendes Resultat: Von den 1060 ausgesandten Stimmzetteln sind 640 wieder eingegangen $(60,4 \%)$. Davon stimmten $98 \%$ für eine Kooperation mit der Schweiz. Chemischen Gesellschaft.

Dieselbe Abstimmung wurde auch von der Schweiz. Chemischen Gesellschaft durchgeführt, deren Mitglieder ebenfalls einer Kooperation zustimmten.

Die Kooperationsverhandlungen werden auf Seiten des Schweiz. Chemiker-Verbandes von Dr. Walter Graf (Präsident) und Dr. Georges Haas (Vizepräsident) geführt, von Seiten der Schweiz. Chemischen Gesellschaft von Prof. W. von Philipsborn und Prof. A. Merbach. Diese Kooperationsverhandlungen haben zum Ziel, den Schweizerischen Chemiker-Verband und die Schweizerische Chemische Gesellschaft zu einer Gesellschaft zusammenzuführen. Dic Verhandlungen beinhalten folgende Arbeiten :

1. Organisations-Struktur der neuen Gesellschaft: Organigramm

Wichtigste Zuständigkeiten

2. Statuten:

Vorgehen zur Auflösung der alten Organisationen

Name für neuc Gesellschaft

Neue Statuten

Neugründung (voraussichtlich am 21.4.1992 in Genf)

Modalitäten: Wahl des neuen Vorstandes

Anpassung bestehender Verträge

3. Reglemente:

Ausarbeitung eines Geschäftsführungsreglementes

Preisreglemente

Zeitschriftenstatut

Richtlinien für Fachsektionen

4. Finanzen/Budget:

Aufnahme Ist-Zustand

Modell-Budget für die neue Gesellschaft

Finanzierung

Neue Leistungen der neuen Gesellschaft

5. Personelle Dotierung der neuen Gesellschaft

Namenliste
Suche

Einarbeitung und Verantwortungsübernahme

6. Termin:

18.10.1990: Abschlussbericht durch beide Vorstände genehmigt.

Die Generalversammlung schliesst sich diskussionslos dem Vorgehen des Vorstandes an.

9. Sowohl der Preis des Schweiz. Chemiker-Verbandes wie auch der Max-Lüthi-Preis konnten anlïsslich der diesjährigen Generalversammlung verliehen werden.

Den Preis des Schweiz. Chemiker-Verbandes erhielt Dr. Armin Reller, Universität Zürich, für seine Arbeiten über dic Erzeugung organischer Verbindungen aus anorganischen Carbonaten und Wasserstoff.

Der Max-Lüthi-Preis konnte Herrn Marcel Sonderegger, Technikum Winterthur, für seine Arbeit 'Kunststoff-Werkstoffe: Datenbanken, Verarbeitung, Charakterisierung' verliehen werden.

10. Der Präsident macht darauf aufmerksam, dass 1991 dic letzte Generalversammlung des Schweiz Chemiker-Verbandes stattfinden wird. Es ist deshalb vorgesehen, eine zweitägige Frühjahrstagung (18./19. April 1991) mit Besuch der Tessiner Pharmazeutischen Industrie durchzuführen. Vorläufiges Programm: Donnerstag, 18.4., Nachmittag: Treffen in Agno und Vorträge von vier Tessiner Pharmazie-Firmen. Gemütlicher Abend. Freitag, 19.4 Morgen: Besuch von 3 Firmen; Nachmittag: ausgedehnte GV und Orientierung über die 1992 zu gründende Nachfolge-Gesellschaft.

Die Generalversammlung schliesst um 13 Uhr.

Für das Protokoll: Beatrice Köchli

\section{Arbeitsgemeinschaft der Schweizerischen Kunststoffindustrie (aski)}

Die aski lädt am 14. September 1990 zur zweiten öffentlichen Tagung, 'Kunststoffe ... cine ökologische Herausforderung, ins Casino Zürich Horn ein. Zur Teilnahme sind neben Umweltbeauftragten aus Bund Kantonen und Industrie auch Konsumentenschutzorganisationen, Umweltorganisationen, Studenten, Medien und weitere Interessenten eingeladen.

Auf der einen Seite sind Kunststoffe zur Lösun vieler Probleme unverzichtbare Werkstoffe geworden. Auf der anderen Seite wird den Kunststoffen vorgeworfen, ihre Verwendung erfordere im Verhältnis zu alternativen Werkstoffen zuviel Energie und die Umwelt werde stärker belastet. Ausserdem verschärft die Menge, die Leichtigkeit sowie die Witterungs- und Altersbeständigkeit der Kunststoffe das ohnehin schon bestehende Entsorgungsproblem.

Die Tagung hat zum Ziel, ökologisch und ökonomisch schlüssige Wege und Konzepte aus diesem Spannungsfeld zu erarbeiten und zu diskutieren. Kompetente Fachleute äussern sich am Vormittag in drei Grundsatzreferaten dazu:

Professor Kollreuter (Basler Arbeitsgruppe für Konjunkturforschung) beleuchtet die zukünftige ökonomische Entwicklung der Kunststoffe; Professor Menges (Technische Hochschule Aachen) den Stand und die Möglichkeiten von Kunststoffrecycling; Professor Imboden (ETH Zürich) spricht zum Thema: Wissenschaft, Technik und Gesellschaft. Aufgelockert werden die Referate durch Einlagen des Schriftstellers und Katbarettisten Martin Hamburger. Eine Diskussion zu of fenen Fragen wird das Morgenprogramm abschliessen.

Das Round Table Gespräch am Nachmittag stcht unter dem Motto: 'Kunststoffe und Ökologie im Kreuzfeuer'. Vertreter der Abfallwirtschaft, Konsumenten, Industrie, Politik und Chemie diskutieren die ökologischen und ökonomischen Anforderungen an die Kunststoffe.

Beim Erfrischungsbuffet am Schluss der Tagung besteht die Möglichkeit zu persönlichen Gesprächen und Begegnungen.

Interessenten, die an der Tagung 'Kunststoffe -- eine ökologische Herausforderung' teilnehmen möchten, wenden sich an das aski-Sekretariat, Nordstrasse 15, 8006 Zürich, Tel. 013633610 


\section{Schweizerischer Chemiker-Verband Swiss Association of Chemists}

\section{Protokoll der 71. Generalversammlung}

Freitag, den 27. April 1990, bei Lonza AG, Visp.

Vorsitz: Dr. Walter Graf, Präsident des Schweizerischen Chemiker-Verbandes.

Der Vorsitzende begrüsst die versammelten Mitglieder des SChV. Die fristgerecht zugestellte Traktandenliste wird ohne Änderung genehmigt.

1. Das Protokoll der 70. Generalversammlung vom 14. April 1989 (vgl. CHIMIA 1989, 43, 285) wird genehmigt.

2. Der Jahresbericht des Präsidenten (vgl. CHIMIA 1990, 44, 73) wird diskussionslos genehmigt. Der Präsident gibt bekannt, dass Prof. Werner Richarz auf den Zeitpunkt der GV als Vorstandsmitglied des SChV zurückgetreten ist. Herrn Prof. Richarz sei für die viele Arbeit, die er für den SChV erbracht hat, insbesondere auch für die jahrelange erfolgrcichc Organisation der ILMAC, dem Lebensnerv des SChV, bestens gedankt. Im weiteren teilt der Präsident mit, dass auf 1. April 1990 Herr Prof. C. Ganter, Laboratorium für Organische Chemie, ETH-Zürich, die wissenschaftliche Redaktion der CHIMIA übernommen hat. Die technische Redaktion wird seit 1. April 1990 von Herrn Dr. M.V. Kısakürck und Frau $\mathrm{Ch}$. Scheuss betreut.

3. Der von Herrn B. Zigerlig verlesene Revisionsbericht der Revisoren Gut und Kamber wird einstimmig genehmigt.

4. Dem Vorstand sowie Herrn P. Kunz wird einstimmig Décharge erteilt.

5. Die Generalversammlung schliesst sich dem Vorschlag des Vorstandes an und beschliesst einstimmig, die Mitgliederbeiträge für 1990 wie folgt festzusetzen:

Ordentliche Mitglieder

Studentenmitglieder

Firmenmitglieder

Seniorenmitglieder

Seniorenmitglieder (ohne CHIMIA)

Fr. 80.- wie bisher Fr. 25.- wie bisher Fr. 500.- neu

Fr. 35.-- wie bisher

Fr. 20.- wie bisher

Neu: Die CHIMIA wird Firmenmitgliedern gratis für Firmenmitteilungen (nicht Inserate) zur Verfügung stehen. Diese Mitteilungen sind an den Redaktor der CHIMIA, Prof. C. Ganter, Laboratorium für Organische Chemie, ETH-Zentrum, 8092 Zürich, zu richten.

6. Das Budget 1990 wird einstimmig genehmigt.

7. Das wissenschaftliche Programm sieht für die nächsten Jahre wie folgt aus:

1990: 20./21. September

10. Internationales Makromolekulares Symposium in Interlaken. Chairman: Dr. R. Darms, CibaGeigy

1990: 23. 26. Oktober

ILMAC und Basler Treffen für Chemische Technik in Basel

1991: 18./19. April

GV und Frühjahrstagung des Schweiz. ChemikerVerbandes in Agno/Lugano, Besuch der Tessiner Pharmazeutischen Industrie

1991: 3.- 7.Juni

Internationales HPLC-Symposium in Basel

1991: 22.-26. September

11. Internationales Farbensymposium in Montreux. Chairman: Dr. D. Wyrsch, Ciba-Geigy

1992: 21./22. April

Frühjahrstagung und Fusionsgeneralversammlung in Genf anlässlich des Centenary of the Geneva Conference 1892

1992: 23./24. April

6th Modern Synthetic Methods Seminar in Interlaken. Chairman: Prof. R. Scheffold, Universität Bern
1992: 22.-26. Juli

29. International Conference on Coordination Chemistry in Lausanne

1992: 13.-17. September

International Conference on Medicinal Chemistry in Basel

1992: 16. Oktober

Herbstversammlung der vereinigten Gesellschaften in Bern

Technisches Seminar: EG 1992 und die Schweizer Chemie

evtl. 1992, evtl. 1993

Bioorganisches Seminar

Prof. P. Müller berichtet über das 5. Modern Synthetic Methods Seminar (27./28. April 1989), das den Themen 'Biotransformations in Organic Synthesis, Enantioselective Catalysis with Metal Complexes and Aluminosilicates in Organic Synthesis' gewidmet war (Leitung: Prof. R. Scheffold, Universität Bern). Sowohl aus wissenschaftlicher Sicht wic auch im Hinblick auf die Teilnehmerzah (250 Teilnehmer aus Industrie und Hochschule) war dicses Symposium sehr erfolgreich. Die Vorträge wurden alle in 'Modern Synthetic Methods', vol. 5, Editor: R. Scheffold, Springer-Verlag, publiziert. In der CHIMIA 1989, 43, 278, wurde über dieses Symposium berichtet.

Prof. J. Portmann orientiert über das Technische Seminar, das am 29./30. März 1990 unter seiner Leitung in Fribourg stattgefunden hat und dem Thema 'Innovative neue Produkte' gewidmet war. Diesem Seminar haben 120 Chemiker aus Industrie und Hochschule beigewohnt.

8. Die 1989 durchgeführte Abstimmung über die Kooperation crgab folgendes Resultat: Von den 1060 ausgesandten Stimmzetteln sind 640 wieder eingegangen $(60,4 \%)$. Davon stimmten $98 \%$ für eine Kooperation mit der Schweiz. Chemischen Gesellschaft.

Dieselbe Abstimmung wurde auch von der Schweiz. Chemischen Gesellschaft durchgeführt, deren Mitglieder ebenfalls einer Kooperation zustimmten.

Die Kooperationsverhandlungen werden auf Seiten des Schweiz. Chemiker-Verbandes von Dr. Walter Graf (Präsident) und Dr. Georges Haas (Vizepräsident) geführt, von Seiten der Schweiz. Chemischen Gesellschaft von Prof. W. von Philipsborn und Prof. A. Merbach. Diese Kooperationsverhandlungen haben zum Ziel, den Schweizerischen Chemiker-Verband und die Schweizerische Chemische Gesellschaft zu einer Gesellschaft zusammenzuführen. Dic Verhandlungen beinhalten folgende Arbeiten :

1. Organisations-Struktur der neuen Gesellschaft: Organigramm

Wichtigste Zuständigkeiten

2. Statuten:

Vorgehen zur Auflösung der alten Organisationen

Name für neuc Gesellschaft

Neue Statuten

Neugründung (voraussichtlich am 21.4.1992 in Genf)

Modalitäten: Wahl des neuen Vorstandes

Anpassung bestehender Verträge

3. Reglemente:

Ausarbeitung eines Geschäftsführungsreglementes

Preisreglemente

Zeitschriftenstatut

Richtlinien für Fachsektionen

4. Finanzen/Budget:

Aufnahme Ist-Zustand

Modell-Budget für die neue Gesellschaft

Finanzierung

Neue Leistungen der neuen Gesellschaft

5. Personelle Dotierung der neuen Gesellschaft

Namenliste
Suche

Einarbeitung und Verantwortungsübernahme

6. Termin:

18.10.1990: Abschlussbericht durch beide Vorstände genehmigt.

Die Generalversammlung schliesst sich diskussionslos dem Vorgehen des Vorstandes an.

9. Sowohl der Preis des Schweiz. Chemiker-Verbandes wie auch der Max-Lüthi-Preis konnten anlïsslich der diesjährigen Generalversammlung verliehen werden.

Den Preis des Schweiz. Chemiker-Verbandes erhielt Dr. Armin Reller, Universität Zürich, für seine Arbeiten über dic Erzeugung organischer Verbindungen aus anorganischen Carbonaten und Wasserstoff.

Der Max-Lüthi-Preis konnte Herrn Marcel Sonderegger, Technikum Winterthur, für seine Arbeit 'Kunststoff-Werkstoffe: Datenbanken, Verarbeitung, Charakterisierung' verliehen werden.

10. Der Präsident macht darauf aufmerksam, dass 1991 dic letzte Generalversammlung des Schweiz Chemiker-Verbandes stattfinden wird. Es ist deshalb vorgesehen, eine zweitägige Frühjahrstagung (18./19. April 1991) mit Besuch der Tessiner Pharmazeutischen Industrie durchzuführen. Vorläufiges Programm: Donnerstag, 18.4., Nachmittag: Treffen in Agno und Vorträge von vier Tessiner Pharmazie-Firmen. Gemütlicher Abend. Freitag, 19.4 Morgen: Besuch von 3 Firmen; Nachmittag: ausgedehnte GV und Orientierung über die 1992 zu gründende Nachfolge-Gesellschaft.

Die Generalversammlung schliesst um 13 Uhr.

Für das Protokoll: Beatrice Köchli

\section{Arbeitsgemeinschaft der Schweizerischen Kunststoffindustrie (aski)}

Die aski lädt am 14. September 1990 zur zweiten öffentlichen Tagung, 'Kunststoffe ... cine ökologische Herausforderung, ins Casino Zürich Horn ein. Zur Teilnahme sind neben Umweltbeauftragten aus Bund Kantonen und Industrie auch Konsumentenschutzorganisationen, Umweltorganisationen, Studenten, Medien und weitere Interessenten eingeladen.

Auf der einen Seite sind Kunststoffe zur Lösun vieler Probleme unverzichtbare Werkstoffe geworden. Auf der anderen Seite wird den Kunststoffen vorgeworfen, ihre Verwendung erfordere im Verhältnis zu alternativen Werkstoffen zuviel Energie und die Umwelt werde stärker belastet. Ausserdem verschärft die Menge, die Leichtigkeit sowie die Witterungs- und Altersbeständigkeit der Kunststoffe das ohnehin schon bestehende Entsorgungsproblem.

Die Tagung hat zum Ziel, ökologisch und ökonomisch schlüssige Wege und Konzepte aus diesem Spannungsfeld zu erarbeiten und zu diskutieren. Kompetente Fachleute äussern sich am Vormittag in drei Grundsatzreferaten dazu:

Professor Kollreuter (Basler Arbeitsgruppe für Konjunkturforschung) beleuchtet die zukünftige ökonomische Entwicklung der Kunststoffe; Professor Menges (Technische Hochschule Aachen) den Stand und die Möglichkeiten von Kunststoffrecycling; Professor Imboden (ETH Zürich) spricht zum Thema: Wissenschaft, Technik und Gesellschaft. Aufgelockert werden die Referate durch Einlagen des Schriftstellers und Katbarettisten Martin Hamburger. Eine Diskussion zu of fenen Fragen wird das Morgenprogramm abschliessen.

Das Round Table Gespräch am Nachmittag stcht unter dem Motto: 'Kunststoffe und Ökologie im Kreuzfeuer'. Vertreter der Abfallwirtschaft, Konsumenten, Industrie, Politik und Chemie diskutieren die ökologischen und ökonomischen Anforderungen an die Kunststoffe.

Beim Erfrischungsbuffet am Schluss der Tagung besteht die Möglichkeit zu persönlichen Gesprächen und Begegnungen.

Interessenten, die an der Tagung 'Kunststoffe -- eine ökologische Herausforderung' teilnehmen möchten, wenden sich an das aski-Sekretariat, Nordstrasse 15, 8006 Zürich, Tel. 013633610 
Schweizerischer Chemiker-Verband Swiss Association of Chemists

Polymer-Gruppe der Schweiz

Polymer Group of Switzerland

Schweizerische Chemische Gesellschaft

Swiss Chemical Society

\section{0th International Macromolecular Symposium}

September 20/21, 1990, Interlaken - Switzerland

\section{Polymer Surfaces and Interfaces - Key to} High Performance Materials

\section{Chairman: Dr. Roland Darms} Geigy AG, Marly/Fribourg; Prof. H. J. Kausch, Ecole Polytechnique Fédérale Lausanne; Prof. J. Meissner, Eidg. Technische Hochschule Zürich; Prof. P. Müller, Schweizerischer Chemiker-Verband; Prof. M. Neuenschwander, Universität Bern; Prof. P. Pino †, Eidg. Technische Hochschule Zürich; Prof. U. Suter, Eidg Technische Hochschule Zürich; Dr. H.J. Schultze,

Programme

9.00

Opening of the Symposium

General

9.15-10.15 Prof. G. M. Whitesides, Harvard University, Department of Chemistry, Cambridge, USA

'The Physical-Organic Chemistry of Interfaces'

$10.15 \quad 10.45 \quad$ Coffee break
Scientific Committee: Dr. Roland Darms, CibaEMS-Chemie, Domat/Ems.

September 20, 1990
$10.45-11.45$

Dr. Th. Russell, IBM Almaden Research Center, San Jose, USA

'Methods for the Characterization of Polymer Surfaces and Interfaces'

11.45-12.45 Prof. G. Wegner, Max Planck-Institut für Polymerforschung, Mainz, FRG 'Molecular Architecture of Well Defined Surfaces and Interphases of Organic and Polymer Materials'

Lunch

15.00-16.00 Prof. T.J. McCarthy, University of Massachusetts at Amherst, Polyme Science and Engineering, Amherst, USA

'Organic Chemistry at Chemically Resistant Polymer Surfaces. Modification of Surface Reactivity and Surface Properties'

$16.00-16.30$

Coffee break

Adhesion/Adhesives

16.30-17.30 Dr. A.J. Kinloch, Department of Mechanical Engineering, Imperial College, London, GB

'Adhesive Bonding: The Importance of Polymeric Interfaces'

17.30-18.10 Dr. K. Jud, EMS TOGO AG, Romanshorn, Switzerland

'Improvement of Adhesion on Automotive Top Coats by Corona Discharge'
September 21, 1990

\section{Coatings}

08.30-09.30 Dr J.M. Loutz, UCB, Specialty Chemicals Division, Drogenbos, Belgium 'Recent Developments in the Field of Powder Coatings'

09.30-10.10 Dr. H. Liertz, Siemens AG, München, FRG

'The Influence of Coatings on the Fracture Behaviour of Light WaveGuides'

\subsection{0-10.45 Coffee break}

Filled Polymer-Systems

10.45-11.45 Dr. T. Kaiser, Asea Brown Boveri, Research Center, Baden, Switzerland 'Role of Particle-Matrix Interface in the Deformation and Fracture Behaviour of Filled Epoxy Resins

11.45-12.25 Dr. H.P. Schlumpf, Plüss-Staufer AG, Oftringen, Switzerland

'Physico-Chemical Aspects of Filers in Polypropylene'

12.25-13.30 Business Lunch

Composites

13.30-14.30 Dr. R.F. Siegmund, Ciba-Geigy Corporation, Composite Materials Department, Anaheim, USA

'Some Newer Applications of Advanced Polymer Composites'

14.30-15.10 Prof. A. Pavan, Dipartimento di Chimica Industriale e Ingegneria Chimica, Politecnico di Milano, Milano, Italy "A Study on the Toughening of Polymer Matrix Composites by Interphase Modification'

\section{Registration}

The registration card should be sent to: Secretary's Office for SAC-Symposia, Institute of Organic Chemistry, University of Berne, Freiestrasse 3, CH-3012 Berne, Switzerland, Tel. 031654311 , Telex 912406 uni Mixer at the Hotel Victoria Jungfrau ch, Fax 031 654499.

\section{Schweizerischer Wissenschaftsrat, Sekretariat Conseil suisse de la science, Secrétariat Consiglio svizzero della scienza, Segreteria}

\section{Forschung und Zukunft}

Empfehlungen des Schweizerischen Wissenschaftsrates für die Periode 19921995

Schwerpunkte auf den Forschungsgebieten Umwelt, soziopolitische Probleme, Technologie, eine konkurrenzfähigere Forschung auf internationaler Ebene; quantifizierte Vorschläge; Optionen im Bereich Forschungsmanagement: so lassen sich die Empfehlungen des Schweizerischen Wissenschaftsrates (SWR) für di Zukunft kurz umreissen.

Der Schweizerische Wissenschaftsrat hat soeben seine Empfehlungen für die schweizerische Forschungspolitik veröffentlicht. Diese dienten als Grundlage für die 'Ziele', die der Bundesrat vor kurzem angenommen hat. Fundierte Analysen und ein reichhaltiges Material sind Teil der Empfehlungen (Forschungsplatz Schweiz. Horizont 1995, SWR 1989, Bern 1990).

-- Umwelt/soziopolitische Probleme/Technologie: dre prioritäre Forschungsbereiche, die aber nicht auf Kosten der Grundlagenforschung bearbeitet werden sollen.

Der SWR unterstreicht vor allem folgende Forschungsbereiche, die in unserem Land noch ungenügend entwickelt sind: 1 . Schutz der Umwelt. 2. Bewältigung soziopolitischer Probleme wie Überalterung, Bitdung, Gesundheit (insbesondere in Bezug auf AIDS) 3. Neue Technologien und ihre Auswirkungen. Gleichzeitig hebt er hervor, dass die Grundlagenforschung der Grundstein jeglicher Aktivität im Bereich Forschung und Entwicklung $(\mathrm{F}+\mathrm{E})$ bleibt.

- Konkurrenzfähige Forschung auf internationaler Ebene

Der Bund muss die Bemühungen auf dem Gebiete von $F+E$ unterstützen. Im Hinblick auf das zukünftige Europa ist es sogar eine seiner Hauptaufgaben, die Forschung nicht nur für die eigenen Bedürfnisse zu fördern, sondern auch, um auf internationaler Ebene eine starke Position zu behalten.

Zahlen und konkrete Vorschläge der SWR

Der SWR hatte den Mut, die zusätzlichen Forschungsbedürfnisse für die Periode 1992-1995 in Zahlen zu nennen. So muss der Bund gewillt sein, seine Ausgaben für die Forschung, die sich bis anhin auf 1,5 Mia. Fr. pro Jahr beliefen (Nationalfonds, Hochschulen, Forschung im Rahmen der Bundesämter, internationale Engagements etc.) um 8 bis $10 \%$ pro Jah zu erhöhen. Momentan wird weit weniger als ein Viertel der Forschung von der öffentlichen Hand finanziert, der Rest wird von der Privatwirtschaft übernommen. Dieser Anteil ist einer der niedrigsten im internationalen Vergleich.

- Wie soll die Forschung verwaltet werden: freie Forschung vs. orientierte Forschung

Der SWR hatte ebenfalls den Mut, eine klare Grenzlinie zu ziehen zwischen der vom Staat orientierten Forschung, die auf die Bedürfnisse von Wirtschaft und Gesellschaft eingehen muss, und der Forschung, die frei bleiben muss, um ihrer Hauptaufgabe, neue Er- kenntnisse zu erbringen, gerecht zu werden. Mit einem besseren Forschungsmanagement sollen beide Forschungstypen verbessert werden: Planung, Koordination, Internationale Zusammenarbeit, Zusammenarbeit zwischen öffentlicher und privater Forschung, frühzeitiges Erkennen möglicher Forschungsbereiche, Evaluation der Resultate und Konsequenzen der Forschung und deren technischen Anwendungen.

Quelle: Forschungsplatz Schweiz. Horizont 1995, SWR 1989, Bern 1990.

Für weitere Auskünfte: Dr. François Da Pozzo Dr. Edo Poglia

Sekretariat des Schweizerischen Wissenschaftsrates Wildhainweg 9, 3001 Bern Telephon: 031619666

\section{IUPAC-Empfehlungen}

Nomenclature, symbols, units and their usage in spectrochemical analysis - XII. Terms related to electrothermal atomization

Terminology for electrothermal atomization is recommended and its use illustrated mainly in the application of electrothermal atomization to atomic absorption spectrometry. The terms, symbols and abbreviations required for the description of atomizers, for the processes involved in electrothermal atomization and in their analytical use are prescribed. An index of terms is included.

Comments on the document should be sent by Junc 30, 1991 to: Dr. T. A. M. Ure, Department of Pure and Applied Chemistry, Thomas Graham Building, University of Strathclyde, 295 Cathedral Street, Glasgow GI 1XL, UK. 
Schweizerischer Chemiker-Verband Swiss Association of Chemists

Polymer-Gruppe der Schweiz

Polymer Group of Switzerland

Schweizerische Chemische Gesellschaft

Swiss Chemical Society

\section{0th International Macromolecular Symposium}

September 20/21, 1990, Interlaken - Switzerland

\section{Polymer Surfaces and Interfaces - Key to} High Performance Materials

\section{Chairman: Dr. Roland Darms} Geigy AG, Marly/Fribourg; Prof. H. J. Kausch, Ecole Polytechnique Fédérale Lausanne; Prof. J. Meissner, Eidg. Technische Hochschule Zürich; Prof. P. Müller, Schweizerischer Chemiker-Verband; Prof. M. Neuenschwander, Universität Bern; Prof. P. Pino †, Eidg. Technische Hochschule Zürich; Prof. U. Suter, Eidg Technische Hochschule Zürich; Dr. H.J. Schultze,

Programme

9.00

Opening of the Symposium

General

9.15-10.15 Prof. G. M. Whitesides, Harvard University, Department of Chemistry, Cambridge, USA

'The Physical-Organic Chemistry of Interfaces'

$10.15 \quad 10.45 \quad$ Coffee break
Scientific Committee: Dr. Roland Darms, CibaEMS-Chemie, Domat/Ems.

September 20, 1990
$10.45-11.45$

Dr. Th. Russell, IBM Almaden Research Center, San Jose, USA

'Methods for the Characterization of Polymer Surfaces and Interfaces'

11.45-12.45 Prof. G. Wegner, Max Planck-Institut für Polymerforschung, Mainz, FRG 'Molecular Architecture of Well Defined Surfaces and Interphases of Organic and Polymer Materials'

Lunch

15.00-16.00 Prof. T.J. McCarthy, University of Massachusetts at Amherst, Polyme Science and Engineering, Amherst, USA

'Organic Chemistry at Chemically Resistant Polymer Surfaces. Modification of Surface Reactivity and Surface Properties'

$16.00-16.30$

Coffee break

Adhesion/Adhesives

16.30-17.30 Dr. A.J. Kinloch, Department of Mechanical Engineering, Imperial College, London, GB

'Adhesive Bonding: The Importance of Polymeric Interfaces'

17.30-18.10 Dr. K. Jud, EMS TOGO AG, Romanshorn, Switzerland

'Improvement of Adhesion on Automotive Top Coats by Corona Discharge'
September 21, 1990

\section{Coatings}

08.30-09.30 Dr J.M. Loutz, UCB, Specialty Chemicals Division, Drogenbos, Belgium 'Recent Developments in the Field of Powder Coatings'

09.30-10.10 Dr. H. Liertz, Siemens AG, München, FRG

'The Influence of Coatings on the Fracture Behaviour of Light WaveGuides'

\subsection{0-10.45 Coffee break}

Filled Polymer-Systems

10.45-11.45 Dr. T. Kaiser, Asea Brown Boveri, Research Center, Baden, Switzerland 'Role of Particle-Matrix Interface in the Deformation and Fracture Behaviour of Filled Epoxy Resins

11.45-12.25 Dr. H.P. Schlumpf, Plüss-Staufer AG, Oftringen, Switzerland

'Physico-Chemical Aspects of Filers in Polypropylene'

12.25-13.30 Business Lunch

Composites

13.30-14.30 Dr. R.F. Siegmund, Ciba-Geigy Corporation, Composite Materials Department, Anaheim, USA

'Some Newer Applications of Advanced Polymer Composites'

14.30-15.10 Prof. A. Pavan, Dipartimento di Chimica Industriale e Ingegneria Chimica, Politecnico di Milano, Milano, Italy "A Study on the Toughening of Polymer Matrix Composites by Interphase Modification'

\section{Registration}

The registration card should be sent to: Secretary's Office for SAC-Symposia, Institute of Organic Chemistry, University of Berne, Freiestrasse 3, CH-3012 Berne, Switzerland, Tel. 031654311 , Telex 912406 uni Mixer at the Hotel Victoria Jungfrau ch, Fax 031 654499.

\section{Schweizerischer Wissenschaftsrat, Sekretariat Conseil suisse de la science, Secrétariat Consiglio svizzero della scienza, Segreteria}

\section{Forschung und Zukunft}

Empfehlungen des Schweizerischen Wissenschaftsrates für die Periode 19921995

Schwerpunkte auf den Forschungsgebieten Umwelt, soziopolitische Probleme, Technologie, eine konkurrenzfähigere Forschung auf internationaler Ebene; quantifizierte Vorschläge; Optionen im Bereich Forschungsmanagement: so lassen sich die Empfehlungen des Schweizerischen Wissenschaftsrates (SWR) für di Zukunft kurz umreissen.

Der Schweizerische Wissenschaftsrat hat soeben seine Empfehlungen für die schweizerische Forschungspolitik veröffentlicht. Diese dienten als Grundlage für die 'Ziele', die der Bundesrat vor kurzem angenommen hat. Fundierte Analysen und ein reichhaltiges Material sind Teil der Empfehlungen (Forschungsplatz Schweiz. Horizont 1995, SWR 1989, Bern 1990).

-- Umwelt/soziopolitische Probleme/Technologie: dre prioritäre Forschungsbereiche, die aber nicht auf Kosten der Grundlagenforschung bearbeitet werden sollen.

Der SWR unterstreicht vor allem folgende Forschungsbereiche, die in unserem Land noch ungenügend entwickelt sind: 1 . Schutz der Umwelt. 2. Bewältigung soziopolitischer Probleme wie Überalterung, Bitdung, Gesundheit (insbesondere in Bezug auf AIDS) 3. Neue Technologien und ihre Auswirkungen. Gleichzeitig hebt er hervor, dass die Grundlagenforschung der Grundstein jeglicher Aktivität im Bereich Forschung und Entwicklung $(\mathrm{F}+\mathrm{E})$ bleibt.

- Konkurrenzfähige Forschung auf internationaler Ebene

Der Bund muss die Bemühungen auf dem Gebiete von $F+E$ unterstützen. Im Hinblick auf das zukünftige Europa ist es sogar eine seiner Hauptaufgaben, die Forschung nicht nur für die eigenen Bedürfnisse zu fördern, sondern auch, um auf internationaler Ebene eine starke Position zu behalten.

Zahlen und konkrete Vorschläge der SWR

Der SWR hatte den Mut, die zusätzlichen Forschungsbedürfnisse für die Periode 1992-1995 in Zahlen zu nennen. So muss der Bund gewillt sein, seine Ausgaben für die Forschung, die sich bis anhin auf 1,5 Mia. Fr. pro Jahr beliefen (Nationalfonds, Hochschulen, Forschung im Rahmen der Bundesämter, internationale Engagements etc.) um 8 bis $10 \%$ pro Jah zu erhöhen. Momentan wird weit weniger als ein Viertel der Forschung von der öffentlichen Hand finanziert, der Rest wird von der Privatwirtschaft übernommen. Dieser Anteil ist einer der niedrigsten im internationalen Vergleich.

- Wie soll die Forschung verwaltet werden: freie Forschung vs. orientierte Forschung

Der SWR hatte ebenfalls den Mut, eine klare Grenzlinie zu ziehen zwischen der vom Staat orientierten Forschung, die auf die Bedürfnisse von Wirtschaft und Gesellschaft eingehen muss, und der Forschung, die frei bleiben muss, um ihrer Hauptaufgabe, neue Er- kenntnisse zu erbringen, gerecht zu werden. Mit einem besseren Forschungsmanagement sollen beide Forschungstypen verbessert werden: Planung, Koordination, Internationale Zusammenarbeit, Zusammenarbeit zwischen öffentlicher und privater Forschung, frühzeitiges Erkennen möglicher Forschungsbereiche, Evaluation der Resultate und Konsequenzen der Forschung und deren technischen Anwendungen.

Quelle: Forschungsplatz Schweiz. Horizont 1995, SWR 1989, Bern 1990.

Für weitere Auskünfte: Dr. François Da Pozzo Dr. Edo Poglia

Sekretariat des Schweizerischen Wissenschaftsrates Wildhainweg 9, 3001 Bern Telephon: 031619666

\section{IUPAC-Empfehlungen}

Nomenclature, symbols, units and their usage in spectrochemical analysis - XII. Terms related to electrothermal atomization

Terminology for electrothermal atomization is recommended and its use illustrated mainly in the application of electrothermal atomization to atomic absorption spectrometry. The terms, symbols and abbreviations required for the description of atomizers, for the processes involved in electrothermal atomization and in their analytical use are prescribed. An index of terms is included.

Comments on the document should be sent by Junc 30, 1991 to: Dr. T. A. M. Ure, Department of Pure and Applied Chemistry, Thomas Graham Building, University of Strathclyde, 295 Cathedral Street, Glasgow GI 1XL, UK. 
Schweizerischer Chemiker-Verband Swiss Association of Chemists

Polymer-Gruppe der Schweiz

Polymer Group of Switzerland

Schweizerische Chemische Gesellschaft

Swiss Chemical Society

\section{0th International Macromolecular Symposium}

September 20/21, 1990, Interlaken - Switzerland

\section{Polymer Surfaces and Interfaces - Key to} High Performance Materials

\section{Chairman: Dr. Roland Darms} Geigy AG, Marly/Fribourg; Prof. H. J. Kausch, Ecole Polytechnique Fédérale Lausanne; Prof. J. Meissner, Eidg. Technische Hochschule Zürich; Prof. P. Müller, Schweizerischer Chemiker-Verband; Prof. M. Neuenschwander, Universität Bern; Prof. P. Pino †, Eidg. Technische Hochschule Zürich; Prof. U. Suter, Eidg Technische Hochschule Zürich; Dr. H.J. Schultze,

Programme

9.00

Opening of the Symposium

General

9.15-10.15 Prof. G. M. Whitesides, Harvard University, Department of Chemistry, Cambridge, USA

'The Physical-Organic Chemistry of Interfaces'

$10.15 \quad 10.45 \quad$ Coffee break
Scientific Committee: Dr. Roland Darms, CibaEMS-Chemie, Domat/Ems.

September 20, 1990
$10.45-11.45$

Dr. Th. Russell, IBM Almaden Research Center, San Jose, USA

'Methods for the Characterization of Polymer Surfaces and Interfaces'

11.45-12.45 Prof. G. Wegner, Max Planck-Institut für Polymerforschung, Mainz, FRG 'Molecular Architecture of Well Defined Surfaces and Interphases of Organic and Polymer Materials'

Lunch

15.00-16.00 Prof. T.J. McCarthy, University of Massachusetts at Amherst, Polyme Science and Engineering, Amherst, USA

'Organic Chemistry at Chemically Resistant Polymer Surfaces. Modification of Surface Reactivity and Surface Properties'

$16.00-16.30$

Coffee break

Adhesion/Adhesives

16.30-17.30 Dr. A.J. Kinloch, Department of Mechanical Engineering, Imperial College, London, GB

'Adhesive Bonding: The Importance of Polymeric Interfaces'

17.30-18.10 Dr. K. Jud, EMS TOGO AG, Romanshorn, Switzerland

'Improvement of Adhesion on Automotive Top Coats by Corona Discharge'
September 21, 1990

\section{Coatings}

08.30-09.30 Dr J.M. Loutz, UCB, Specialty Chemicals Division, Drogenbos, Belgium 'Recent Developments in the Field of Powder Coatings'

09.30-10.10 Dr. H. Liertz, Siemens AG, München, FRG

'The Influence of Coatings on the Fracture Behaviour of Light WaveGuides'

\subsection{0-10.45 Coffee break}

Filled Polymer-Systems

10.45-11.45 Dr. T. Kaiser, Asea Brown Boveri, Research Center, Baden, Switzerland 'Role of Particle-Matrix Interface in the Deformation and Fracture Behaviour of Filled Epoxy Resins

11.45-12.25 Dr. H.P. Schlumpf, Plüss-Staufer AG, Oftringen, Switzerland

'Physico-Chemical Aspects of Filers in Polypropylene'

12.25-13.30 Business Lunch

Composites

13.30-14.30 Dr. R.F. Siegmund, Ciba-Geigy Corporation, Composite Materials Department, Anaheim, USA

'Some Newer Applications of Advanced Polymer Composites'

14.30-15.10 Prof. A. Pavan, Dipartimento di Chimica Industriale e Ingegneria Chimica, Politecnico di Milano, Milano, Italy "A Study on the Toughening of Polymer Matrix Composites by Interphase Modification'

\section{Registration}

The registration card should be sent to: Secretary's Office for SAC-Symposia, Institute of Organic Chemistry, University of Berne, Freiestrasse 3, CH-3012 Berne, Switzerland, Tel. 031654311 , Telex 912406 uni Mixer at the Hotel Victoria Jungfrau ch, Fax 031 654499.

\section{Schweizerischer Wissenschaftsrat, Sekretariat Conseil suisse de la science, Secrétariat Consiglio svizzero della scienza, Segreteria}

\section{Forschung und Zukunft}

Empfehlungen des Schweizerischen Wissenschaftsrates für die Periode 19921995

Schwerpunkte auf den Forschungsgebieten Umwelt, soziopolitische Probleme, Technologie, eine konkurrenzfähigere Forschung auf internationaler Ebene; quantifizierte Vorschläge; Optionen im Bereich Forschungsmanagement: so lassen sich die Empfehlungen des Schweizerischen Wissenschaftsrates (SWR) für di Zukunft kurz umreissen.

Der Schweizerische Wissenschaftsrat hat soeben seine Empfehlungen für die schweizerische Forschungspolitik veröffentlicht. Diese dienten als Grundlage für die 'Ziele', die der Bundesrat vor kurzem angenommen hat. Fundierte Analysen und ein reichhaltiges Material sind Teil der Empfehlungen (Forschungsplatz Schweiz. Horizont 1995, SWR 1989, Bern 1990).

-- Umwelt/soziopolitische Probleme/Technologie: dre prioritäre Forschungsbereiche, die aber nicht auf Kosten der Grundlagenforschung bearbeitet werden sollen.

Der SWR unterstreicht vor allem folgende Forschungsbereiche, die in unserem Land noch ungenügend entwickelt sind: 1 . Schutz der Umwelt. 2. Bewältigung soziopolitischer Probleme wie Überalterung, Bitdung, Gesundheit (insbesondere in Bezug auf AIDS) 3. Neue Technologien und ihre Auswirkungen. Gleichzeitig hebt er hervor, dass die Grundlagenforschung der Grundstein jeglicher Aktivität im Bereich Forschung und Entwicklung $(\mathrm{F}+\mathrm{E})$ bleibt.

- Konkurrenzfähige Forschung auf internationaler Ebene

Der Bund muss die Bemühungen auf dem Gebiete von $F+E$ unterstützen. Im Hinblick auf das zukünftige Europa ist es sogar eine seiner Hauptaufgaben, die Forschung nicht nur für die eigenen Bedürfnisse zu fördern, sondern auch, um auf internationaler Ebene eine starke Position zu behalten.

Zahlen und konkrete Vorschläge der SWR

Der SWR hatte den Mut, die zusätzlichen Forschungsbedürfnisse für die Periode 1992-1995 in Zahlen zu nennen. So muss der Bund gewillt sein, seine Ausgaben für die Forschung, die sich bis anhin auf 1,5 Mia. Fr. pro Jahr beliefen (Nationalfonds, Hochschulen, Forschung im Rahmen der Bundesämter, internationale Engagements etc.) um 8 bis $10 \%$ pro Jah zu erhöhen. Momentan wird weit weniger als ein Viertel der Forschung von der öffentlichen Hand finanziert, der Rest wird von der Privatwirtschaft übernommen. Dieser Anteil ist einer der niedrigsten im internationalen Vergleich.

- Wie soll die Forschung verwaltet werden: freie Forschung vs. orientierte Forschung

Der SWR hatte ebenfalls den Mut, eine klare Grenzlinie zu ziehen zwischen der vom Staat orientierten Forschung, die auf die Bedürfnisse von Wirtschaft und Gesellschaft eingehen muss, und der Forschung, die frei bleiben muss, um ihrer Hauptaufgabe, neue Er- kenntnisse zu erbringen, gerecht zu werden. Mit einem besseren Forschungsmanagement sollen beide Forschungstypen verbessert werden: Planung, Koordination, Internationale Zusammenarbeit, Zusammenarbeit zwischen öffentlicher und privater Forschung, frühzeitiges Erkennen möglicher Forschungsbereiche, Evaluation der Resultate und Konsequenzen der Forschung und deren technischen Anwendungen.

Quelle: Forschungsplatz Schweiz. Horizont 1995, SWR 1989, Bern 1990.

Für weitere Auskünfte: Dr. François Da Pozzo Dr. Edo Poglia

Sekretariat des Schweizerischen Wissenschaftsrates Wildhainweg 9, 3001 Bern Telephon: 031619666

\section{IUPAC-Empfehlungen}

Nomenclature, symbols, units and their usage in spectrochemical analysis - XII. Terms related to electrothermal atomization

Terminology for electrothermal atomization is recommended and its use illustrated mainly in the application of electrothermal atomization to atomic absorption spectrometry. The terms, symbols and abbreviations required for the description of atomizers, for the processes involved in electrothermal atomization and in their analytical use are prescribed. An index of terms is included.

Comments on the document should be sent by Junc 30, 1991 to: Dr. T. A. M. Ure, Department of Pure and Applied Chemistry, Thomas Graham Building, University of Strathclyde, 295 Cathedral Street, Glasgow GI 1XL, UK. 


\section{DECHEMA-Weiterbildungskurs: Formulierungstechnik}

IBB Muttenz, 6.-9. November 1990

In einem viertägigen Kurs sollen die wissenschaftlich-technischen Grundlagen der Formulierungstechnik in einer allgemeinen, ganzheitlichen Sicht dargestellt werden. Diese Grundlagen entstammen vor allem der Kolloid- und Grenzflächenchemie und der Verfahrenstechnik. Sie erlauben die Darstellung der Formulierungstechnik, losgelöst von der stofflichen Seite, sowohl für Agrochemikalien, Farben, Lebensmittel, Waschmittel, Kosmetika, Feinchemikalien, Mineralien usw. Neben der eigentlichen Formulierung der betreffenden Stoffe mit den erforderlichen Zusatz- und Hilfsstoffen werden auch die technischen Einrichtungen zu ihrer Herstellung und Messmethoden zu ihrer physikalischen Charaktcrisicrung behandelt.

Die Formulierung von chemischen Stoffen aller Ar zu optimalen Handelsprodukten erfordert viclfältige Kenntnisse über die relevanten kolloidalen Systeme und die benötigten Hilfsmittel (z. B. Netzmittel, Dispergiermittel, allgemeine Tenside, Verdickungsmitte usw.) sowie über die technischen und apparativen Voraussetzungen ihrer Herstellung. Ohne diese Kenntnisse wird sich dic Formulierungstechnik auf eine rein empi- rische und weniger effiziente Bearbcitung reduzieren Das für den Formulierungschemiker wesentliche Grundwissen, insbesondere der Kolloid- und Grenzflächenwissenschaft, ist in der Literatur breit verstreu und oft nur schwer zugänglich. Mit Ausnahme der Galenik wurde bisher das Gebiet der Formulierungstechnik nicht in einer ganzheitlichen Betrachtung dargestellt. Diese Aufgabe wird mit diesem Kurs angestrebt und das Grundwissen dem Praktiker in anwendungsbezogener Form vermittelt. Dabei liegen die Schwerpunkte beispielsweisc auf: Dispergierung, Emulgierung, Solubilisation, Koazervation, Flockung, Agglomeration, Instantisierung. Ferner werden die entsprechenden Methoden der Teilchencharakterisierung behandelt.

Die einzelnen Sachgebiete werden von Fachleuten aus Industrie und Hochschule präsentiert. Eine Exkursion ergänzt das Programm.

Der Kurs wendet sich an Chemiker, Chemie-Ingenieure und Verfahrenstechniker in Produktion, Forschung und Entwicklung, die sich mit Formulierungsaufgaben beschäftigen müssen.

Information: Dr. D. Jahn/Dr. H. Mollet Ingenieurschule beider Basel (HTL)

$\mathrm{CH}-4312$ Muttenz

Tel.: (061)61 4242

\section{EUCHEM Conference}

\section{on Stereochemistry, Bürgenstock}

The 26th EUCHEM Conference on Stereochemistry, covering a wide range of topics in chemistry with emphasis on its interdisciplinary character in natura sciences, will be held at the Bürgenstock near Luzern, Switzerland from April 28-May 4, 1991.

Inquiries and applications (no special forms are required) should be addressed before January 10, 1991, to the President:

Professor H. Ringsdorf Institute of Organic Chemistry Johannes Gutenberg University J.-J.-Becher-Weg 1820

D-6500 Mainz

\section{Schweizerische Gesellschaft für Instrumentalanalytik und Mikrochemie Basel}

Dieses Schreiben soll über einige aktuelle Veranstaltungen auf dem Gebiet der analytischen Chemie informieren. Zudem möchte ich über den aktuellen Stand der Neustrukturierung der schweizerischen Vereinigungen auf dem Gebiet der Chemie orientieren.

Die Fusionsvorbereitungen zwischen der Schweizerischen Chemischen Gesellschaft und dem Schweizerischen Chemikerverband kommen gut voran, die konstituierende Versammlung einer neuen, gemeinsamen Gesellschaft ist für 1992 vorgesehen. Um in diesem Zusammenhang die Stellung der Analytischen Chemie zu stärken, wurde von einer Gruppe von Exponenten aus der schweizerischen analytischen Chemie das $\mathrm{Ko}$ mitee für Analytische Chemie Schweiz/Comité Suisse de Chimie Analytique (CSCA) gegründet. Als erster Präsident amtet Prof. H. M. Widmer, Ciba-Geigy AG, Basel. Aus dem Kreis der SGIM nehmen Dr. Heinzer und Prof. Haerdi als Beobachter an den Sitzungen des CSCA teil.

Als erste konkrete Aktion des CSCA wird gemein sam mit der Herbstversammlung der Schwcizerischen Chemischen Gesellschaft am 19. Oktober 1990 cin Analytikseminar durchgeführt. Falls es der Zeitplan dieses Seminars zulässt, planen wir gleichentags die Durchführung der jährlichen Geschäftssitzung de SGIM ein.

Dr. Franz Heinzer, Präsident SGIM 20. Mai 1990

\section{Comité Suisse de Chimie Analytique} (CSCA)

Am 19. Oktober 1990 findet in Bern die Herbstversammlung der Schweizerischen Chemischen Gesellschaft (SCG) statt, und wie Sie wissen, planen wir, dort ein ganztätiges Analytikseminar durchzuführen.

Dieser Anlass soll zum Aushängeschild der schweizerischen analytischen Chemie werden, und wir möchten höchste Ansprüche stellen. In den Vorträgen, zu denen wir die Redner einladen, sollen erfahrene Wissenschaftler des mittleren und oberen Kaders zu Worte kommen.

Wir gedenken, an zwei Plenarvorträgen Experten aus Übersee zu engagieren, und die übrige Zeit steht für rund zehn Vorträge von 20 Minuten Dauer zur Verfügung. Die von uns gewählten Themen beziehen sich au massenspektroskopische, chromatographische Titel arbeitshygienische Untersuchungen, Umweltanalytik, Sensortechnologie, sowie klinische Chemie, Thermoanalytik und Totale Analysensysteme für On-line-Analytik.

Neben diesen Vorträgen werden wir Poster zeigen können und auch hier streben wir ein ambitiöses Zie an: 30-50 Poster mit grösstmöglicher Themenfreiheit, aber die analytischen Wissenschaften betreffend.

Prof. Dr. H. M. Widmer, Präsident CSCA
March, 1991: Second Circular with preliminary programm

May, 1991: Deadline for registration
Authors working in this area are invited to submit an abstract of approx. 200 to 500 words until October 31 990 to the secretariat of HPLC '91, Convention Cen. ter Basel, Congress Department, P.O. Box, CH-402 Bascl (FAX: 061/691 8049).

Authors will be notified in January 1991. 


\section{DECHEMA-Weiterbildungskurs: Formulierungstechnik}

IBB Muttenz, 6.-9. November 1990

In einem viertägigen Kurs sollen die wissenschaftlich-technischen Grundlagen der Formulierungstechnik in einer allgemeinen, ganzheitlichen Sicht dargestellt werden. Diese Grundlagen entstammen vor allem der Kolloid- und Grenzflächenchemie und der Verfahrenstechnik. Sie erlauben die Darstellung der Formulierungstechnik, losgelöst von der stofflichen Seite, sowohl für Agrochemikalien, Farben, Lebensmittel, Waschmittel, Kosmetika, Feinchemikalien, Mineralien usw. Neben der eigentlichen Formulierung der betreffenden Stoffe mit den erforderlichen Zusatz- und Hilfsstoffen werden auch die technischen Einrichtungen zu ihrer Herstellung und Messmethoden zu ihrer physikalischen Charaktcrisicrung behandelt.

Die Formulierung von chemischen Stoffen aller Ar zu optimalen Handelsprodukten erfordert viclfältige Kenntnisse über die relevanten kolloidalen Systeme und die benötigten Hilfsmittel (z. B. Netzmittel, Dispergiermittel, allgemeine Tenside, Verdickungsmitte usw.) sowie über die technischen und apparativen Voraussetzungen ihrer Herstellung. Ohne diese Kenntnisse wird sich dic Formulierungstechnik auf eine rein empi- rische und weniger effiziente Bearbcitung reduzieren Das für den Formulierungschemiker wesentliche Grundwissen, insbesondere der Kolloid- und Grenzflächenwissenschaft, ist in der Literatur breit verstreu und oft nur schwer zugänglich. Mit Ausnahme der Galenik wurde bisher das Gebiet der Formulierungstechnik nicht in einer ganzheitlichen Betrachtung dargestellt. Diese Aufgabe wird mit diesem Kurs angestrebt und das Grundwissen dem Praktiker in anwendungsbezogener Form vermittelt. Dabei liegen die Schwerpunkte beispielsweisc auf: Dispergierung, Emulgierung, Solubilisation, Koazervation, Flockung, Agglomeration, Instantisierung. Ferner werden die entsprechenden Methoden der Teilchencharakterisierung behandelt.

Die einzelnen Sachgebiete werden von Fachleuten aus Industrie und Hochschule präsentiert. Eine Exkursion ergänzt das Programm.

Der Kurs wendet sich an Chemiker, Chemie-Ingenieure und Verfahrenstechniker in Produktion, Forschung und Entwicklung, die sich mit Formulierungsaufgaben beschäftigen müssen.

Information: Dr. D. Jahn/Dr. H. Mollet Ingenieurschule beider Basel (HTL)

$\mathrm{CH}-4312$ Muttenz

Tel.: (061)61 4242

\section{EUCHEM Conference}

\section{on Stereochemistry, Bürgenstock}

The 26th EUCHEM Conference on Stereochemistry, covering a wide range of topics in chemistry with emphasis on its interdisciplinary character in natura sciences, will be held at the Bürgenstock near Luzern, Switzerland from April 28-May 4, 1991.

Inquiries and applications (no special forms are required) should be addressed before January 10, 1991, to the President:

Professor H. Ringsdorf Institute of Organic Chemistry Johannes Gutenberg University J.-J.-Becher-Weg 1820

D-6500 Mainz

\section{Schweizerische Gesellschaft für Instrumentalanalytik und Mikrochemie Basel}

Dieses Schreiben soll über einige aktuelle Veranstaltungen auf dem Gebiet der analytischen Chemie informieren. Zudem möchte ich über den aktuellen Stand der Neustrukturierung der schweizerischen Vereinigungen auf dem Gebiet der Chemie orientieren.

Die Fusionsvorbereitungen zwischen der Schweizerischen Chemischen Gesellschaft und dem Schweizerischen Chemikerverband kommen gut voran, die konstituierende Versammlung einer neuen, gemeinsamen Gesellschaft ist für 1992 vorgesehen. Um in diesem Zusammenhang die Stellung der Analytischen Chemie zu stärken, wurde von einer Gruppe von Exponenten aus der schweizerischen analytischen Chemie das $\mathrm{Ko}$ mitee für Analytische Chemie Schweiz/Comité Suisse de Chimie Analytique (CSCA) gegründet. Als erster Präsident amtet Prof. H. M. Widmer, Ciba-Geigy AG, Basel. Aus dem Kreis der SGIM nehmen Dr. Heinzer und Prof. Haerdi als Beobachter an den Sitzungen des CSCA teil.

Als erste konkrete Aktion des CSCA wird gemein sam mit der Herbstversammlung der Schwcizerischen Chemischen Gesellschaft am 19. Oktober 1990 cin Analytikseminar durchgeführt. Falls es der Zeitplan dieses Seminars zulässt, planen wir gleichentags die Durchführung der jährlichen Geschäftssitzung de SGIM ein.

Dr. Franz Heinzer, Präsident SGIM 20. Mai 1990

\section{Comité Suisse de Chimie Analytique} (CSCA)

Am 19. Oktober 1990 findet in Bern die Herbstversammlung der Schweizerischen Chemischen Gesellschaft (SCG) statt, und wie Sie wissen, planen wir, dort ein ganztätiges Analytikseminar durchzuführen.

Dieser Anlass soll zum Aushängeschild der schweizerischen analytischen Chemie werden, und wir möchten höchste Ansprüche stellen. In den Vorträgen, zu denen wir die Redner einladen, sollen erfahrene Wissenschaftler des mittleren und oberen Kaders zu Worte kommen.

Wir gedenken, an zwei Plenarvorträgen Experten aus Übersee zu engagieren, und die übrige Zeit steht für rund zehn Vorträge von 20 Minuten Dauer zur Verfügung. Die von uns gewählten Themen beziehen sich au massenspektroskopische, chromatographische Titel arbeitshygienische Untersuchungen, Umweltanalytik, Sensortechnologie, sowie klinische Chemie, Thermoanalytik und Totale Analysensysteme für On-line-Analytik.

Neben diesen Vorträgen werden wir Poster zeigen können und auch hier streben wir ein ambitiöses Zie an: 30-50 Poster mit grösstmöglicher Themenfreiheit, aber die analytischen Wissenschaften betreffend.

Prof. Dr. H. M. Widmer, Präsident CSCA
March, 1991: Second Circular with preliminary programm

May, 1991: Deadline for registration
Authors working in this area are invited to submit an abstract of approx. 200 to 500 words until October 31 990 to the secretariat of HPLC '91, Convention Cen. ter Basel, Congress Department, P.O. Box, CH-402 Bascl (FAX: 061/691 8049).

Authors will be notified in January 1991. 


\section{DECHEMA-Weiterbildungskurs: Formulierungstechnik}

IBB Muttenz, 6.-9. November 1990

In einem viertägigen Kurs sollen die wissenschaftlich-technischen Grundlagen der Formulierungstechnik in einer allgemeinen, ganzheitlichen Sicht dargestellt werden. Diese Grundlagen entstammen vor allem der Kolloid- und Grenzflächenchemie und der Verfahrenstechnik. Sie erlauben die Darstellung der Formulierungstechnik, losgelöst von der stofflichen Seite, sowohl für Agrochemikalien, Farben, Lebensmittel, Waschmittel, Kosmetika, Feinchemikalien, Mineralien usw. Neben der eigentlichen Formulierung der betreffenden Stoffe mit den erforderlichen Zusatz- und Hilfsstoffen werden auch die technischen Einrichtungen zu ihrer Herstellung und Messmethoden zu ihrer physikalischen Charaktcrisicrung behandelt.

Die Formulierung von chemischen Stoffen aller Ar zu optimalen Handelsprodukten erfordert viclfältige Kenntnisse über die relevanten kolloidalen Systeme und die benötigten Hilfsmittel (z. B. Netzmittel, Dispergiermittel, allgemeine Tenside, Verdickungsmitte usw.) sowie über die technischen und apparativen Voraussetzungen ihrer Herstellung. Ohne diese Kenntnisse wird sich dic Formulierungstechnik auf eine rein empi- rische und weniger effiziente Bearbcitung reduzieren Das für den Formulierungschemiker wesentliche Grundwissen, insbesondere der Kolloid- und Grenzflächenwissenschaft, ist in der Literatur breit verstreu und oft nur schwer zugänglich. Mit Ausnahme der Galenik wurde bisher das Gebiet der Formulierungstechnik nicht in einer ganzheitlichen Betrachtung dargestellt. Diese Aufgabe wird mit diesem Kurs angestrebt und das Grundwissen dem Praktiker in anwendungsbezogener Form vermittelt. Dabei liegen die Schwerpunkte beispielsweisc auf: Dispergierung, Emulgierung, Solubilisation, Koazervation, Flockung, Agglomeration, Instantisierung. Ferner werden die entsprechenden Methoden der Teilchencharakterisierung behandelt.

Die einzelnen Sachgebiete werden von Fachleuten aus Industrie und Hochschule präsentiert. Eine Exkursion ergänzt das Programm.

Der Kurs wendet sich an Chemiker, Chemie-Ingenieure und Verfahrenstechniker in Produktion, Forschung und Entwicklung, die sich mit Formulierungsaufgaben beschäftigen müssen.

Information: Dr. D. Jahn/Dr. H. Mollet Ingenieurschule beider Basel (HTL)

$\mathrm{CH}-4312$ Muttenz

Tel.: (061)61 4242

\section{EUCHEM Conference}

\section{on Stereochemistry, Bürgenstock}

The 26th EUCHEM Conference on Stereochemistry, covering a wide range of topics in chemistry with emphasis on its interdisciplinary character in natura sciences, will be held at the Bürgenstock near Luzern, Switzerland from April 28-May 4, 1991.

Inquiries and applications (no special forms are required) should be addressed before January 10, 1991, to the President:

Professor H. Ringsdorf Institute of Organic Chemistry Johannes Gutenberg University J.-J.-Becher-Weg 1820

D-6500 Mainz

\section{Schweizerische Gesellschaft für Instrumentalanalytik und Mikrochemie Basel}

Dieses Schreiben soll über einige aktuelle Veranstaltungen auf dem Gebiet der analytischen Chemie informieren. Zudem möchte ich über den aktuellen Stand der Neustrukturierung der schweizerischen Vereinigungen auf dem Gebiet der Chemie orientieren.

Die Fusionsvorbereitungen zwischen der Schweizerischen Chemischen Gesellschaft und dem Schweizerischen Chemikerverband kommen gut voran, die konstituierende Versammlung einer neuen, gemeinsamen Gesellschaft ist für 1992 vorgesehen. Um in diesem Zusammenhang die Stellung der Analytischen Chemie zu stärken, wurde von einer Gruppe von Exponenten aus der schweizerischen analytischen Chemie das $\mathrm{Ko}$ mitee für Analytische Chemie Schweiz/Comité Suisse de Chimie Analytique (CSCA) gegründet. Als erster Präsident amtet Prof. H. M. Widmer, Ciba-Geigy AG, Basel. Aus dem Kreis der SGIM nehmen Dr. Heinzer und Prof. Haerdi als Beobachter an den Sitzungen des CSCA teil.

Als erste konkrete Aktion des CSCA wird gemein sam mit der Herbstversammlung der Schwcizerischen Chemischen Gesellschaft am 19. Oktober 1990 cin Analytikseminar durchgeführt. Falls es der Zeitplan dieses Seminars zulässt, planen wir gleichentags die Durchführung der jährlichen Geschäftssitzung de SGIM ein.

Dr. Franz Heinzer, Präsident SGIM 20. Mai 1990

\section{Comité Suisse de Chimie Analytique} (CSCA)

Am 19. Oktober 1990 findet in Bern die Herbstversammlung der Schweizerischen Chemischen Gesellschaft (SCG) statt, und wie Sie wissen, planen wir, dort ein ganztätiges Analytikseminar durchzuführen.

Dieser Anlass soll zum Aushängeschild der schweizerischen analytischen Chemie werden, und wir möchten höchste Ansprüche stellen. In den Vorträgen, zu denen wir die Redner einladen, sollen erfahrene Wissenschaftler des mittleren und oberen Kaders zu Worte kommen.

Wir gedenken, an zwei Plenarvorträgen Experten aus Übersee zu engagieren, und die übrige Zeit steht für rund zehn Vorträge von 20 Minuten Dauer zur Verfügung. Die von uns gewählten Themen beziehen sich au massenspektroskopische, chromatographische Titel arbeitshygienische Untersuchungen, Umweltanalytik, Sensortechnologie, sowie klinische Chemie, Thermoanalytik und Totale Analysensysteme für On-line-Analytik.

Neben diesen Vorträgen werden wir Poster zeigen können und auch hier streben wir ein ambitiöses Zie an: 30-50 Poster mit grösstmöglicher Themenfreiheit, aber die analytischen Wissenschaften betreffend.

Prof. Dr. H. M. Widmer, Präsident CSCA
March, 1991: Second Circular with preliminary programm

May, 1991: Deadline for registration
Authors working in this area are invited to submit an abstract of approx. 200 to 500 words until October 31 990 to the secretariat of HPLC '91, Convention Cen. ter Basel, Congress Department, P.O. Box, CH-402 Bascl (FAX: 061/691 8049).

Authors will be notified in January 1991. 


\section{DECHEMA-Weiterbildungskurs: Formulierungstechnik}

IBB Muttenz, 6.-9. November 1990

In einem viertägigen Kurs sollen die wissenschaftlich-technischen Grundlagen der Formulierungstechnik in einer allgemeinen, ganzheitlichen Sicht dargestellt werden. Diese Grundlagen entstammen vor allem der Kolloid- und Grenzflächenchemie und der Verfahrenstechnik. Sie erlauben die Darstellung der Formulierungstechnik, losgelöst von der stofflichen Seite, sowohl für Agrochemikalien, Farben, Lebensmittel, Waschmittel, Kosmetika, Feinchemikalien, Mineralien usw. Neben der eigentlichen Formulierung der betreffenden Stoffe mit den erforderlichen Zusatz- und Hilfsstoffen werden auch die technischen Einrichtungen zu ihrer Herstellung und Messmethoden zu ihrer physikalischen Charaktcrisicrung behandelt.

Die Formulierung von chemischen Stoffen aller Ar zu optimalen Handelsprodukten erfordert viclfältige Kenntnisse über die relevanten kolloidalen Systeme und die benötigten Hilfsmittel (z. B. Netzmittel, Dispergiermittel, allgemeine Tenside, Verdickungsmitte usw.) sowie über die technischen und apparativen Voraussetzungen ihrer Herstellung. Ohne diese Kenntnisse wird sich dic Formulierungstechnik auf eine rein empi- rische und weniger effiziente Bearbcitung reduzieren Das für den Formulierungschemiker wesentliche Grundwissen, insbesondere der Kolloid- und Grenzflächenwissenschaft, ist in der Literatur breit verstreu und oft nur schwer zugänglich. Mit Ausnahme der Galenik wurde bisher das Gebiet der Formulierungstechnik nicht in einer ganzheitlichen Betrachtung dargestellt. Diese Aufgabe wird mit diesem Kurs angestrebt und das Grundwissen dem Praktiker in anwendungsbezogener Form vermittelt. Dabei liegen die Schwerpunkte beispielsweisc auf: Dispergierung, Emulgierung, Solubilisation, Koazervation, Flockung, Agglomeration, Instantisierung. Ferner werden die entsprechenden Methoden der Teilchencharakterisierung behandelt.

Die einzelnen Sachgebiete werden von Fachleuten aus Industrie und Hochschule präsentiert. Eine Exkursion ergänzt das Programm.

Der Kurs wendet sich an Chemiker, Chemie-Ingenieure und Verfahrenstechniker in Produktion, Forschung und Entwicklung, die sich mit Formulierungsaufgaben beschäftigen müssen.

Information: Dr. D. Jahn/Dr. H. Mollet Ingenieurschule beider Basel (HTL)

$\mathrm{CH}-4312$ Muttenz

Tel.: (061)61 4242

\section{EUCHEM Conference}

\section{on Stereochemistry, Bürgenstock}

The 26th EUCHEM Conference on Stereochemistry, covering a wide range of topics in chemistry with emphasis on its interdisciplinary character in natura sciences, will be held at the Bürgenstock near Luzern, Switzerland from April 28-May 4, 1991.

Inquiries and applications (no special forms are required) should be addressed before January 10, 1991, to the President:

Professor H. Ringsdorf Institute of Organic Chemistry Johannes Gutenberg University J.-J.-Becher-Weg 1820

D-6500 Mainz

\section{Schweizerische Gesellschaft für Instrumentalanalytik und Mikrochemie Basel}

Dieses Schreiben soll über einige aktuelle Veranstaltungen auf dem Gebiet der analytischen Chemie informieren. Zudem möchte ich über den aktuellen Stand der Neustrukturierung der schweizerischen Vereinigungen auf dem Gebiet der Chemie orientieren.

Die Fusionsvorbereitungen zwischen der Schweizerischen Chemischen Gesellschaft und dem Schweizerischen Chemikerverband kommen gut voran, die konstituierende Versammlung einer neuen, gemeinsamen Gesellschaft ist für 1992 vorgesehen. Um in diesem Zusammenhang die Stellung der Analytischen Chemie zu stärken, wurde von einer Gruppe von Exponenten aus der schweizerischen analytischen Chemie das $\mathrm{Ko}$ mitee für Analytische Chemie Schweiz/Comité Suisse de Chimie Analytique (CSCA) gegründet. Als erster Präsident amtet Prof. H. M. Widmer, Ciba-Geigy AG, Basel. Aus dem Kreis der SGIM nehmen Dr. Heinzer und Prof. Haerdi als Beobachter an den Sitzungen des CSCA teil.

Als erste konkrete Aktion des CSCA wird gemein sam mit der Herbstversammlung der Schwcizerischen Chemischen Gesellschaft am 19. Oktober 1990 cin Analytikseminar durchgeführt. Falls es der Zeitplan dieses Seminars zulässt, planen wir gleichentags die Durchführung der jährlichen Geschäftssitzung de SGIM ein.

Dr. Franz Heinzer, Präsident SGIM 20. Mai 1990

\section{Comité Suisse de Chimie Analytique} (CSCA)

Am 19. Oktober 1990 findet in Bern die Herbstversammlung der Schweizerischen Chemischen Gesellschaft (SCG) statt, und wie Sie wissen, planen wir, dort ein ganztätiges Analytikseminar durchzuführen.

Dieser Anlass soll zum Aushängeschild der schweizerischen analytischen Chemie werden, und wir möchten höchste Ansprüche stellen. In den Vorträgen, zu denen wir die Redner einladen, sollen erfahrene Wissenschaftler des mittleren und oberen Kaders zu Worte kommen.

Wir gedenken, an zwei Plenarvorträgen Experten aus Übersee zu engagieren, und die übrige Zeit steht für rund zehn Vorträge von 20 Minuten Dauer zur Verfügung. Die von uns gewählten Themen beziehen sich au massenspektroskopische, chromatographische Titel arbeitshygienische Untersuchungen, Umweltanalytik, Sensortechnologie, sowie klinische Chemie, Thermoanalytik und Totale Analysensysteme für On-line-Analytik.

Neben diesen Vorträgen werden wir Poster zeigen können und auch hier streben wir ein ambitiöses Zie an: 30-50 Poster mit grösstmöglicher Themenfreiheit, aber die analytischen Wissenschaften betreffend.

Prof. Dr. H. M. Widmer, Präsident CSCA
March, 1991: Second Circular with preliminary programm

May, 1991: Deadline for registration
Authors working in this area are invited to submit an abstract of approx. 200 to 500 words until October 31 990 to the secretariat of HPLC '91, Convention Cen. ter Basel, Congress Department, P.O. Box, CH-402 Bascl (FAX: 061/691 8049).

Authors will be notified in January 1991. 


\section{DECHEMA-Weiterbildungskurs: Formulierungstechnik}

IBB Muttenz, 6.-9. November 1990

In einem viertägigen Kurs sollen die wissenschaftlich-technischen Grundlagen der Formulierungstechnik in einer allgemeinen, ganzheitlichen Sicht dargestellt werden. Diese Grundlagen entstammen vor allem der Kolloid- und Grenzflächenchemie und der Verfahrenstechnik. Sie erlauben die Darstellung der Formulierungstechnik, losgelöst von der stofflichen Seite, sowohl für Agrochemikalien, Farben, Lebensmittel, Waschmittel, Kosmetika, Feinchemikalien, Mineralien usw. Neben der eigentlichen Formulierung der betreffenden Stoffe mit den erforderlichen Zusatz- und Hilfsstoffen werden auch die technischen Einrichtungen zu ihrer Herstellung und Messmethoden zu ihrer physikalischen Charaktcrisicrung behandelt.

Die Formulierung von chemischen Stoffen aller Ar zu optimalen Handelsprodukten erfordert viclfältige Kenntnisse über die relevanten kolloidalen Systeme und die benötigten Hilfsmittel (z. B. Netzmittel, Dispergiermittel, allgemeine Tenside, Verdickungsmitte usw.) sowie über die technischen und apparativen Voraussetzungen ihrer Herstellung. Ohne diese Kenntnisse wird sich dic Formulierungstechnik auf eine rein empi- rische und weniger effiziente Bearbcitung reduzieren Das für den Formulierungschemiker wesentliche Grundwissen, insbesondere der Kolloid- und Grenzflächenwissenschaft, ist in der Literatur breit verstreu und oft nur schwer zugänglich. Mit Ausnahme der Galenik wurde bisher das Gebiet der Formulierungstechnik nicht in einer ganzheitlichen Betrachtung dargestellt. Diese Aufgabe wird mit diesem Kurs angestrebt und das Grundwissen dem Praktiker in anwendungsbezogener Form vermittelt. Dabei liegen die Schwerpunkte beispielsweisc auf: Dispergierung, Emulgierung, Solubilisation, Koazervation, Flockung, Agglomeration, Instantisierung. Ferner werden die entsprechenden Methoden der Teilchencharakterisierung behandelt.

Die einzelnen Sachgebiete werden von Fachleuten aus Industrie und Hochschule präsentiert. Eine Exkursion ergänzt das Programm.

Der Kurs wendet sich an Chemiker, Chemie-Ingenieure und Verfahrenstechniker in Produktion, Forschung und Entwicklung, die sich mit Formulierungsaufgaben beschäftigen müssen.

Information: Dr. D. Jahn/Dr. H. Mollet Ingenieurschule beider Basel (HTL)

$\mathrm{CH}-4312$ Muttenz

Tel.: (061)61 4242

\section{EUCHEM Conference}

\section{on Stereochemistry, Bürgenstock}

The 26th EUCHEM Conference on Stereochemistry, covering a wide range of topics in chemistry with emphasis on its interdisciplinary character in natura sciences, will be held at the Bürgenstock near Luzern, Switzerland from April 28-May 4, 1991.

Inquiries and applications (no special forms are required) should be addressed before January 10, 1991, to the President:

Professor H. Ringsdorf Institute of Organic Chemistry Johannes Gutenberg University J.-J.-Becher-Weg 1820

D-6500 Mainz

\section{Schweizerische Gesellschaft für Instrumentalanalytik und Mikrochemie Basel}

Dieses Schreiben soll über einige aktuelle Veranstaltungen auf dem Gebiet der analytischen Chemie informieren. Zudem möchte ich über den aktuellen Stand der Neustrukturierung der schweizerischen Vereinigungen auf dem Gebiet der Chemie orientieren.

Die Fusionsvorbereitungen zwischen der Schweizerischen Chemischen Gesellschaft und dem Schweizerischen Chemikerverband kommen gut voran, die konstituierende Versammlung einer neuen, gemeinsamen Gesellschaft ist für 1992 vorgesehen. Um in diesem Zusammenhang die Stellung der Analytischen Chemie zu stärken, wurde von einer Gruppe von Exponenten aus der schweizerischen analytischen Chemie das $\mathrm{Ko}$ mitee für Analytische Chemie Schweiz/Comité Suisse de Chimie Analytique (CSCA) gegründet. Als erster Präsident amtet Prof. H. M. Widmer, Ciba-Geigy AG, Basel. Aus dem Kreis der SGIM nehmen Dr. Heinzer und Prof. Haerdi als Beobachter an den Sitzungen des CSCA teil.

Als erste konkrete Aktion des CSCA wird gemein sam mit der Herbstversammlung der Schwcizerischen Chemischen Gesellschaft am 19. Oktober 1990 cin Analytikseminar durchgeführt. Falls es der Zeitplan dieses Seminars zulässt, planen wir gleichentags die Durchführung der jährlichen Geschäftssitzung de SGIM ein.

Dr. Franz Heinzer, Präsident SGIM 20. Mai 1990

\section{Comité Suisse de Chimie Analytique} (CSCA)

Am 19. Oktober 1990 findet in Bern die Herbstversammlung der Schweizerischen Chemischen Gesellschaft (SCG) statt, und wie Sie wissen, planen wir, dort ein ganztätiges Analytikseminar durchzuführen.

Dieser Anlass soll zum Aushängeschild der schweizerischen analytischen Chemie werden, und wir möchten höchste Ansprüche stellen. In den Vorträgen, zu denen wir die Redner einladen, sollen erfahrene Wissenschaftler des mittleren und oberen Kaders zu Worte kommen.

Wir gedenken, an zwei Plenarvorträgen Experten aus Übersee zu engagieren, und die übrige Zeit steht für rund zehn Vorträge von 20 Minuten Dauer zur Verfügung. Die von uns gewählten Themen beziehen sich au massenspektroskopische, chromatographische Titel arbeitshygienische Untersuchungen, Umweltanalytik, Sensortechnologie, sowie klinische Chemie, Thermoanalytik und Totale Analysensysteme für On-line-Analytik.

Neben diesen Vorträgen werden wir Poster zeigen können und auch hier streben wir ein ambitiöses Zie an: 30-50 Poster mit grösstmöglicher Themenfreiheit, aber die analytischen Wissenschaften betreffend.

Prof. Dr. H. M. Widmer, Präsident CSCA
March, 1991: Second Circular with preliminary programm

May, 1991: Deadline for registration
Authors working in this area are invited to submit an abstract of approx. 200 to 500 words until October 31 990 to the secretariat of HPLC '91, Convention Cen. ter Basel, Congress Department, P.O. Box, CH-402 Bascl (FAX: 061/691 8049).

Authors will be notified in January 1991. 


\section{Personalia}

\section{Geburtstage}

Hans-Rudolf Schmid, Dr. sc. techn., Riehen, Mitglied des SChV, feiert am 16.9.90 seinen 65. Geburtstag.

Max J. Ruther, Dr. rer. nat., Forch, Mitglied des SChV, feiert an 20.9.90 seinen 70. Geburtstag.

\section{Neue Mitglieder}

Ács Mária, Prof. Dr., Dept. Org. Chem. Technology, TU Budapest, Postfach 91, H-1521 Budapest.

Breitschuh Richard, Dipl. Chem., Sprensenbühl 10 8032 Zürich

Von dem Bussche-H. Christoph, Dipl. Chem., Herbstweg 72, 8050 Zürich.

\section{Wahlen}

Als ordentlicher Professor für informatikgestützte Chemie an der ETH Zürich wurde auf den 1. September 1990 gewählt: Wilfred F. van Gunsteren, Ph. D. geboren 1947, niederländischer Staatsbürger, zurzeit Professor für physikalische Chemie an der Universität Groningen NL.

Als ordentlicher Professor für allgemeine und anorganische Chemie an der ETH Zürich wurde auf den 1.Oktober 1990 gewählt: Reinhard Nesper, Dr. rer nat., geboren 1949, deutscher Staatsangehöriger, zurzeit wissenschaftlicher Mitarbeiter am Max-Planck-Institut für Festkörperforschung in Stuttgart.

PD Dr. Urs Séquin wurde zu einem ausserordentlichen Professor der Philosophisch-Naturwissenschaftlichen Fakultät der Universität Basel ernannt.

\section{Gründung einer Fachgruppe Rheologie}

Am 24. April 1990 fand in der ETH Zürich eine In formationstagung Rheologie statt, die von der Polymer-Gruppe der Schweiz (PGS) organisiert worden ist. Diese Veranstaltung sollte die an der Rheologie Interessierten zusammenführen und zeigen, auf welchen $\mathrm{Ge}$ bieten in der Schweiz rheologische Aktivitäten beste. hen.

Es wurden folgende Vorträge gehalten:

Prof. Dr. F. Escher

Institut für Lebensmittelwissenschaft

ETH Zürich

Rheologie in der Lebensmitteltechnologie

Prof. Dr. J. Meissner

Institut für Polymere, ETH Zürich

Messverfahren in der Rheologie von Polymer-Schmelzen

Dr. D. Hadjistamov

Ciba-Geigy AG, 4133 Schweizerhalle

Rheologie von formulierten dispersen Systemen

Dr. A. Gyr

Institut für Hydromechanik und Wasserbau

ETH-Zürich

Rheologie in der Turbulenzforschung von Wasser

Dr. Th. Raible

Geberit AG, 8640 Rapperswil

Rheologische Auslegung von Spritzgusswerkzeugen

Am Ende der Informationstagung wurde von den Anwesenden einstimmig der Beschluss gefasst, eine Fachgruppe Rheologie innerhalb der Polymer-Gruppe der Schweiz zu bilden. Gleichzeitig wurden folgende vier Mitglieder in die Leitung der Fachgruppe Rheologie gewählt:

Prof. Dr. F. Escher, Institut für Lebensmittelwissen schaft, ETH Zürich

Dr. D. Hadjistamov, Ciba-Geigy AG, 4133 Schweizerhalle

Prof. Dr. J. Meissner, Institut für Polymere, ETH Zürich

Dr. Th. Raible, Geberit AG, 8640 Rapperswil

Zum Leiter der Fachgruppe Rheologie wurde am 15. Mai 1990 bei der Sitzung der Gruppenleitung Prof Dr. J. Meissner gewählt, Herr Prof. Dr. F. Escher zu seinem Stellvertreter. Die Amtsperiode beträgt jeweils zwei Jahre, Wiederwahl ist zulässig.

Die Leitung der Fachgruppe sieht folgende zukünftige Aktivitäten für die Fachgruppe Rheologie:

1. Ausbildung in Rheologie auf Anfängerniveau,

2. Fortbildung für Fortgeschrittene,

3. Behandlung wissenschaftlicher und anwendungsbezogener Themen sowie spezieller Forschungsfragen,

4. Informationsveranstaltungen über bestimmte Themen mit (wenn möglich) Demonstrationen,

5. lokale Tagungen über spezielle Themen,

6. Organisation von Exkursionen zu ausgewählten Firmen.

Zur Frage der Mitglicdschaft sei festgehalten, dass jedes Mitglied der Polymer-Gruppe der Schweiz ohne zusätzliche Kosten Mitglied der Fachgruppe Rheologie werden kann. Dementsprechend setzt die Mitgliedschaft in der Fachgruppe Rheologie die Mitgliedschaft in der PGS voraus.

Zürich, 12. Juni 1990

Prof. Dr. J. Meissner

\section{Biotechnologie für Chemiker}

Die Abteilung für Chemie am Technikum Winterthur Ingenieurschule (TWI) bietet ein einjähriges Nachdiplomstudium an:

Dieses Studium dauert zwei Semester à 19 Wochen mit je 30 Wochenstunden. Es wird zum zweiten Mal vom 12.11.1990 bis 12.10.1991 durchgeführt.

Ausbildungsziel ist die theoretische und praktische Einführung in die Biotechnologie und deren Anwendung in den Gebieten Bioverfahrenstechnik, Analytik und Ökologie

Der Kurs richtet sich an Chemiker, die sich für praktische Anwendungen biotechnischer Verfahren interessieren, sowie an weitere Interessenten mit geeigneter Vorbildung und Praxis.

Dic Teilnehmerzahl ist auf 12 beschränkt

Interessenten erhalten weitere Informationen* bei:

Technikum Winterthur Ingenieurschule

Prof. Dr. G. Wolf

Postfach

CH-8401 Winterthur

Telephon (052) 8263 23/64, Kanzlei: (052) 826161

Telefax (052) 826383

*Vgl. dazu G.Wolf, CHIMIA 1988, 42, 342; 1990, 44 33 .

\section{Erfolgreiche Basler Chemie-Konzerne}

I.C. - Die drei grössten Schweizer Chemieunternehmen Ciba-Geigy, Hoffmann-La Roche und Sandoz konnten an ihren Bilanzpressekonferenzen für das Jahr 1989 wiederum sehr gute Erfolge präsentieren. Auf den internationalen Märkten haben die drei Konzerne Umsätze in Höhe von fast $43 \mathrm{Mrd}$. Franken erreicht. Das sind rund $6 \mathrm{Mrd}$. Franken oder $17 \%$ mehr als im Vorjahr. $40 \%$ dieses Wachstums sind allerdings währungsbedingt. Insgesamt beschäftigten die drei Basler Konzerne weltweit 193411 Mitarbeiterinnen und Mitarbeiter, davon 51976 oder $27 \%$ in der Schweiz.

Grosse Mittel, nämlich rund $4 \mathrm{Mrd}$. Franken, wurden weltweit in Sachanlagen investiert. Davon entfielen rund 1,37 Mrd. Franken oder 34\% auf Neueinrichtungen in Schweizer Chemiewerken, was ein volkswirtschaftlich beachtliches Volumen für das Baugewerbe und die Ausrüstungsindustrie darstellt.
Die Forschungs- und Entwicklungsaufwendungen beliefen sich 1989 auf insgesamt 4,6 Mrd. Franken oder $10,7 \%$ der Umsätze. Wie Ciba-Geigy in ihrem Geschäftsbericht schreibt, findet das Wachstum bei $F+E$ vor allem im Ausland statt, da in der Schweiz der beschränkt vorhandene Chemikernachwuchs diesem Gebiet Grenzen setzt

Beachtlich sind die Aufwendungen der Basler Stammhäuser mit rund $750 \mathrm{Mio}$. Franken für die Sicherheit und den Umweltschutz. Alle Unternehmensführungen betrachten den Schutz der Umwelt neben dem wirtschaftichen Erfolg als Teil der unternehmerischen Gesamtverantwortung. Produktesicherheit, Sicherheit bei der Prozessabwicklung und Umweltschutz in der Produktion sind heute feste Beiträge zum qualitativen Wachstum der Branche Chemie.

\section{ALUSUISSE-LONZA übernimmt Cyclo in USA}

Basel, 17. Juli 1990 - Der Bereich Organische Chemie der ALUSUISSE-LONZA-Gruppe hat durch Alusuisse of America lnc. die Cyclo Products Inc. und die Schwestergesellschaft Balenco Enterprises Inc. Lo Angeles, USA, übernommen. Diese Unternehmen sind auf die Herstellung von hochwertigen Zwischenprodukten und Bulk Generika spezialisiert und beschäftigen 60 Mitarbeiter bei einem Umsatz von annähernd USS 11 Mio. Die Aktivitäten der akquirierten Unternehmen passen sehr gut in die bestehende Produktepalette der LONZA AG. Mit der Akquisition kann der Bereich Organische Chemie der ALUSUISSE LONZA-Gruppe sein Sortiment auf dem Gebiete der Feinchemikalien erweitern und seine Position im amerikanischen Markt verbessern. Für den Vertrieb ihrer Produkte können beide Unternehmen das weltweite Distributionsnetz der ALUSUISSE-LONZA-Gruppe benutzen.

\section{ILMAC 90 - die Chemiefachmesse in der Chemiemetropole}

Basel, als europäische Chemie- und Pharmaziemetropole, ist der geeignete Durchführungsort für die ILMAC 90, 11. Internationale Chemiefachmesse für Laboratoriums- und Verfahrenstechnik, Messtechnik und Automatik, vom 23. bis 26. Oktober 1990 in den Hallen der Schweizer Mustermesse.

Die Vergangenheit hat gezeigt, dass dem Risikaabbau in den Produktionsprozessen der chemischen Industrie ein immer höherer Stellenwert beizumessen ist. Innovative und v. a. umweltfreundlichere Lösungen in der Verfahrens-, Mess-, Regel- und Automationstechnik drängen sich auf.

Im Zuge dieser dynamischen Entwicklung bietet die ILMAC eine Standortbestimmung. Als eine der international wichtigsten Fachmessen auf ihrem Gebiet fasst sie alle Bereiche der chemischen Produktion zusammen: Laborausstattung und -technik; Isotopen und Radiochemie; Verfahrenstechnik; Mess-, Regelund Automationstechnik; Biotechnologie; Labortierhaltung; Umwelt- und Gesundheitsschutz; Laboreinrichtungen für Arztpraxen sowie Dienstleistungen. Durch die Erschliessung eines neuen Sektors, Laboreinrichtungen für Arztpraxen, werden an der ILMAC in vermehrtem Masse die Ärzte angesprochen. Bei diesen besteht ein wachsendes Bedürfnis für entsprechende Einrichtungen in den Praxen.

Die im Dreijahresturnus in Zusammenarbeit mit dem Schweizerischen Chemiker-Verband veranstaltete ILMAC richtet sich in erster Linie an Fachleute aus der chemischen Industrie, der öffentlichen Hand, der Hochschulen, Lehranstalten, Fachinstituten und Ausbildungszentren, der Spitäler sowie an die Ärzteschaft.

Weiterer Anziehungspunkt während der ILMAC ist das bereits traditionelle 'Basler Treffen für Chemische Technik', an dem ausgewiesene Fachleute zu den Themen 'Neue Werkstoffe - Neue Technologien' referieren.

Detaillierte Informationen erteilt das Sekretariat ILMAC, Postfach, $\mathrm{CH}-4021$ Basel, Tel. 061 6862020 , Telex $962685 \mathrm{smm}$ ch, Telefax 0616920617 . 


\section{Personalia}

\section{Geburtstage}

Hans-Rudolf Schmid, Dr. sc. techn., Riehen, Mitglied des SChV, feiert am 16.9.90 seinen 65. Geburtstag.

Max J. Ruther, Dr. rer. nat., Forch, Mitglied des SChV, feiert an 20.9.90 seinen 70. Geburtstag.

\section{Neue Mitglieder}

Ács Mária, Prof. Dr., Dept. Org. Chem. Technology, TU Budapest, Postfach 91, H-1521 Budapest.

Breitschuh Richard, Dipl. Chem., Sprensenbühl 10 8032 Zürich

Von dem Bussche-H. Christoph, Dipl. Chem., Herbstweg 72, 8050 Zürich.

\section{Wahlen}

Als ordentlicher Professor für informatikgestützte Chemie an der ETH Zürich wurde auf den 1. September 1990 gewählt: Wilfred F. van Gunsteren, Ph. D. geboren 1947, niederländischer Staatsbürger, zurzeit Professor für physikalische Chemie an der Universität Groningen NL.

Als ordentlicher Professor für allgemeine und anorganische Chemie an der ETH Zürich wurde auf den 1.Oktober 1990 gewählt: Reinhard Nesper, Dr. rer nat., geboren 1949, deutscher Staatsangehöriger, zurzeit wissenschaftlicher Mitarbeiter am Max-Planck-Institut für Festkörperforschung in Stuttgart.

PD Dr. Urs Séquin wurde zu einem ausserordentlichen Professor der Philosophisch-Naturwissenschaftlichen Fakultät der Universität Basel ernannt.

\section{Gründung einer Fachgruppe Rheologie}

Am 24. April 1990 fand in der ETH Zürich eine In formationstagung Rheologie statt, die von der Polymer-Gruppe der Schweiz (PGS) organisiert worden ist. Diese Veranstaltung sollte die an der Rheologie Interessierten zusammenführen und zeigen, auf welchen $\mathrm{Ge}$ bieten in der Schweiz rheologische Aktivitäten beste. hen.

Es wurden folgende Vorträge gehalten:

Prof. Dr. F. Escher

Institut für Lebensmittelwissenschaft

ETH Zürich

Rheologie in der Lebensmitteltechnologie

Prof. Dr. J. Meissner

Institut für Polymere, ETH Zürich

Messverfahren in der Rheologie von Polymer-Schmelzen

Dr. D. Hadjistamov

Ciba-Geigy AG, 4133 Schweizerhalle

Rheologie von formulierten dispersen Systemen

Dr. A. Gyr

Institut für Hydromechanik und Wasserbau

ETH-Zürich

Rheologie in der Turbulenzforschung von Wasser

Dr. Th. Raible

Geberit AG, 8640 Rapperswil

Rheologische Auslegung von Spritzgusswerkzeugen

Am Ende der Informationstagung wurde von den Anwesenden einstimmig der Beschluss gefasst, eine Fachgruppe Rheologie innerhalb der Polymer-Gruppe der Schweiz zu bilden. Gleichzeitig wurden folgende vier Mitglieder in die Leitung der Fachgruppe Rheologie gewählt:

Prof. Dr. F. Escher, Institut für Lebensmittelwissen schaft, ETH Zürich

Dr. D. Hadjistamov, Ciba-Geigy AG, 4133 Schweizerhalle

Prof. Dr. J. Meissner, Institut für Polymere, ETH Zürich

Dr. Th. Raible, Geberit AG, 8640 Rapperswil

Zum Leiter der Fachgruppe Rheologie wurde am 15. Mai 1990 bei der Sitzung der Gruppenleitung Prof Dr. J. Meissner gewählt, Herr Prof. Dr. F. Escher zu seinem Stellvertreter. Die Amtsperiode beträgt jeweils zwei Jahre, Wiederwahl ist zulässig.

Die Leitung der Fachgruppe sieht folgende zukünftige Aktivitäten für die Fachgruppe Rheologie:

1. Ausbildung in Rheologie auf Anfängerniveau,

2. Fortbildung für Fortgeschrittene,

3. Behandlung wissenschaftlicher und anwendungsbezogener Themen sowie spezieller Forschungsfragen,

4. Informationsveranstaltungen über bestimmte Themen mit (wenn möglich) Demonstrationen,

5. lokale Tagungen über spezielle Themen,

6. Organisation von Exkursionen zu ausgewählten Firmen.

Zur Frage der Mitglicdschaft sei festgehalten, dass jedes Mitglied der Polymer-Gruppe der Schweiz ohne zusätzliche Kosten Mitglied der Fachgruppe Rheologie werden kann. Dementsprechend setzt die Mitgliedschaft in der Fachgruppe Rheologie die Mitgliedschaft in der PGS voraus.

Zürich, 12. Juni 1990

Prof. Dr. J. Meissner

\section{Biotechnologie für Chemiker}

Die Abteilung für Chemie am Technikum Winterthur Ingenieurschule (TWI) bietet ein einjähriges Nachdiplomstudium an:

Dieses Studium dauert zwei Semester à 19 Wochen mit je 30 Wochenstunden. Es wird zum zweiten Mal vom 12.11.1990 bis 12.10.1991 durchgeführt.

Ausbildungsziel ist die theoretische und praktische Einführung in die Biotechnologie und deren Anwendung in den Gebieten Bioverfahrenstechnik, Analytik und Ökologie

Der Kurs richtet sich an Chemiker, die sich für praktische Anwendungen biotechnischer Verfahren interessieren, sowie an weitere Interessenten mit geeigneter Vorbildung und Praxis.

Dic Teilnehmerzahl ist auf 12 beschränkt

Interessenten erhalten weitere Informationen* bei:

Technikum Winterthur Ingenieurschule

Prof. Dr. G. Wolf

Postfach

CH-8401 Winterthur

Telephon (052) 8263 23/64, Kanzlei: (052) 826161

Telefax (052) 826383

*Vgl. dazu G.Wolf, CHIMIA 1988, 42, 342; 1990, 44 33 .

\section{Erfolgreiche Basler Chemie-Konzerne}

I.C. - Die drei grössten Schweizer Chemieunternehmen Ciba-Geigy, Hoffmann-La Roche und Sandoz konnten an ihren Bilanzpressekonferenzen für das Jahr 1989 wiederum sehr gute Erfolge präsentieren. Auf den internationalen Märkten haben die drei Konzerne Umsätze in Höhe von fast $43 \mathrm{Mrd}$. Franken erreicht. Das sind rund $6 \mathrm{Mrd}$. Franken oder $17 \%$ mehr als im Vorjahr. $40 \%$ dieses Wachstums sind allerdings währungsbedingt. Insgesamt beschäftigten die drei Basler Konzerne weltweit 193411 Mitarbeiterinnen und Mitarbeiter, davon 51976 oder $27 \%$ in der Schweiz.

Grosse Mittel, nämlich rund $4 \mathrm{Mrd}$. Franken, wurden weltweit in Sachanlagen investiert. Davon entfielen rund 1,37 Mrd. Franken oder 34\% auf Neueinrichtungen in Schweizer Chemiewerken, was ein volkswirtschaftlich beachtliches Volumen für das Baugewerbe und die Ausrüstungsindustrie darstellt.
Die Forschungs- und Entwicklungsaufwendungen beliefen sich 1989 auf insgesamt 4,6 Mrd. Franken oder $10,7 \%$ der Umsätze. Wie Ciba-Geigy in ihrem Geschäftsbericht schreibt, findet das Wachstum bei $F+E$ vor allem im Ausland statt, da in der Schweiz der beschränkt vorhandene Chemikernachwuchs diesem Gebiet Grenzen setzt

Beachtlich sind die Aufwendungen der Basler Stammhäuser mit rund $750 \mathrm{Mio}$. Franken für die Sicherheit und den Umweltschutz. Alle Unternehmensführungen betrachten den Schutz der Umwelt neben dem wirtschaftichen Erfolg als Teil der unternehmerischen Gesamtverantwortung. Produktesicherheit, Sicherheit bei der Prozessabwicklung und Umweltschutz in der Produktion sind heute feste Beiträge zum qualitativen Wachstum der Branche Chemie.

\section{ALUSUISSE-LONZA übernimmt Cyclo in USA}

Basel, 17. Juli 1990 - Der Bereich Organische Chemie der ALUSUISSE-LONZA-Gruppe hat durch Alusuisse of America lnc. die Cyclo Products Inc. und die Schwestergesellschaft Balenco Enterprises Inc. Lo Angeles, USA, übernommen. Diese Unternehmen sind auf die Herstellung von hochwertigen Zwischenprodukten und Bulk Generika spezialisiert und beschäftigen 60 Mitarbeiter bei einem Umsatz von annähernd USS 11 Mio. Die Aktivitäten der akquirierten Unternehmen passen sehr gut in die bestehende Produktepalette der LONZA AG. Mit der Akquisition kann der Bereich Organische Chemie der ALUSUISSE LONZA-Gruppe sein Sortiment auf dem Gebiete der Feinchemikalien erweitern und seine Position im amerikanischen Markt verbessern. Für den Vertrieb ihrer Produkte können beide Unternehmen das weltweite Distributionsnetz der ALUSUISSE-LONZA-Gruppe benutzen.

\section{ILMAC 90 - die Chemiefachmesse in der Chemiemetropole}

Basel, als europäische Chemie- und Pharmaziemetropole, ist der geeignete Durchführungsort für die ILMAC 90, 11. Internationale Chemiefachmesse für Laboratoriums- und Verfahrenstechnik, Messtechnik und Automatik, vom 23. bis 26. Oktober 1990 in den Hallen der Schweizer Mustermesse.

Die Vergangenheit hat gezeigt, dass dem Risikaabbau in den Produktionsprozessen der chemischen Industrie ein immer höherer Stellenwert beizumessen ist. Innovative und v. a. umweltfreundlichere Lösungen in der Verfahrens-, Mess-, Regel- und Automationstechnik drängen sich auf.

Im Zuge dieser dynamischen Entwicklung bietet die ILMAC eine Standortbestimmung. Als eine der international wichtigsten Fachmessen auf ihrem Gebiet fasst sie alle Bereiche der chemischen Produktion zusammen: Laborausstattung und -technik; Isotopen und Radiochemie; Verfahrenstechnik; Mess-, Regelund Automationstechnik; Biotechnologie; Labortierhaltung; Umwelt- und Gesundheitsschutz; Laboreinrichtungen für Arztpraxen sowie Dienstleistungen. Durch die Erschliessung eines neuen Sektors, Laboreinrichtungen für Arztpraxen, werden an der ILMAC in vermehrtem Masse die Ärzte angesprochen. Bei diesen besteht ein wachsendes Bedürfnis für entsprechende Einrichtungen in den Praxen.

Die im Dreijahresturnus in Zusammenarbeit mit dem Schweizerischen Chemiker-Verband veranstaltete ILMAC richtet sich in erster Linie an Fachleute aus der chemischen Industrie, der öffentlichen Hand, der Hochschulen, Lehranstalten, Fachinstituten und Ausbildungszentren, der Spitäler sowie an die Ärzteschaft.

Weiterer Anziehungspunkt während der ILMAC ist das bereits traditionelle 'Basler Treffen für Chemische Technik', an dem ausgewiesene Fachleute zu den Themen 'Neue Werkstoffe - Neue Technologien' referieren.

Detaillierte Informationen erteilt das Sekretariat ILMAC, Postfach, $\mathrm{CH}-4021$ Basel, Tel. 061 6862020 , Telex $962685 \mathrm{smm}$ ch, Telefax 0616920617 . 


\section{Personalia}

\section{Geburtstage}

Hans-Rudolf Schmid, Dr. sc. techn., Riehen, Mitglied des SChV, feiert am 16.9.90 seinen 65. Geburtstag.

Max J. Ruther, Dr. rer. nat., Forch, Mitglied des SChV, feiert an 20.9.90 seinen 70. Geburtstag.

\section{Neue Mitglieder}

Ács Mária, Prof. Dr., Dept. Org. Chem. Technology, TU Budapest, Postfach 91, H-1521 Budapest.

Breitschuh Richard, Dipl. Chem., Sprensenbühl 10 8032 Zürich

Von dem Bussche-H. Christoph, Dipl. Chem., Herbstweg 72, 8050 Zürich.

\section{Wahlen}

Als ordentlicher Professor für informatikgestützte Chemie an der ETH Zürich wurde auf den 1. September 1990 gewählt: Wilfred F. van Gunsteren, Ph. D. geboren 1947, niederländischer Staatsbürger, zurzeit Professor für physikalische Chemie an der Universität Groningen NL.

Als ordentlicher Professor für allgemeine und anorganische Chemie an der ETH Zürich wurde auf den 1.Oktober 1990 gewählt: Reinhard Nesper, Dr. rer nat., geboren 1949, deutscher Staatsangehöriger, zurzeit wissenschaftlicher Mitarbeiter am Max-Planck-Institut für Festkörperforschung in Stuttgart.

PD Dr. Urs Séquin wurde zu einem ausserordentlichen Professor der Philosophisch-Naturwissenschaftlichen Fakultät der Universität Basel ernannt.

\section{Gründung einer Fachgruppe Rheologie}

Am 24. April 1990 fand in der ETH Zürich eine In formationstagung Rheologie statt, die von der Polymer-Gruppe der Schweiz (PGS) organisiert worden ist. Diese Veranstaltung sollte die an der Rheologie Interessierten zusammenführen und zeigen, auf welchen $\mathrm{Ge}$ bieten in der Schweiz rheologische Aktivitäten beste. hen.

Es wurden folgende Vorträge gehalten:

Prof. Dr. F. Escher

Institut für Lebensmittelwissenschaft

ETH Zürich

Rheologie in der Lebensmitteltechnologie

Prof. Dr. J. Meissner

Institut für Polymere, ETH Zürich

Messverfahren in der Rheologie von Polymer-Schmelzen

Dr. D. Hadjistamov

Ciba-Geigy AG, 4133 Schweizerhalle

Rheologie von formulierten dispersen Systemen

Dr. A. Gyr

Institut für Hydromechanik und Wasserbau

ETH-Zürich

Rheologie in der Turbulenzforschung von Wasser

Dr. Th. Raible

Geberit AG, 8640 Rapperswil

Rheologische Auslegung von Spritzgusswerkzeugen

Am Ende der Informationstagung wurde von den Anwesenden einstimmig der Beschluss gefasst, eine Fachgruppe Rheologie innerhalb der Polymer-Gruppe der Schweiz zu bilden. Gleichzeitig wurden folgende vier Mitglieder in die Leitung der Fachgruppe Rheologie gewählt:

Prof. Dr. F. Escher, Institut für Lebensmittelwissen schaft, ETH Zürich

Dr. D. Hadjistamov, Ciba-Geigy AG, 4133 Schweizerhalle

Prof. Dr. J. Meissner, Institut für Polymere, ETH Zürich

Dr. Th. Raible, Geberit AG, 8640 Rapperswil

Zum Leiter der Fachgruppe Rheologie wurde am 15. Mai 1990 bei der Sitzung der Gruppenleitung Prof Dr. J. Meissner gewählt, Herr Prof. Dr. F. Escher zu seinem Stellvertreter. Die Amtsperiode beträgt jeweils zwei Jahre, Wiederwahl ist zulässig.

Die Leitung der Fachgruppe sieht folgende zukünftige Aktivitäten für die Fachgruppe Rheologie:

1. Ausbildung in Rheologie auf Anfängerniveau,

2. Fortbildung für Fortgeschrittene,

3. Behandlung wissenschaftlicher und anwendungsbezogener Themen sowie spezieller Forschungsfragen,

4. Informationsveranstaltungen über bestimmte Themen mit (wenn möglich) Demonstrationen,

5. lokale Tagungen über spezielle Themen,

6. Organisation von Exkursionen zu ausgewählten Firmen.

Zur Frage der Mitglicdschaft sei festgehalten, dass jedes Mitglied der Polymer-Gruppe der Schweiz ohne zusätzliche Kosten Mitglied der Fachgruppe Rheologie werden kann. Dementsprechend setzt die Mitgliedschaft in der Fachgruppe Rheologie die Mitgliedschaft in der PGS voraus.

Zürich, 12. Juni 1990

Prof. Dr. J. Meissner

\section{Biotechnologie für Chemiker}

Die Abteilung für Chemie am Technikum Winterthur Ingenieurschule (TWI) bietet ein einjähriges Nachdiplomstudium an:

Dieses Studium dauert zwei Semester à 19 Wochen mit je 30 Wochenstunden. Es wird zum zweiten Mal vom 12.11.1990 bis 12.10.1991 durchgeführt.

Ausbildungsziel ist die theoretische und praktische Einführung in die Biotechnologie und deren Anwendung in den Gebieten Bioverfahrenstechnik, Analytik und Ökologie

Der Kurs richtet sich an Chemiker, die sich für praktische Anwendungen biotechnischer Verfahren interessieren, sowie an weitere Interessenten mit geeigneter Vorbildung und Praxis.

Dic Teilnehmerzahl ist auf 12 beschränkt

Interessenten erhalten weitere Informationen* bei:

Technikum Winterthur Ingenieurschule

Prof. Dr. G. Wolf

Postfach

CH-8401 Winterthur

Telephon (052) 8263 23/64, Kanzlei: (052) 826161

Telefax (052) 826383

*Vgl. dazu G.Wolf, CHIMIA 1988, 42, 342; 1990, 44 33 .

\section{Erfolgreiche Basler Chemie-Konzerne}

I.C. - Die drei grössten Schweizer Chemieunternehmen Ciba-Geigy, Hoffmann-La Roche und Sandoz konnten an ihren Bilanzpressekonferenzen für das Jahr 1989 wiederum sehr gute Erfolge präsentieren. Auf den internationalen Märkten haben die drei Konzerne Umsätze in Höhe von fast $43 \mathrm{Mrd}$. Franken erreicht. Das sind rund $6 \mathrm{Mrd}$. Franken oder $17 \%$ mehr als im Vorjahr. $40 \%$ dieses Wachstums sind allerdings währungsbedingt. Insgesamt beschäftigten die drei Basler Konzerne weltweit 193411 Mitarbeiterinnen und Mitarbeiter, davon 51976 oder $27 \%$ in der Schweiz.

Grosse Mittel, nämlich rund $4 \mathrm{Mrd}$. Franken, wurden weltweit in Sachanlagen investiert. Davon entfielen rund 1,37 Mrd. Franken oder 34\% auf Neueinrichtungen in Schweizer Chemiewerken, was ein volkswirtschaftlich beachtliches Volumen für das Baugewerbe und die Ausrüstungsindustrie darstellt.
Die Forschungs- und Entwicklungsaufwendungen beliefen sich 1989 auf insgesamt 4,6 Mrd. Franken oder $10,7 \%$ der Umsätze. Wie Ciba-Geigy in ihrem Geschäftsbericht schreibt, findet das Wachstum bei $F+E$ vor allem im Ausland statt, da in der Schweiz der beschränkt vorhandene Chemikernachwuchs diesem Gebiet Grenzen setzt

Beachtlich sind die Aufwendungen der Basler Stammhäuser mit rund $750 \mathrm{Mio}$. Franken für die Sicherheit und den Umweltschutz. Alle Unternehmensführungen betrachten den Schutz der Umwelt neben dem wirtschaftichen Erfolg als Teil der unternehmerischen Gesamtverantwortung. Produktesicherheit, Sicherheit bei der Prozessabwicklung und Umweltschutz in der Produktion sind heute feste Beiträge zum qualitativen Wachstum der Branche Chemie.

\section{ALUSUISSE-LONZA übernimmt Cyclo in USA}

Basel, 17. Juli 1990 - Der Bereich Organische Chemie der ALUSUISSE-LONZA-Gruppe hat durch Alusuisse of America lnc. die Cyclo Products Inc. und die Schwestergesellschaft Balenco Enterprises Inc. Lo Angeles, USA, übernommen. Diese Unternehmen sind auf die Herstellung von hochwertigen Zwischenprodukten und Bulk Generika spezialisiert und beschäftigen 60 Mitarbeiter bei einem Umsatz von annähernd USS 11 Mio. Die Aktivitäten der akquirierten Unternehmen passen sehr gut in die bestehende Produktepalette der LONZA AG. Mit der Akquisition kann der Bereich Organische Chemie der ALUSUISSE LONZA-Gruppe sein Sortiment auf dem Gebiete der Feinchemikalien erweitern und seine Position im amerikanischen Markt verbessern. Für den Vertrieb ihrer Produkte können beide Unternehmen das weltweite Distributionsnetz der ALUSUISSE-LONZA-Gruppe benutzen.

\section{ILMAC 90 - die Chemiefachmesse in der Chemiemetropole}

Basel, als europäische Chemie- und Pharmaziemetropole, ist der geeignete Durchführungsort für die ILMAC 90, 11. Internationale Chemiefachmesse für Laboratoriums- und Verfahrenstechnik, Messtechnik und Automatik, vom 23. bis 26. Oktober 1990 in den Hallen der Schweizer Mustermesse.

Die Vergangenheit hat gezeigt, dass dem Risikaabbau in den Produktionsprozessen der chemischen Industrie ein immer höherer Stellenwert beizumessen ist. Innovative und v. a. umweltfreundlichere Lösungen in der Verfahrens-, Mess-, Regel- und Automationstechnik drängen sich auf.

Im Zuge dieser dynamischen Entwicklung bietet die ILMAC eine Standortbestimmung. Als eine der international wichtigsten Fachmessen auf ihrem Gebiet fasst sie alle Bereiche der chemischen Produktion zusammen: Laborausstattung und -technik; Isotopen und Radiochemie; Verfahrenstechnik; Mess-, Regelund Automationstechnik; Biotechnologie; Labortierhaltung; Umwelt- und Gesundheitsschutz; Laboreinrichtungen für Arztpraxen sowie Dienstleistungen. Durch die Erschliessung eines neuen Sektors, Laboreinrichtungen für Arztpraxen, werden an der ILMAC in vermehrtem Masse die Ärzte angesprochen. Bei diesen besteht ein wachsendes Bedürfnis für entsprechende Einrichtungen in den Praxen.

Die im Dreijahresturnus in Zusammenarbeit mit dem Schweizerischen Chemiker-Verband veranstaltete ILMAC richtet sich in erster Linie an Fachleute aus der chemischen Industrie, der öffentlichen Hand, der Hochschulen, Lehranstalten, Fachinstituten und Ausbildungszentren, der Spitäler sowie an die Ärzteschaft.

Weiterer Anziehungspunkt während der ILMAC ist das bereits traditionelle 'Basler Treffen für Chemische Technik', an dem ausgewiesene Fachleute zu den Themen 'Neue Werkstoffe - Neue Technologien' referieren.

Detaillierte Informationen erteilt das Sekretariat ILMAC, Postfach, $\mathrm{CH}-4021$ Basel, Tel. 061 6862020 , Telex $962685 \mathrm{smm}$ ch, Telefax 0616920617 . 


\section{Personalia}

\section{Geburtstage}

Hans-Rudolf Schmid, Dr. sc. techn., Riehen, Mitglied des SChV, feiert am 16.9.90 seinen 65. Geburtstag.

Max J. Ruther, Dr. rer. nat., Forch, Mitglied des SChV, feiert an 20.9.90 seinen 70. Geburtstag.

\section{Neue Mitglieder}

Ács Mária, Prof. Dr., Dept. Org. Chem. Technology, TU Budapest, Postfach 91, H-1521 Budapest.

Breitschuh Richard, Dipl. Chem., Sprensenbühl 10 8032 Zürich

Von dem Bussche-H. Christoph, Dipl. Chem., Herbstweg 72, 8050 Zürich.

\section{Wahlen}

Als ordentlicher Professor für informatikgestützte Chemie an der ETH Zürich wurde auf den 1. September 1990 gewählt: Wilfred F. van Gunsteren, Ph. D. geboren 1947, niederländischer Staatsbürger, zurzeit Professor für physikalische Chemie an der Universität Groningen NL.

Als ordentlicher Professor für allgemeine und anorganische Chemie an der ETH Zürich wurde auf den 1.Oktober 1990 gewählt: Reinhard Nesper, Dr. rer nat., geboren 1949, deutscher Staatsangehöriger, zurzeit wissenschaftlicher Mitarbeiter am Max-Planck-Institut für Festkörperforschung in Stuttgart.

PD Dr. Urs Séquin wurde zu einem ausserordentlichen Professor der Philosophisch-Naturwissenschaftlichen Fakultät der Universität Basel ernannt.

\section{Gründung einer Fachgruppe Rheologie}

Am 24. April 1990 fand in der ETH Zürich eine In formationstagung Rheologie statt, die von der Polymer-Gruppe der Schweiz (PGS) organisiert worden ist. Diese Veranstaltung sollte die an der Rheologie Interessierten zusammenführen und zeigen, auf welchen $\mathrm{Ge}$ bieten in der Schweiz rheologische Aktivitäten beste. hen.

Es wurden folgende Vorträge gehalten:

Prof. Dr. F. Escher

Institut für Lebensmittelwissenschaft

ETH Zürich

Rheologie in der Lebensmitteltechnologie

Prof. Dr. J. Meissner

Institut für Polymere, ETH Zürich

Messverfahren in der Rheologie von Polymer-Schmelzen

Dr. D. Hadjistamov

Ciba-Geigy AG, 4133 Schweizerhalle

Rheologie von formulierten dispersen Systemen

Dr. A. Gyr

Institut für Hydromechanik und Wasserbau

ETH-Zürich

Rheologie in der Turbulenzforschung von Wasser

Dr. Th. Raible

Geberit AG, 8640 Rapperswil

Rheologische Auslegung von Spritzgusswerkzeugen

Am Ende der Informationstagung wurde von den Anwesenden einstimmig der Beschluss gefasst, eine Fachgruppe Rheologie innerhalb der Polymer-Gruppe der Schweiz zu bilden. Gleichzeitig wurden folgende vier Mitglieder in die Leitung der Fachgruppe Rheologie gewählt:

Prof. Dr. F. Escher, Institut für Lebensmittelwissen schaft, ETH Zürich

Dr. D. Hadjistamov, Ciba-Geigy AG, 4133 Schweizerhalle

Prof. Dr. J. Meissner, Institut für Polymere, ETH Zürich

Dr. Th. Raible, Geberit AG, 8640 Rapperswil

Zum Leiter der Fachgruppe Rheologie wurde am 15. Mai 1990 bei der Sitzung der Gruppenleitung Prof Dr. J. Meissner gewählt, Herr Prof. Dr. F. Escher zu seinem Stellvertreter. Die Amtsperiode beträgt jeweils zwei Jahre, Wiederwahl ist zulässig.

Die Leitung der Fachgruppe sieht folgende zukünftige Aktivitäten für die Fachgruppe Rheologie:

1. Ausbildung in Rheologie auf Anfängerniveau,

2. Fortbildung für Fortgeschrittene,

3. Behandlung wissenschaftlicher und anwendungsbezogener Themen sowie spezieller Forschungsfragen,

4. Informationsveranstaltungen über bestimmte Themen mit (wenn möglich) Demonstrationen,

5. lokale Tagungen über spezielle Themen,

6. Organisation von Exkursionen zu ausgewählten Firmen.

Zur Frage der Mitglicdschaft sei festgehalten, dass jedes Mitglied der Polymer-Gruppe der Schweiz ohne zusätzliche Kosten Mitglied der Fachgruppe Rheologie werden kann. Dementsprechend setzt die Mitgliedschaft in der Fachgruppe Rheologie die Mitgliedschaft in der PGS voraus.

Zürich, 12. Juni 1990

Prof. Dr. J. Meissner

\section{Biotechnologie für Chemiker}

Die Abteilung für Chemie am Technikum Winterthur Ingenieurschule (TWI) bietet ein einjähriges Nachdiplomstudium an:

Dieses Studium dauert zwei Semester à 19 Wochen mit je 30 Wochenstunden. Es wird zum zweiten Mal vom 12.11.1990 bis 12.10.1991 durchgeführt.

Ausbildungsziel ist die theoretische und praktische Einführung in die Biotechnologie und deren Anwendung in den Gebieten Bioverfahrenstechnik, Analytik und Ökologie

Der Kurs richtet sich an Chemiker, die sich für praktische Anwendungen biotechnischer Verfahren interessieren, sowie an weitere Interessenten mit geeigneter Vorbildung und Praxis.

Dic Teilnehmerzahl ist auf 12 beschränkt

Interessenten erhalten weitere Informationen* bei:

Technikum Winterthur Ingenieurschule

Prof. Dr. G. Wolf

Postfach

CH-8401 Winterthur

Telephon (052) 8263 23/64, Kanzlei: (052) 826161

Telefax (052) 826383

*Vgl. dazu G.Wolf, CHIMIA 1988, 42, 342; 1990, 44 33 .

\section{Erfolgreiche Basler Chemie-Konzerne}

I.C. - Die drei grössten Schweizer Chemieunternehmen Ciba-Geigy, Hoffmann-La Roche und Sandoz konnten an ihren Bilanzpressekonferenzen für das Jahr 1989 wiederum sehr gute Erfolge präsentieren. Auf den internationalen Märkten haben die drei Konzerne Umsätze in Höhe von fast $43 \mathrm{Mrd}$. Franken erreicht. Das sind rund $6 \mathrm{Mrd}$. Franken oder $17 \%$ mehr als im Vorjahr. $40 \%$ dieses Wachstums sind allerdings währungsbedingt. Insgesamt beschäftigten die drei Basler Konzerne weltweit 193411 Mitarbeiterinnen und Mitarbeiter, davon 51976 oder $27 \%$ in der Schweiz.

Grosse Mittel, nämlich rund $4 \mathrm{Mrd}$. Franken, wurden weltweit in Sachanlagen investiert. Davon entfielen rund 1,37 Mrd. Franken oder 34\% auf Neueinrichtungen in Schweizer Chemiewerken, was ein volkswirtschaftlich beachtliches Volumen für das Baugewerbe und die Ausrüstungsindustrie darstellt.
Die Forschungs- und Entwicklungsaufwendungen beliefen sich 1989 auf insgesamt 4,6 Mrd. Franken oder $10,7 \%$ der Umsätze. Wie Ciba-Geigy in ihrem Geschäftsbericht schreibt, findet das Wachstum bei $F+E$ vor allem im Ausland statt, da in der Schweiz der beschränkt vorhandene Chemikernachwuchs diesem Gebiet Grenzen setzt

Beachtlich sind die Aufwendungen der Basler Stammhäuser mit rund $750 \mathrm{Mio}$. Franken für die Sicherheit und den Umweltschutz. Alle Unternehmensführungen betrachten den Schutz der Umwelt neben dem wirtschaftichen Erfolg als Teil der unternehmerischen Gesamtverantwortung. Produktesicherheit, Sicherheit bei der Prozessabwicklung und Umweltschutz in der Produktion sind heute feste Beiträge zum qualitativen Wachstum der Branche Chemie.

\section{ALUSUISSE-LONZA übernimmt Cyclo in USA}

Basel, 17. Juli 1990 - Der Bereich Organische Chemie der ALUSUISSE-LONZA-Gruppe hat durch Alusuisse of America lnc. die Cyclo Products Inc. und die Schwestergesellschaft Balenco Enterprises Inc. Lo Angeles, USA, übernommen. Diese Unternehmen sind auf die Herstellung von hochwertigen Zwischenprodukten und Bulk Generika spezialisiert und beschäftigen 60 Mitarbeiter bei einem Umsatz von annähernd USS 11 Mio. Die Aktivitäten der akquirierten Unternehmen passen sehr gut in die bestehende Produktepalette der LONZA AG. Mit der Akquisition kann der Bereich Organische Chemie der ALUSUISSE LONZA-Gruppe sein Sortiment auf dem Gebiete der Feinchemikalien erweitern und seine Position im amerikanischen Markt verbessern. Für den Vertrieb ihrer Produkte können beide Unternehmen das weltweite Distributionsnetz der ALUSUISSE-LONZA-Gruppe benutzen.

\section{ILMAC 90 - die Chemiefachmesse in der Chemiemetropole}

Basel, als europäische Chemie- und Pharmaziemetropole, ist der geeignete Durchführungsort für die ILMAC 90, 11. Internationale Chemiefachmesse für Laboratoriums- und Verfahrenstechnik, Messtechnik und Automatik, vom 23. bis 26. Oktober 1990 in den Hallen der Schweizer Mustermesse.

Die Vergangenheit hat gezeigt, dass dem Risikaabbau in den Produktionsprozessen der chemischen Industrie ein immer höherer Stellenwert beizumessen ist. Innovative und v. a. umweltfreundlichere Lösungen in der Verfahrens-, Mess-, Regel- und Automationstechnik drängen sich auf.

Im Zuge dieser dynamischen Entwicklung bietet die ILMAC eine Standortbestimmung. Als eine der international wichtigsten Fachmessen auf ihrem Gebiet fasst sie alle Bereiche der chemischen Produktion zusammen: Laborausstattung und -technik; Isotopen und Radiochemie; Verfahrenstechnik; Mess-, Regelund Automationstechnik; Biotechnologie; Labortierhaltung; Umwelt- und Gesundheitsschutz; Laboreinrichtungen für Arztpraxen sowie Dienstleistungen. Durch die Erschliessung eines neuen Sektors, Laboreinrichtungen für Arztpraxen, werden an der ILMAC in vermehrtem Masse die Ärzte angesprochen. Bei diesen besteht ein wachsendes Bedürfnis für entsprechende Einrichtungen in den Praxen.

Die im Dreijahresturnus in Zusammenarbeit mit dem Schweizerischen Chemiker-Verband veranstaltete ILMAC richtet sich in erster Linie an Fachleute aus der chemischen Industrie, der öffentlichen Hand, der Hochschulen, Lehranstalten, Fachinstituten und Ausbildungszentren, der Spitäler sowie an die Ärzteschaft.

Weiterer Anziehungspunkt während der ILMAC ist das bereits traditionelle 'Basler Treffen für Chemische Technik', an dem ausgewiesene Fachleute zu den Themen 'Neue Werkstoffe - Neue Technologien' referieren.

Detaillierte Informationen erteilt das Sekretariat ILMAC, Postfach, $\mathrm{CH}-4021$ Basel, Tel. 061 6862020 , Telex $962685 \mathrm{smm}$ ch, Telefax 0616920617 . 


\section{Personalia}

\section{Geburtstage}

Hans-Rudolf Schmid, Dr. sc. techn., Riehen, Mitglied des SChV, feiert am 16.9.90 seinen 65. Geburtstag.

Max J. Ruther, Dr. rer. nat., Forch, Mitglied des SChV, feiert an 20.9.90 seinen 70. Geburtstag.

\section{Neue Mitglieder}

Ács Mária, Prof. Dr., Dept. Org. Chem. Technology, TU Budapest, Postfach 91, H-1521 Budapest.

Breitschuh Richard, Dipl. Chem., Sprensenbühl 10 8032 Zürich

Von dem Bussche-H. Christoph, Dipl. Chem., Herbstweg 72, 8050 Zürich.

\section{Wahlen}

Als ordentlicher Professor für informatikgestützte Chemie an der ETH Zürich wurde auf den 1. September 1990 gewählt: Wilfred F. van Gunsteren, Ph. D. geboren 1947, niederländischer Staatsbürger, zurzeit Professor für physikalische Chemie an der Universität Groningen NL.

Als ordentlicher Professor für allgemeine und anorganische Chemie an der ETH Zürich wurde auf den 1.Oktober 1990 gewählt: Reinhard Nesper, Dr. rer nat., geboren 1949, deutscher Staatsangehöriger, zurzeit wissenschaftlicher Mitarbeiter am Max-Planck-Institut für Festkörperforschung in Stuttgart.

PD Dr. Urs Séquin wurde zu einem ausserordentlichen Professor der Philosophisch-Naturwissenschaftlichen Fakultät der Universität Basel ernannt.

\section{Gründung einer Fachgruppe Rheologie}

Am 24. April 1990 fand in der ETH Zürich eine In formationstagung Rheologie statt, die von der Polymer-Gruppe der Schweiz (PGS) organisiert worden ist. Diese Veranstaltung sollte die an der Rheologie Interessierten zusammenführen und zeigen, auf welchen $\mathrm{Ge}$ bieten in der Schweiz rheologische Aktivitäten beste. hen.

Es wurden folgende Vorträge gehalten:

Prof. Dr. F. Escher

Institut für Lebensmittelwissenschaft

ETH Zürich

Rheologie in der Lebensmitteltechnologie

Prof. Dr. J. Meissner

Institut für Polymere, ETH Zürich

Messverfahren in der Rheologie von Polymer-Schmelzen

Dr. D. Hadjistamov

Ciba-Geigy AG, 4133 Schweizerhalle

Rheologie von formulierten dispersen Systemen

Dr. A. Gyr

Institut für Hydromechanik und Wasserbau

ETH-Zürich

Rheologie in der Turbulenzforschung von Wasser

Dr. Th. Raible

Geberit AG, 8640 Rapperswil

Rheologische Auslegung von Spritzgusswerkzeugen

Am Ende der Informationstagung wurde von den Anwesenden einstimmig der Beschluss gefasst, eine Fachgruppe Rheologie innerhalb der Polymer-Gruppe der Schweiz zu bilden. Gleichzeitig wurden folgende vier Mitglieder in die Leitung der Fachgruppe Rheologie gewählt:

Prof. Dr. F. Escher, Institut für Lebensmittelwissen schaft, ETH Zürich

Dr. D. Hadjistamov, Ciba-Geigy AG, 4133 Schweizerhalle

Prof. Dr. J. Meissner, Institut für Polymere, ETH Zürich

Dr. Th. Raible, Geberit AG, 8640 Rapperswil

Zum Leiter der Fachgruppe Rheologie wurde am 15. Mai 1990 bei der Sitzung der Gruppenleitung Prof Dr. J. Meissner gewählt, Herr Prof. Dr. F. Escher zu seinem Stellvertreter. Die Amtsperiode beträgt jeweils zwei Jahre, Wiederwahl ist zulässig.

Die Leitung der Fachgruppe sieht folgende zukünftige Aktivitäten für die Fachgruppe Rheologie:

1. Ausbildung in Rheologie auf Anfängerniveau,

2. Fortbildung für Fortgeschrittene,

3. Behandlung wissenschaftlicher und anwendungsbezogener Themen sowie spezieller Forschungsfragen,

4. Informationsveranstaltungen über bestimmte Themen mit (wenn möglich) Demonstrationen,

5. lokale Tagungen über spezielle Themen,

6. Organisation von Exkursionen zu ausgewählten Firmen.

Zur Frage der Mitglicdschaft sei festgehalten, dass jedes Mitglied der Polymer-Gruppe der Schweiz ohne zusätzliche Kosten Mitglied der Fachgruppe Rheologie werden kann. Dementsprechend setzt die Mitgliedschaft in der Fachgruppe Rheologie die Mitgliedschaft in der PGS voraus.

Zürich, 12. Juni 1990

Prof. Dr. J. Meissner

\section{Biotechnologie für Chemiker}

Die Abteilung für Chemie am Technikum Winterthur Ingenieurschule (TWI) bietet ein einjähriges Nachdiplomstudium an:

Dieses Studium dauert zwei Semester à 19 Wochen mit je 30 Wochenstunden. Es wird zum zweiten Mal vom 12.11.1990 bis 12.10.1991 durchgeführt.

Ausbildungsziel ist die theoretische und praktische Einführung in die Biotechnologie und deren Anwendung in den Gebieten Bioverfahrenstechnik, Analytik und Ökologie

Der Kurs richtet sich an Chemiker, die sich für praktische Anwendungen biotechnischer Verfahren interessieren, sowie an weitere Interessenten mit geeigneter Vorbildung und Praxis.

Dic Teilnehmerzahl ist auf 12 beschränkt

Interessenten erhalten weitere Informationen* bei:

Technikum Winterthur Ingenieurschule

Prof. Dr. G. Wolf

Postfach

CH-8401 Winterthur

Telephon (052) 8263 23/64, Kanzlei: (052) 826161

Telefax (052) 826383

*Vgl. dazu G.Wolf, CHIMIA 1988, 42, 342; 1990, 44 33 .

\section{Erfolgreiche Basler Chemie-Konzerne}

I.C. - Die drei grössten Schweizer Chemieunternehmen Ciba-Geigy, Hoffmann-La Roche und Sandoz konnten an ihren Bilanzpressekonferenzen für das Jahr 1989 wiederum sehr gute Erfolge präsentieren. Auf den internationalen Märkten haben die drei Konzerne Umsätze in Höhe von fast $43 \mathrm{Mrd}$. Franken erreicht. Das sind rund $6 \mathrm{Mrd}$. Franken oder $17 \%$ mehr als im Vorjahr. $40 \%$ dieses Wachstums sind allerdings währungsbedingt. Insgesamt beschäftigten die drei Basler Konzerne weltweit 193411 Mitarbeiterinnen und Mitarbeiter, davon 51976 oder $27 \%$ in der Schweiz.

Grosse Mittel, nämlich rund $4 \mathrm{Mrd}$. Franken, wurden weltweit in Sachanlagen investiert. Davon entfielen rund 1,37 Mrd. Franken oder 34\% auf Neueinrichtungen in Schweizer Chemiewerken, was ein volkswirtschaftlich beachtliches Volumen für das Baugewerbe und die Ausrüstungsindustrie darstellt.
Die Forschungs- und Entwicklungsaufwendungen beliefen sich 1989 auf insgesamt 4,6 Mrd. Franken oder $10,7 \%$ der Umsätze. Wie Ciba-Geigy in ihrem Geschäftsbericht schreibt, findet das Wachstum bei $F+E$ vor allem im Ausland statt, da in der Schweiz der beschränkt vorhandene Chemikernachwuchs diesem Gebiet Grenzen setzt

Beachtlich sind die Aufwendungen der Basler Stammhäuser mit rund $750 \mathrm{Mio}$. Franken für die Sicherheit und den Umweltschutz. Alle Unternehmensführungen betrachten den Schutz der Umwelt neben dem wirtschaftichen Erfolg als Teil der unternehmerischen Gesamtverantwortung. Produktesicherheit, Sicherheit bei der Prozessabwicklung und Umweltschutz in der Produktion sind heute feste Beiträge zum qualitativen Wachstum der Branche Chemie.

\section{ALUSUISSE-LONZA übernimmt Cyclo in USA}

Basel, 17. Juli 1990 - Der Bereich Organische Chemie der ALUSUISSE-LONZA-Gruppe hat durch Alusuisse of America lnc. die Cyclo Products Inc. und die Schwestergesellschaft Balenco Enterprises Inc. Lo Angeles, USA, übernommen. Diese Unternehmen sind auf die Herstellung von hochwertigen Zwischenprodukten und Bulk Generika spezialisiert und beschäftigen 60 Mitarbeiter bei einem Umsatz von annähernd USS 11 Mio. Die Aktivitäten der akquirierten Unternehmen passen sehr gut in die bestehende Produktepalette der LONZA AG. Mit der Akquisition kann der Bereich Organische Chemie der ALUSUISSE LONZA-Gruppe sein Sortiment auf dem Gebiete der Feinchemikalien erweitern und seine Position im amerikanischen Markt verbessern. Für den Vertrieb ihrer Produkte können beide Unternehmen das weltweite Distributionsnetz der ALUSUISSE-LONZA-Gruppe benutzen.

\section{ILMAC 90 - die Chemiefachmesse in der Chemiemetropole}

Basel, als europäische Chemie- und Pharmaziemetropole, ist der geeignete Durchführungsort für die ILMAC 90, 11. Internationale Chemiefachmesse für Laboratoriums- und Verfahrenstechnik, Messtechnik und Automatik, vom 23. bis 26. Oktober 1990 in den Hallen der Schweizer Mustermesse.

Die Vergangenheit hat gezeigt, dass dem Risikaabbau in den Produktionsprozessen der chemischen Industrie ein immer höherer Stellenwert beizumessen ist. Innovative und v. a. umweltfreundlichere Lösungen in der Verfahrens-, Mess-, Regel- und Automationstechnik drängen sich auf.

Im Zuge dieser dynamischen Entwicklung bietet die ILMAC eine Standortbestimmung. Als eine der international wichtigsten Fachmessen auf ihrem Gebiet fasst sie alle Bereiche der chemischen Produktion zusammen: Laborausstattung und -technik; Isotopen und Radiochemie; Verfahrenstechnik; Mess-, Regelund Automationstechnik; Biotechnologie; Labortierhaltung; Umwelt- und Gesundheitsschutz; Laboreinrichtungen für Arztpraxen sowie Dienstleistungen. Durch die Erschliessung eines neuen Sektors, Laboreinrichtungen für Arztpraxen, werden an der ILMAC in vermehrtem Masse die Ärzte angesprochen. Bei diesen besteht ein wachsendes Bedürfnis für entsprechende Einrichtungen in den Praxen.

Die im Dreijahresturnus in Zusammenarbeit mit dem Schweizerischen Chemiker-Verband veranstaltete ILMAC richtet sich in erster Linie an Fachleute aus der chemischen Industrie, der öffentlichen Hand, der Hochschulen, Lehranstalten, Fachinstituten und Ausbildungszentren, der Spitäler sowie an die Ärzteschaft.

Weiterer Anziehungspunkt während der ILMAC ist das bereits traditionelle 'Basler Treffen für Chemische Technik', an dem ausgewiesene Fachleute zu den Themen 'Neue Werkstoffe - Neue Technologien' referieren.

Detaillierte Informationen erteilt das Sekretariat ILMAC, Postfach, $\mathrm{CH}-4021$ Basel, Tel. 061 6862020 , Telex $962685 \mathrm{smm}$ ch, Telefax 0616920617 . 


\section{Personalia}

\section{Geburtstage}

Hans-Rudolf Schmid, Dr. sc. techn., Riehen, Mitglied des SChV, feiert am 16.9.90 seinen 65. Geburtstag.

Max J. Ruther, Dr. rer. nat., Forch, Mitglied des SChV, feiert an 20.9.90 seinen 70. Geburtstag.

\section{Neue Mitglieder}

Ács Mária, Prof. Dr., Dept. Org. Chem. Technology, TU Budapest, Postfach 91, H-1521 Budapest.

Breitschuh Richard, Dipl. Chem., Sprensenbühl 10 8032 Zürich

Von dem Bussche-H. Christoph, Dipl. Chem., Herbstweg 72, 8050 Zürich.

\section{Wahlen}

Als ordentlicher Professor für informatikgestützte Chemie an der ETH Zürich wurde auf den 1. September 1990 gewählt: Wilfred F. van Gunsteren, Ph. D. geboren 1947, niederländischer Staatsbürger, zurzeit Professor für physikalische Chemie an der Universität Groningen NL.

Als ordentlicher Professor für allgemeine und anorganische Chemie an der ETH Zürich wurde auf den 1.Oktober 1990 gewählt: Reinhard Nesper, Dr. rer nat., geboren 1949, deutscher Staatsangehöriger, zurzeit wissenschaftlicher Mitarbeiter am Max-Planck-Institut für Festkörperforschung in Stuttgart.

PD Dr. Urs Séquin wurde zu einem ausserordentlichen Professor der Philosophisch-Naturwissenschaftlichen Fakultät der Universität Basel ernannt.

\section{Gründung einer Fachgruppe Rheologie}

Am 24. April 1990 fand in der ETH Zürich eine In formationstagung Rheologie statt, die von der Polymer-Gruppe der Schweiz (PGS) organisiert worden ist. Diese Veranstaltung sollte die an der Rheologie Interessierten zusammenführen und zeigen, auf welchen $\mathrm{Ge}$ bieten in der Schweiz rheologische Aktivitäten beste. hen.

Es wurden folgende Vorträge gehalten:

Prof. Dr. F. Escher

Institut für Lebensmittelwissenschaft

ETH Zürich

Rheologie in der Lebensmitteltechnologie

Prof. Dr. J. Meissner

Institut für Polymere, ETH Zürich

Messverfahren in der Rheologie von Polymer-Schmelzen

Dr. D. Hadjistamov

Ciba-Geigy AG, 4133 Schweizerhalle

Rheologie von formulierten dispersen Systemen

Dr. A. Gyr

Institut für Hydromechanik und Wasserbau

ETH-Zürich

Rheologie in der Turbulenzforschung von Wasser

Dr. Th. Raible

Geberit AG, 8640 Rapperswil

Rheologische Auslegung von Spritzgusswerkzeugen

Am Ende der Informationstagung wurde von den Anwesenden einstimmig der Beschluss gefasst, eine Fachgruppe Rheologie innerhalb der Polymer-Gruppe der Schweiz zu bilden. Gleichzeitig wurden folgende vier Mitglieder in die Leitung der Fachgruppe Rheologie gewählt:

Prof. Dr. F. Escher, Institut für Lebensmittelwissen schaft, ETH Zürich

Dr. D. Hadjistamov, Ciba-Geigy AG, 4133 Schweizerhalle

Prof. Dr. J. Meissner, Institut für Polymere, ETH Zürich

Dr. Th. Raible, Geberit AG, 8640 Rapperswil

Zum Leiter der Fachgruppe Rheologie wurde am 15. Mai 1990 bei der Sitzung der Gruppenleitung Prof Dr. J. Meissner gewählt, Herr Prof. Dr. F. Escher zu seinem Stellvertreter. Die Amtsperiode beträgt jeweils zwei Jahre, Wiederwahl ist zulässig.

Die Leitung der Fachgruppe sieht folgende zukünftige Aktivitäten für die Fachgruppe Rheologie:

1. Ausbildung in Rheologie auf Anfängerniveau,

2. Fortbildung für Fortgeschrittene,

3. Behandlung wissenschaftlicher und anwendungsbezogener Themen sowie spezieller Forschungsfragen,

4. Informationsveranstaltungen über bestimmte Themen mit (wenn möglich) Demonstrationen,

5. lokale Tagungen über spezielle Themen,

6. Organisation von Exkursionen zu ausgewählten Firmen.

Zur Frage der Mitglicdschaft sei festgehalten, dass jedes Mitglied der Polymer-Gruppe der Schweiz ohne zusätzliche Kosten Mitglied der Fachgruppe Rheologie werden kann. Dementsprechend setzt die Mitgliedschaft in der Fachgruppe Rheologie die Mitgliedschaft in der PGS voraus.

Zürich, 12. Juni 1990

Prof. Dr. J. Meissner

\section{Biotechnologie für Chemiker}

Die Abteilung für Chemie am Technikum Winterthur Ingenieurschule (TWI) bietet ein einjähriges Nachdiplomstudium an:

Dieses Studium dauert zwei Semester à 19 Wochen mit je 30 Wochenstunden. Es wird zum zweiten Mal vom 12.11.1990 bis 12.10.1991 durchgeführt.

Ausbildungsziel ist die theoretische und praktische Einführung in die Biotechnologie und deren Anwendung in den Gebieten Bioverfahrenstechnik, Analytik und Ökologie

Der Kurs richtet sich an Chemiker, die sich für praktische Anwendungen biotechnischer Verfahren interessieren, sowie an weitere Interessenten mit geeigneter Vorbildung und Praxis.

Dic Teilnehmerzahl ist auf 12 beschränkt

Interessenten erhalten weitere Informationen* bei:

Technikum Winterthur Ingenieurschule

Prof. Dr. G. Wolf

Postfach

CH-8401 Winterthur

Telephon (052) 8263 23/64, Kanzlei: (052) 826161

Telefax (052) 826383

*Vgl. dazu G.Wolf, CHIMIA 1988, 42, 342; 1990, 44 33 .

\section{Erfolgreiche Basler Chemie-Konzerne}

I.C. - Die drei grössten Schweizer Chemieunternehmen Ciba-Geigy, Hoffmann-La Roche und Sandoz konnten an ihren Bilanzpressekonferenzen für das Jahr 1989 wiederum sehr gute Erfolge präsentieren. Auf den internationalen Märkten haben die drei Konzerne Umsätze in Höhe von fast $43 \mathrm{Mrd}$. Franken erreicht. Das sind rund $6 \mathrm{Mrd}$. Franken oder $17 \%$ mehr als im Vorjahr. $40 \%$ dieses Wachstums sind allerdings währungsbedingt. Insgesamt beschäftigten die drei Basler Konzerne weltweit 193411 Mitarbeiterinnen und Mitarbeiter, davon 51976 oder $27 \%$ in der Schweiz.

Grosse Mittel, nämlich rund $4 \mathrm{Mrd}$. Franken, wurden weltweit in Sachanlagen investiert. Davon entfielen rund 1,37 Mrd. Franken oder 34\% auf Neueinrichtungen in Schweizer Chemiewerken, was ein volkswirtschaftlich beachtliches Volumen für das Baugewerbe und die Ausrüstungsindustrie darstellt.
Die Forschungs- und Entwicklungsaufwendungen beliefen sich 1989 auf insgesamt 4,6 Mrd. Franken oder $10,7 \%$ der Umsätze. Wie Ciba-Geigy in ihrem Geschäftsbericht schreibt, findet das Wachstum bei $F+E$ vor allem im Ausland statt, da in der Schweiz der beschränkt vorhandene Chemikernachwuchs diesem Gebiet Grenzen setzt

Beachtlich sind die Aufwendungen der Basler Stammhäuser mit rund $750 \mathrm{Mio}$. Franken für die Sicherheit und den Umweltschutz. Alle Unternehmensführungen betrachten den Schutz der Umwelt neben dem wirtschaftichen Erfolg als Teil der unternehmerischen Gesamtverantwortung. Produktesicherheit, Sicherheit bei der Prozessabwicklung und Umweltschutz in der Produktion sind heute feste Beiträge zum qualitativen Wachstum der Branche Chemie.

\section{ALUSUISSE-LONZA übernimmt Cyclo in USA}

Basel, 17. Juli 1990 - Der Bereich Organische Chemie der ALUSUISSE-LONZA-Gruppe hat durch Alusuisse of America lnc. die Cyclo Products Inc. und die Schwestergesellschaft Balenco Enterprises Inc. Lo Angeles, USA, übernommen. Diese Unternehmen sind auf die Herstellung von hochwertigen Zwischenprodukten und Bulk Generika spezialisiert und beschäftigen 60 Mitarbeiter bei einem Umsatz von annähernd USS 11 Mio. Die Aktivitäten der akquirierten Unternehmen passen sehr gut in die bestehende Produktepalette der LONZA AG. Mit der Akquisition kann der Bereich Organische Chemie der ALUSUISSE LONZA-Gruppe sein Sortiment auf dem Gebiete der Feinchemikalien erweitern und seine Position im amerikanischen Markt verbessern. Für den Vertrieb ihrer Produkte können beide Unternehmen das weltweite Distributionsnetz der ALUSUISSE-LONZA-Gruppe benutzen.

\section{ILMAC 90 - die Chemiefachmesse in der Chemiemetropole}

Basel, als europäische Chemie- und Pharmaziemetropole, ist der geeignete Durchführungsort für die ILMAC 90, 11. Internationale Chemiefachmesse für Laboratoriums- und Verfahrenstechnik, Messtechnik und Automatik, vom 23. bis 26. Oktober 1990 in den Hallen der Schweizer Mustermesse.

Die Vergangenheit hat gezeigt, dass dem Risikaabbau in den Produktionsprozessen der chemischen Industrie ein immer höherer Stellenwert beizumessen ist. Innovative und v. a. umweltfreundlichere Lösungen in der Verfahrens-, Mess-, Regel- und Automationstechnik drängen sich auf.

Im Zuge dieser dynamischen Entwicklung bietet die ILMAC eine Standortbestimmung. Als eine der international wichtigsten Fachmessen auf ihrem Gebiet fasst sie alle Bereiche der chemischen Produktion zusammen: Laborausstattung und -technik; Isotopen und Radiochemie; Verfahrenstechnik; Mess-, Regelund Automationstechnik; Biotechnologie; Labortierhaltung; Umwelt- und Gesundheitsschutz; Laboreinrichtungen für Arztpraxen sowie Dienstleistungen. Durch die Erschliessung eines neuen Sektors, Laboreinrichtungen für Arztpraxen, werden an der ILMAC in vermehrtem Masse die Ärzte angesprochen. Bei diesen besteht ein wachsendes Bedürfnis für entsprechende Einrichtungen in den Praxen.

Die im Dreijahresturnus in Zusammenarbeit mit dem Schweizerischen Chemiker-Verband veranstaltete ILMAC richtet sich in erster Linie an Fachleute aus der chemischen Industrie, der öffentlichen Hand, der Hochschulen, Lehranstalten, Fachinstituten und Ausbildungszentren, der Spitäler sowie an die Ärzteschaft.

Weiterer Anziehungspunkt während der ILMAC ist das bereits traditionelle 'Basler Treffen für Chemische Technik', an dem ausgewiesene Fachleute zu den Themen 'Neue Werkstoffe - Neue Technologien' referieren.

Detaillierte Informationen erteilt das Sekretariat ILMAC, Postfach, $\mathrm{CH}-4021$ Basel, Tel. 061 6862020 , Telex $962685 \mathrm{smm}$ ch, Telefax 0616920617 . 


\section{Aufträge für Ems-Inventa AG aus Indien}

Das Schweizer Ingenieurunternehmen Ems-Invent AG - eine Gesellschaft der in den Bereichen Polymer Werkstoffe, Feinchemikalien und Engineering tätigen Ems-Gruppe - hat insgesamt 4 Aufträge von den indischen Firmen Haryana Petrochemicals Ltd. und Parasrampuria Synthetics Ltd. erhalten.

Es handelt sich um verschiedene Anlagen zur Herstellung von Polyestergranulat und Polyestergarnen.

Ems-Inventa AG stellt die Technologie, liefert die verfahrensmässig bedingten Maschinen und Einrichtungen, erbringt die Planungs- und Ingenieurarbeiten und stellt das Fachpersonal für die Überwachung der Montage und Inbetriebnahme.

Der gesamte Auftragswert stellt sich auf rund 25 Mio. SFr. Die Anlagen werden in den Staaten Haryana und Rajasthan errichtet. Die Produktionsaufnahme ist innerhalb von 2 Jahren geplant.

\section{Am Montag wurde 'Blau' gemacht:} Indigo, der König der Farbstoffe

I.C. - 'Indigo' - von diesem Wort ging über Jahrhunderte eine ungewöhnliche Faszination aus. Indigo, das war der teure Farbstoff aus fernen Ländern, der den Menschen die Farbe brachte, die sie überall sahen, aber nicht herstellen konnten. Inzwischen jedoch, vor allem dank der Jeans-Welle, ist Indigo ein Massenprodukt, dessen Synthese am 6. Mai dieses Jahres 100 Jahres al wurde.

Schon von jeher hat der Mensch das Bestreben gehabt, scine Unwelt durch Farben zu gestalten. Und während es ihm bereits vor mehr als 10000 Jahren gelang, rote, gelbe, braune und schwarze Farbtöne herzustellen, fehlte ihm lange Zeit das Blau.

In der Natur gibt es diesen Farbstoff - doch musst dessen Synthese erst gefunden werden. In zwei Pflanzenfamilien befindet sich nämlich eine farblose Substanz .. ein Vorprodukt, das heute Indoxyl genann wird. Vom farblosen Vorprodukt zur Farbe gelangte man durch zwei Schritte: zunächst wurde das Pflanzenmaterial zerstossen und dann unter Zusatz von Urin vergoren. Da dieser Prozess in Kübeln stattfand, nannte man die Brühe $K$ üpe. Tränkte man nun gesponnenes oder gewebtes Fasermaterial mit dieser immer noch farblosen Brühe und hängte es dann in die Sonne geschah - durch Oxidation, wie wir heute wissen - das Wunder: ein blauer Farbton entstand.

Bei den indoxylhaltigen Pflanzen handelt es sich zum einen um die zur Familie der Schmetterlingsblütler zählende Indigofera tinctoria, zum anderen um die mi den Kohlgewächsen verwandte Isatis tinctoria au
Deutsch: Färberwaid. Färberwaid wurde in Deutschand im Mittelalter auf grossen Flächen angebaut. Er enthält aber viel weniger farbgebende Substanz als da in den Subtropen wachsende Indigofera tinctoria, das jahrhundertelang in Indien, Indonesien und später auch in den USA landwirtschaftlich angebaut wurde.

\section{Indiga - begehrt und teuer}

Seinen Ursprung hatte das Indigo-Färben im 3. Jahr ' tausend vor unserer Zeitrechnung in - wie der Name erahnen lässt - Indien. Von hier aus trat es ganz langsam seinen Vormarsch nach Westen an und kam erst über den Landweg, dann mit der Entdeckung des Seeweges nach Indien durch Vasco da Gama 1498 nach Europa. Indigo wurde ein begehrtes Handelsproduk und verdrängte immer mehr den heimischen Färberwaid, dessen Blau auch nicht so schön und klar war wie das seines indischen Konkurrenten. Und weil das Blau so selten bzw. so mühsam zu beschaffen war, war es sehr teuer und damit die Farbe der herrschenden Klasse.

Von der Blaufärberei zur industriellen Produktion

Die Färber der damaligen Zeit hatten ein schwere Los. Nach zwölfstündigem, arbeitsintensivem Ansetzen der Küpe und dem Behandeln des Tuches in der Küpe folgte das ebenso lange Aushängen an der Luft Und weil am arbeitsfreien Sonntag das Färbegut länge als sonst - nämlich den ganzen Tag über - in der Küpe blieb, wurde am Montag dann 'Blau gemacht'. Die Gesellen überliessen der Luft den wesentlichen Teil de Arbeit, starteten relativ ruhig in die neue Woche und überlieferten uns so den 'blauen Montag'.

Um die Qualität des Indigo in den Griff und gleichmässigere Färbungen zu bekommen, befasste sich bald die wissenschaftliche Chemie mit dem Indigo. Und da der damals einfachste Weg, eine Substanz zu reinigen deren Verdampfen und anschliessendes Kondensieren war, unterwarf der Apotheker Unverdorben im Jah 1826 den Indigo dieser Prozedur. Der Indigo zersetzte sich dabei zu einer Verbindung, die der Apotheker Anilin nannte, angelehnt an das portugiesische Wort fü Blau. Der Weg in die richtige Richtung für einen ganzen Industriezweig war gewiesen.

\section{Das erste Patent}

Zahlreiche Forscher machten sich an die Arbeit. Es gelang, Anilin aus dem Teer zu gewinnen, der bei der Verkokung yon Steinkohle entstunden war. Erfolge blieben nicht aus. Viele Farbstoffe liessen sich synthetisieren, doch Blau war nicht dabei. Dem Hochschullehrer Adolf von Baeyer gelang es als erstem, das chemische Grundgerüst des Indigo zu entschlüsseln. Nachdem die Molekülstruktur erst einmal bekannt war, liess der nächste Schritt nicht lange auf sich warten. Im März 1880 wurde ihm für den von ihm gefundenen allerdings nicht praktikablen - Syntheseweg ein Paten erteilt. Man hatte 'Blau geleckt'. Weitere neue Synthesewege wurden entdeckt, unter anderem auch von dem Zürcher Professor Karl Heumann Am 6. Mai 1890 liess er sich seinen Weg patentieren, den die chemische Industrie nach weiteren sieben Jahren intensiver Forschung und Entwicklung technisch umsetzen konnte. Der erste künstliche Indigo war auf dem Markt.

Indigo wurde ein Markterfolg. Wichtige Exportlïnder waren China und Amerika, wo der Goldrausch i Kalifornien dem Indigo Aufschwung verlieh. Der ius Bayern nach San Francisco ausgewunderte Levi Strauss fertigte aus mit Indigo gefärbtem Drillich, den er aus dem Textilzentrum Nîmes bezog, die benötigle Arbeitskleidung. Von 'de Nimes' wurde dann in kurzer Zeit der Begriff 'Denim' und aus dem über Genua nach Amerika verschifften Baumwollstoff 'Bleu de Gènes' wurde schncll 'Blue Jeans'.

Jeans das Comeback eines Farbstoffes

Doch mit der Blütezeit des Indigo war es bald vorbci andere blaue Farbstoffe mit besseren Echtheiten wurden erfunden. Und so schwebte lange Zeit das Damoklesschwert der Produktionseinstcllung an cinem hauchdünnen Faden über den Indigofabriken. Abe dann kam in den 60er Jabren das grosse Comeback de Jeans. Die Kleidung, mit dem lichtechten Farbstof Indigo gefärbt, wandelte sich zur Uniform der Nonkonformisten. Die Jeans wurden zum Ausdruck eine veränderten Einstellung, kritisch und unkonventionell, und gehören inzwischen zur legeren Freizcitmode. Dank diesem Comeback wird Indigo heute immer noch grosstechnisch hergestellt.

\section{Schweizer Chemie auf ausreichende Stromversorgung angewiesen}

I.C. - Dank Verfahrensverbesserungen und Sparanstrengungen verbraucht die chemische lndustric in der Schweiz heute gesamthaft nicht mehr Energie als vor zehn Jahren, obwohl im gleichen Zeitraum die Produktion um rund $50 \%$ zunahm. Der Verbrauch an clektrischem Strom nahm allerdings um $27.7 \%$ zu, dies nich zuletzt als Folge der Anstrengungen zum Schutz der Unwelt wie beispielsweise der Betrieb yon Abwasserreinigungsanlagen oder von Elektrofiltern. Weiteres Stromsparen ist nach den jahrelangen Energiesparaktionen in der chemischen Industric kaum mehr möglich. Sic braucht Strom, und zwar aus allen Quellen.

Die chemische Industrie der Schweiz lehnt deshalb die beiden Anti-Atominitiativen (Moratorium und Ausstiegsinitiative) ab, die am 23. September 1990 zur Abstimmung gelangen. Die Schaffung eines Energieartikels in der Bundesverfassung erachtet sic als unnötig ordnungspolitisch verfehlt und wirtschaftlich gefährlich.

\section{Markt: Apparate, Chemikalien und Dienstleistungen}

Mettler-Toledo (Schweiz) AG
neue Adresse ab 1.8.1990:
Mettler-Toledo (Schweiz) AG
Waagen, Wägesysteme und analy-
tische Instrumente, Grabenstrasse 8,
CH-8606 Nänikon-Uster

Produktepalette und Neuheiten von Hamilton Medical

Hamilton Medical entwickelt und vertreibt umfassend die folgenden Geräte: Veolar, das perfekte Hochleistungs-Beatmungsgerät mit allen Extras, die konventionelle und zukünftige Beatmungstechniken erfordern und sicher ausführen. Eine optimale Auswahl an Beatmungsarten für die unterschiedlichsten Anforderungen. - Leonardo, der Patienten Data Manager zur Diagnostik für die Intensivpflegestation IPS. Leonardo er- laubt die Optimierung der Beatmung. Amadeus, der zuverlässige Routine-R spirator für die Intensivstation. Beinhaltet alle konventionellen Beatmungsar ten, Alarme und Monitoring, die für eine sichere, dem Patienten optimal angepasste Beatmung erforderlich sind. Max, der leistungsstarke portable Respirator für Notfall, Intensivmedizin un Transport. Optimale Ventilation selbs unter ungünstigsten Gegebenheiten Einzigartige Kombination von bewäh ter Elcktronik und Präzisionsmechanik. - Neu hat Hamilton eine Dilutoren-Dispensoren-Generation auf den Markt gebracht. Diese Geräteserie wird sowoh als Einzelgeräte mit Kontroller wie auch als OEM-Einbaumodelle gebaut. - De Hamilton Reader HR-7000 gehört zu den präzisesten Mikrotiterplatten-Photometern. Er eignet sich für alle Arte von optischen Dichtemessungen in Mikrotiterplatten. Zusammen mit den welt weit verkauften ML-AT und ML-2200 steilt er ein sehr ökonomisches System ur Blutbanken, Virologielabors und klinische Labors dar. Für die Entwicklung und Produktion von pH-Elektroden setzt Hamilton, neben einem aus Spitzenkräften der Branche zusammengesetzten Forschungs- und Entwicklungsteam, drei Jahrzehnte Erfahrung in Präzisions-Glasbearbeitung ein. Das Ergebnis: dauerhafte und präzise Sensoren zu erstaunlichen Preisen - Der Microlab AT/plus ist Hamiltons neuste Entwick lung. Er ist in der Lage, sehr effizient drei Abbott-Platten zu verarbeiten. RIA-Benutzer profitieren jetzt von einem aussergewöhnlich schnellen Gerät, dessen Potential vor allem darin besteht dass die Anwendungsmöglichkeiten stark erweitert worden sind

Hamilton Medical AG, Via Nova, CH-7403 Rhäzüns, Tel. (081) 372627, Telex 851321 hmedch, Telefax (081) 372689 . Leserdienst 51
Neue Umformer für den Anschluss von Strömungs-/ Durchflussmesswertaufnehmern

Dic Umformer sind für den Anschluss Don Höntzsch-Flügelrad-Messwertaufnehmern (FA) zur Messung der Strömungsgeschwindigkeit oder des Volumenstroms von Luft/Gasen und WasseFlüssigkeiten bestimnt. Sie wandeln die zur Strömungsgeschwindigkeit proportionale Signalírequenz eines Gebers (FA) in einen eingeprägten Strom ode eine eingeprägte Spannung um. Als Anaogsignale stchen nach Wahl 4-20 mA $0-4 \mathrm{~V}, 0-10 \mathrm{~V}$ zur Verfügung. Das Bezugspotential für den Spannungsausgang ist getrennt vom Bezugspotentia für die Versorgung. Die Versorgung kann nach Wahl durch 220 VAC, 24 VAC, 5 VDC, 12 bis 24 VDC erfolgen. In allen Fällen der Fremdspeisung sind die Versorgungsleitungen galvanisch ge- 


\section{Aufträge für Ems-Inventa AG aus Indien}

Das Schweizer Ingenieurunternehmen Ems-Invent AG - eine Gesellschaft der in den Bereichen Polymer Werkstoffe, Feinchemikalien und Engineering tätigen Ems-Gruppe - hat insgesamt 4 Aufträge von den indischen Firmen Haryana Petrochemicals Ltd. und Parasrampuria Synthetics Ltd. erhalten.

Es handelt sich um verschiedene Anlagen zur Herstellung von Polyestergranulat und Polyestergarnen.

Ems-Inventa AG stellt die Technologie, liefert die verfahrensmässig bedingten Maschinen und Einrichtungen, erbringt die Planungs- und Ingenieurarbeiten und stellt das Fachpersonal für die Überwachung der Montage und Inbetriebnahme.

Der gesamte Auftragswert stellt sich auf rund 25 Mio. SFr. Die Anlagen werden in den Staaten Haryana und Rajasthan errichtet. Die Produktionsaufnahme ist innerhalb von 2 Jahren geplant.

\section{Am Montag wurde 'Blau' gemacht:} Indigo, der König der Farbstoffe

I.C. - 'Indigo' - von diesem Wort ging über Jahrhunderte eine ungewöhnliche Faszination aus. Indigo, das war der teure Farbstoff aus fernen Ländern, der den Menschen die Farbe brachte, die sie überall sahen, aber nicht herstellen konnten. Inzwischen jedoch, vor allem dank der Jeans-Welle, ist Indigo ein Massenprodukt, dessen Synthese am 6. Mai dieses Jahres 100 Jahres al wurde.

Schon von jeher hat der Mensch das Bestreben gehabt, scine Unwelt durch Farben zu gestalten. Und während es ihm bereits vor mehr als 10000 Jahren gelang, rote, gelbe, braune und schwarze Farbtöne herzustellen, fehlte ihm lange Zeit das Blau.

In der Natur gibt es diesen Farbstoff - doch musst dessen Synthese erst gefunden werden. In zwei Pflanzenfamilien befindet sich nämlich eine farblose Substanz .. ein Vorprodukt, das heute Indoxyl genann wird. Vom farblosen Vorprodukt zur Farbe gelangte man durch zwei Schritte: zunächst wurde das Pflanzenmaterial zerstossen und dann unter Zusatz von Urin vergoren. Da dieser Prozess in Kübeln stattfand, nannte man die Brühe $K$ üpe. Tränkte man nun gesponnenes oder gewebtes Fasermaterial mit dieser immer noch farblosen Brühe und hängte es dann in die Sonne geschah - durch Oxidation, wie wir heute wissen - das Wunder: ein blauer Farbton entstand.

Bei den indoxylhaltigen Pflanzen handelt es sich zum einen um die zur Familie der Schmetterlingsblütler zählende Indigofera tinctoria, zum anderen um die mi den Kohlgewächsen verwandte Isatis tinctoria au
Deutsch: Färberwaid. Färberwaid wurde in Deutschand im Mittelalter auf grossen Flächen angebaut. Er enthält aber viel weniger farbgebende Substanz als da in den Subtropen wachsende Indigofera tinctoria, das jahrhundertelang in Indien, Indonesien und später auch in den USA landwirtschaftlich angebaut wurde.

\section{Indiga - begehrt und teuer}

Seinen Ursprung hatte das Indigo-Färben im 3. Jahr ' tausend vor unserer Zeitrechnung in - wie der Name erahnen lässt - Indien. Von hier aus trat es ganz langsam seinen Vormarsch nach Westen an und kam erst über den Landweg, dann mit der Entdeckung des Seeweges nach Indien durch Vasco da Gama 1498 nach Europa. Indigo wurde ein begehrtes Handelsproduk und verdrängte immer mehr den heimischen Färberwaid, dessen Blau auch nicht so schön und klar war wie das seines indischen Konkurrenten. Und weil das Blau so selten bzw. so mühsam zu beschaffen war, war es sehr teuer und damit die Farbe der herrschenden Klasse.

Von der Blaufärberei zur industriellen Produktion

Die Färber der damaligen Zeit hatten ein schwere Los. Nach zwölfstündigem, arbeitsintensivem Ansetzen der Küpe und dem Behandeln des Tuches in der Küpe folgte das ebenso lange Aushängen an der Luft Und weil am arbeitsfreien Sonntag das Färbegut länge als sonst - nämlich den ganzen Tag über - in der Küpe blieb, wurde am Montag dann 'Blau gemacht'. Die Gesellen überliessen der Luft den wesentlichen Teil de Arbeit, starteten relativ ruhig in die neue Woche und überlieferten uns so den 'blauen Montag'.

Um die Qualität des Indigo in den Griff und gleichmässigere Färbungen zu bekommen, befasste sich bald die wissenschaftliche Chemie mit dem Indigo. Und da der damals einfachste Weg, eine Substanz zu reinigen deren Verdampfen und anschliessendes Kondensieren war, unterwarf der Apotheker Unverdorben im Jah 1826 den Indigo dieser Prozedur. Der Indigo zersetzte sich dabei zu einer Verbindung, die der Apotheker Anilin nannte, angelehnt an das portugiesische Wort fü Blau. Der Weg in die richtige Richtung für einen ganzen Industriezweig war gewiesen.

\section{Das erste Patent}

Zahlreiche Forscher machten sich an die Arbeit. Es gelang, Anilin aus dem Teer zu gewinnen, der bei der Verkokung yon Steinkohle entstunden war. Erfolge blieben nicht aus. Viele Farbstoffe liessen sich synthetisieren, doch Blau war nicht dabei. Dem Hochschullehrer Adolf von Baeyer gelang es als erstem, das chemische Grundgerüst des Indigo zu entschlüsseln. Nachdem die Molekülstruktur erst einmal bekannt war, liess der nächste Schritt nicht lange auf sich warten. Im März 1880 wurde ihm für den von ihm gefundenen allerdings nicht praktikablen - Syntheseweg ein Paten erteilt. Man hatte 'Blau geleckt'. Weitere neue Synthesewege wurden entdeckt, unter anderem auch von dem Zürcher Professor Karl Heumann Am 6. Mai 1890 liess er sich seinen Weg patentieren, den die chemische Industrie nach weiteren sieben Jahren intensiver Forschung und Entwicklung technisch umsetzen konnte. Der erste künstliche Indigo war auf dem Markt.

Indigo wurde ein Markterfolg. Wichtige Exportlïnder waren China und Amerika, wo der Goldrausch i Kalifornien dem Indigo Aufschwung verlieh. Der ius Bayern nach San Francisco ausgewunderte Levi Strauss fertigte aus mit Indigo gefärbtem Drillich, den er aus dem Textilzentrum Nîmes bezog, die benötigle Arbeitskleidung. Von 'de Nimes' wurde dann in kurzer Zeit der Begriff 'Denim' und aus dem über Genua nach Amerika verschifften Baumwollstoff 'Bleu de Gènes' wurde schncll 'Blue Jeans'.

Jeans das Comeback eines Farbstoffes

Doch mit der Blütezeit des Indigo war es bald vorbci andere blaue Farbstoffe mit besseren Echtheiten wurden erfunden. Und so schwebte lange Zeit das Damoklesschwert der Produktionseinstcllung an cinem hauchdünnen Faden über den Indigofabriken. Abe dann kam in den 60er Jabren das grosse Comeback de Jeans. Die Kleidung, mit dem lichtechten Farbstof Indigo gefärbt, wandelte sich zur Uniform der Nonkonformisten. Die Jeans wurden zum Ausdruck eine veränderten Einstellung, kritisch und unkonventionell, und gehören inzwischen zur legeren Freizcitmode. Dank diesem Comeback wird Indigo heute immer noch grosstechnisch hergestellt.

\section{Schweizer Chemie auf ausreichende Stromversorgung angewiesen}

I.C. - Dank Verfahrensverbesserungen und Sparanstrengungen verbraucht die chemische lndustric in der Schweiz heute gesamthaft nicht mehr Energie als vor zehn Jahren, obwohl im gleichen Zeitraum die Produktion um rund $50 \%$ zunahm. Der Verbrauch an clektrischem Strom nahm allerdings um $27.7 \%$ zu, dies nich zuletzt als Folge der Anstrengungen zum Schutz der Unwelt wie beispielsweise der Betrieb yon Abwasserreinigungsanlagen oder von Elektrofiltern. Weiteres Stromsparen ist nach den jahrelangen Energiesparaktionen in der chemischen Industric kaum mehr möglich. Sic braucht Strom, und zwar aus allen Quellen.

Die chemische Industrie der Schweiz lehnt deshalb die beiden Anti-Atominitiativen (Moratorium und Ausstiegsinitiative) ab, die am 23. September 1990 zur Abstimmung gelangen. Die Schaffung eines Energieartikels in der Bundesverfassung erachtet sic als unnötig ordnungspolitisch verfehlt und wirtschaftlich gefährlich.

\section{Markt: Apparate, Chemikalien und Dienstleistungen}

Mettler-Toledo (Schweiz) AG
neue Adresse ab 1.8.1990:
Mettler-Toledo (Schweiz) AG
Waagen, Wägesysteme und analy-
tische Instrumente, Grabenstrasse 8,
CH-8606 Nänikon-Uster

Produktepalette und Neuheiten von Hamilton Medical

Hamilton Medical entwickelt und vertreibt umfassend die folgenden Geräte: Veolar, das perfekte Hochleistungs-Beatmungsgerät mit allen Extras, die konventionelle und zukünftige Beatmungstechniken erfordern und sicher ausführen. Eine optimale Auswahl an Beatmungsarten für die unterschiedlichsten Anforderungen. - Leonardo, der Patienten Data Manager zur Diagnostik für die Intensivpflegestation IPS. Leonardo er- laubt die Optimierung der Beatmung. Amadeus, der zuverlässige Routine-R spirator für die Intensivstation. Beinhaltet alle konventionellen Beatmungsar ten, Alarme und Monitoring, die für eine sichere, dem Patienten optimal angepasste Beatmung erforderlich sind. Max, der leistungsstarke portable Respirator für Notfall, Intensivmedizin un Transport. Optimale Ventilation selbs unter ungünstigsten Gegebenheiten Einzigartige Kombination von bewäh ter Elcktronik und Präzisionsmechanik. - Neu hat Hamilton eine Dilutoren-Dispensoren-Generation auf den Markt gebracht. Diese Geräteserie wird sowoh als Einzelgeräte mit Kontroller wie auch als OEM-Einbaumodelle gebaut. - De Hamilton Reader HR-7000 gehört zu den präzisesten Mikrotiterplatten-Photometern. Er eignet sich für alle Arte von optischen Dichtemessungen in Mikrotiterplatten. Zusammen mit den welt weit verkauften ML-AT und ML-2200 steilt er ein sehr ökonomisches System ur Blutbanken, Virologielabors und klinische Labors dar. Für die Entwicklung und Produktion von pH-Elektroden setzt Hamilton, neben einem aus Spitzenkräften der Branche zusammengesetzten Forschungs- und Entwicklungsteam, drei Jahrzehnte Erfahrung in Präzisions-Glasbearbeitung ein. Das Ergebnis: dauerhafte und präzise Sensoren zu erstaunlichen Preisen - Der Microlab AT/plus ist Hamiltons neuste Entwick lung. Er ist in der Lage, sehr effizient drei Abbott-Platten zu verarbeiten. RIA-Benutzer profitieren jetzt von einem aussergewöhnlich schnellen Gerät, dessen Potential vor allem darin besteht dass die Anwendungsmöglichkeiten stark erweitert worden sind

Hamilton Medical AG, Via Nova, CH-7403 Rhäzüns, Tel. (081) 372627, Telex 851321 hmedch, Telefax (081) 372689 . Leserdienst 51
Neue Umformer für den Anschluss von Strömungs-/ Durchflussmesswertaufnehmern

Dic Umformer sind für den Anschluss Don Höntzsch-Flügelrad-Messwertaufnehmern (FA) zur Messung der Strömungsgeschwindigkeit oder des Volumenstroms von Luft/Gasen und WasseFlüssigkeiten bestimnt. Sie wandeln die zur Strömungsgeschwindigkeit proportionale Signalírequenz eines Gebers (FA) in einen eingeprägten Strom ode eine eingeprägte Spannung um. Als Anaogsignale stchen nach Wahl 4-20 mA $0-4 \mathrm{~V}, 0-10 \mathrm{~V}$ zur Verfügung. Das Bezugspotential für den Spannungsausgang ist getrennt vom Bezugspotentia für die Versorgung. Die Versorgung kann nach Wahl durch 220 VAC, 24 VAC, 5 VDC, 12 bis 24 VDC erfolgen. In allen Fällen der Fremdspeisung sind die Versorgungsleitungen galvanisch ge- 


\section{Aufträge für Ems-Inventa AG aus Indien}

Das Schweizer Ingenieurunternehmen Ems-Invent AG - eine Gesellschaft der in den Bereichen Polymer Werkstoffe, Feinchemikalien und Engineering tätigen Ems-Gruppe - hat insgesamt 4 Aufträge von den indischen Firmen Haryana Petrochemicals Ltd. und Parasrampuria Synthetics Ltd. erhalten.

Es handelt sich um verschiedene Anlagen zur Herstellung von Polyestergranulat und Polyestergarnen.

Ems-Inventa AG stellt die Technologie, liefert die verfahrensmässig bedingten Maschinen und Einrichtungen, erbringt die Planungs- und Ingenieurarbeiten und stellt das Fachpersonal für die Überwachung der Montage und Inbetriebnahme.

Der gesamte Auftragswert stellt sich auf rund 25 Mio. SFr. Die Anlagen werden in den Staaten Haryana und Rajasthan errichtet. Die Produktionsaufnahme ist innerhalb von 2 Jahren geplant.

\section{Am Montag wurde 'Blau' gemacht:} Indigo, der König der Farbstoffe

I.C. - 'Indigo' - von diesem Wort ging über Jahrhunderte eine ungewöhnliche Faszination aus. Indigo, das war der teure Farbstoff aus fernen Ländern, der den Menschen die Farbe brachte, die sie überall sahen, aber nicht herstellen konnten. Inzwischen jedoch, vor allem dank der Jeans-Welle, ist Indigo ein Massenprodukt, dessen Synthese am 6. Mai dieses Jahres 100 Jahres al wurde.

Schon von jeher hat der Mensch das Bestreben gehabt, scine Unwelt durch Farben zu gestalten. Und während es ihm bereits vor mehr als 10000 Jahren gelang, rote, gelbe, braune und schwarze Farbtöne herzustellen, fehlte ihm lange Zeit das Blau.

In der Natur gibt es diesen Farbstoff - doch musst dessen Synthese erst gefunden werden. In zwei Pflanzenfamilien befindet sich nämlich eine farblose Substanz .. ein Vorprodukt, das heute Indoxyl genann wird. Vom farblosen Vorprodukt zur Farbe gelangte man durch zwei Schritte: zunächst wurde das Pflanzenmaterial zerstossen und dann unter Zusatz von Urin vergoren. Da dieser Prozess in Kübeln stattfand, nannte man die Brühe $K$ üpe. Tränkte man nun gesponnenes oder gewebtes Fasermaterial mit dieser immer noch farblosen Brühe und hängte es dann in die Sonne geschah - durch Oxidation, wie wir heute wissen - das Wunder: ein blauer Farbton entstand.

Bei den indoxylhaltigen Pflanzen handelt es sich zum einen um die zur Familie der Schmetterlingsblütler zählende Indigofera tinctoria, zum anderen um die mi den Kohlgewächsen verwandte Isatis tinctoria au
Deutsch: Färberwaid. Färberwaid wurde in Deutschand im Mittelalter auf grossen Flächen angebaut. Er enthält aber viel weniger farbgebende Substanz als da in den Subtropen wachsende Indigofera tinctoria, das jahrhundertelang in Indien, Indonesien und später auch in den USA landwirtschaftlich angebaut wurde.

\section{Indiga - begehrt und teuer}

Seinen Ursprung hatte das Indigo-Färben im 3. Jahr ' tausend vor unserer Zeitrechnung in - wie der Name erahnen lässt - Indien. Von hier aus trat es ganz langsam seinen Vormarsch nach Westen an und kam erst über den Landweg, dann mit der Entdeckung des Seeweges nach Indien durch Vasco da Gama 1498 nach Europa. Indigo wurde ein begehrtes Handelsproduk und verdrängte immer mehr den heimischen Färberwaid, dessen Blau auch nicht so schön und klar war wie das seines indischen Konkurrenten. Und weil das Blau so selten bzw. so mühsam zu beschaffen war, war es sehr teuer und damit die Farbe der herrschenden Klasse.

Von der Blaufärberei zur industriellen Produktion

Die Färber der damaligen Zeit hatten ein schwere Los. Nach zwölfstündigem, arbeitsintensivem Ansetzen der Küpe und dem Behandeln des Tuches in der Küpe folgte das ebenso lange Aushängen an der Luft Und weil am arbeitsfreien Sonntag das Färbegut länge als sonst - nämlich den ganzen Tag über - in der Küpe blieb, wurde am Montag dann 'Blau gemacht'. Die Gesellen überliessen der Luft den wesentlichen Teil de Arbeit, starteten relativ ruhig in die neue Woche und überlieferten uns so den 'blauen Montag'.

Um die Qualität des Indigo in den Griff und gleichmässigere Färbungen zu bekommen, befasste sich bald die wissenschaftliche Chemie mit dem Indigo. Und da der damals einfachste Weg, eine Substanz zu reinigen deren Verdampfen und anschliessendes Kondensieren war, unterwarf der Apotheker Unverdorben im Jah 1826 den Indigo dieser Prozedur. Der Indigo zersetzte sich dabei zu einer Verbindung, die der Apotheker Anilin nannte, angelehnt an das portugiesische Wort fü Blau. Der Weg in die richtige Richtung für einen ganzen Industriezweig war gewiesen.

\section{Das erste Patent}

Zahlreiche Forscher machten sich an die Arbeit. Es gelang, Anilin aus dem Teer zu gewinnen, der bei der Verkokung yon Steinkohle entstunden war. Erfolge blieben nicht aus. Viele Farbstoffe liessen sich synthetisieren, doch Blau war nicht dabei. Dem Hochschullehrer Adolf von Baeyer gelang es als erstem, das chemische Grundgerüst des Indigo zu entschlüsseln. Nachdem die Molekülstruktur erst einmal bekannt war, liess der nächste Schritt nicht lange auf sich warten. Im März 1880 wurde ihm für den von ihm gefundenen allerdings nicht praktikablen - Syntheseweg ein Paten erteilt. Man hatte 'Blau geleckt'. Weitere neue Synthesewege wurden entdeckt, unter anderem auch von dem Zürcher Professor Karl Heumann Am 6. Mai 1890 liess er sich seinen Weg patentieren, den die chemische Industrie nach weiteren sieben Jahren intensiver Forschung und Entwicklung technisch umsetzen konnte. Der erste künstliche Indigo war auf dem Markt.

Indigo wurde ein Markterfolg. Wichtige Exportlïnder waren China und Amerika, wo der Goldrausch i Kalifornien dem Indigo Aufschwung verlieh. Der ius Bayern nach San Francisco ausgewunderte Levi Strauss fertigte aus mit Indigo gefärbtem Drillich, den er aus dem Textilzentrum Nîmes bezog, die benötigle Arbeitskleidung. Von 'de Nimes' wurde dann in kurzer Zeit der Begriff 'Denim' und aus dem über Genua nach Amerika verschifften Baumwollstoff 'Bleu de Gènes' wurde schncll 'Blue Jeans'.

Jeans das Comeback eines Farbstoffes

Doch mit der Blütezeit des Indigo war es bald vorbci andere blaue Farbstoffe mit besseren Echtheiten wurden erfunden. Und so schwebte lange Zeit das Damoklesschwert der Produktionseinstcllung an cinem hauchdünnen Faden über den Indigofabriken. Abe dann kam in den 60er Jabren das grosse Comeback de Jeans. Die Kleidung, mit dem lichtechten Farbstof Indigo gefärbt, wandelte sich zur Uniform der Nonkonformisten. Die Jeans wurden zum Ausdruck eine veränderten Einstellung, kritisch und unkonventionell, und gehören inzwischen zur legeren Freizcitmode. Dank diesem Comeback wird Indigo heute immer noch grosstechnisch hergestellt.

\section{Schweizer Chemie auf ausreichende Stromversorgung angewiesen}

I.C. - Dank Verfahrensverbesserungen und Sparanstrengungen verbraucht die chemische lndustric in der Schweiz heute gesamthaft nicht mehr Energie als vor zehn Jahren, obwohl im gleichen Zeitraum die Produktion um rund $50 \%$ zunahm. Der Verbrauch an clektrischem Strom nahm allerdings um $27.7 \%$ zu, dies nich zuletzt als Folge der Anstrengungen zum Schutz der Unwelt wie beispielsweise der Betrieb yon Abwasserreinigungsanlagen oder von Elektrofiltern. Weiteres Stromsparen ist nach den jahrelangen Energiesparaktionen in der chemischen Industric kaum mehr möglich. Sic braucht Strom, und zwar aus allen Quellen.

Die chemische Industrie der Schweiz lehnt deshalb die beiden Anti-Atominitiativen (Moratorium und Ausstiegsinitiative) ab, die am 23. September 1990 zur Abstimmung gelangen. Die Schaffung eines Energieartikels in der Bundesverfassung erachtet sic als unnötig ordnungspolitisch verfehlt und wirtschaftlich gefährlich.

\section{Markt: Apparate, Chemikalien und Dienstleistungen}

Mettler-Toledo (Schweiz) AG
neue Adresse ab 1.8.1990:
Mettler-Toledo (Schweiz) AG
Waagen, Wägesysteme und analy-
tische Instrumente, Grabenstrasse 8,
CH-8606 Nänikon-Uster

Produktepalette und Neuheiten von Hamilton Medical

Hamilton Medical entwickelt und vertreibt umfassend die folgenden Geräte: Veolar, das perfekte Hochleistungs-Beatmungsgerät mit allen Extras, die konventionelle und zukünftige Beatmungstechniken erfordern und sicher ausführen. Eine optimale Auswahl an Beatmungsarten für die unterschiedlichsten Anforderungen. - Leonardo, der Patienten Data Manager zur Diagnostik für die Intensivpflegestation IPS. Leonardo er- laubt die Optimierung der Beatmung. Amadeus, der zuverlässige Routine-R spirator für die Intensivstation. Beinhaltet alle konventionellen Beatmungsar ten, Alarme und Monitoring, die für eine sichere, dem Patienten optimal angepasste Beatmung erforderlich sind. Max, der leistungsstarke portable Respirator für Notfall, Intensivmedizin un Transport. Optimale Ventilation selbs unter ungünstigsten Gegebenheiten Einzigartige Kombination von bewäh ter Elcktronik und Präzisionsmechanik. - Neu hat Hamilton eine Dilutoren-Dispensoren-Generation auf den Markt gebracht. Diese Geräteserie wird sowoh als Einzelgeräte mit Kontroller wie auch als OEM-Einbaumodelle gebaut. - De Hamilton Reader HR-7000 gehört zu den präzisesten Mikrotiterplatten-Photometern. Er eignet sich für alle Arte von optischen Dichtemessungen in Mikrotiterplatten. Zusammen mit den welt weit verkauften ML-AT und ML-2200 steilt er ein sehr ökonomisches System ur Blutbanken, Virologielabors und klinische Labors dar. Für die Entwicklung und Produktion von pH-Elektroden setzt Hamilton, neben einem aus Spitzenkräften der Branche zusammengesetzten Forschungs- und Entwicklungsteam, drei Jahrzehnte Erfahrung in Präzisions-Glasbearbeitung ein. Das Ergebnis: dauerhafte und präzise Sensoren zu erstaunlichen Preisen - Der Microlab AT/plus ist Hamiltons neuste Entwick lung. Er ist in der Lage, sehr effizient drei Abbott-Platten zu verarbeiten. RIA-Benutzer profitieren jetzt von einem aussergewöhnlich schnellen Gerät, dessen Potential vor allem darin besteht dass die Anwendungsmöglichkeiten stark erweitert worden sind

Hamilton Medical AG, Via Nova, CH-7403 Rhäzüns, Tel. (081) 372627, Telex 851321 hmedch, Telefax (081) 372689 . Leserdienst 51
Neue Umformer für den Anschluss von Strömungs-/ Durchflussmesswertaufnehmern

Dic Umformer sind für den Anschluss Don Höntzsch-Flügelrad-Messwertaufnehmern (FA) zur Messung der Strömungsgeschwindigkeit oder des Volumenstroms von Luft/Gasen und WasseFlüssigkeiten bestimnt. Sie wandeln die zur Strömungsgeschwindigkeit proportionale Signalírequenz eines Gebers (FA) in einen eingeprägten Strom ode eine eingeprägte Spannung um. Als Anaogsignale stchen nach Wahl 4-20 mA $0-4 \mathrm{~V}, 0-10 \mathrm{~V}$ zur Verfügung. Das Bezugspotential für den Spannungsausgang ist getrennt vom Bezugspotentia für die Versorgung. Die Versorgung kann nach Wahl durch 220 VAC, 24 VAC, 5 VDC, 12 bis 24 VDC erfolgen. In allen Fällen der Fremdspeisung sind die Versorgungsleitungen galvanisch ge- 